\title{
Chemical Synthesis of (+)-Brevianamide a Supports a Diels-Alderase-Free Biosynthesis
}

\author{
Robert Godfrey, Nicholas Green, Gary Nichol, Andrew Lawrence
}

Submitted date: 04/06/2019 Posted date: 05/06/2019

Licence: CC BY-NC-ND 4.0

Citation information: Godfrey, Robert; Green, Nicholas; Nichol, Gary; Lawrence, Andrew (2019): Chemical Synthesis of (+)-Brevianamide a Supports a Diels-Alderase-Free Biosynthesis. ChemRxiv. Preprint.

The fungal-derived bicyclo[2.2.2]diazaoctane alkaloids are of significant interest to the scientific community for their potent and varied biological activities. Within this large and diverse family of natural products the insecticidal metabolite (+)-brevianamide $A$ is particularly noteworthy for its synthetic intractability and inexplicable biogenesis. Despite five decades of research, this alkaloid has never succumbed to chemical synthesis. It has been suggested that a proposed Diels-Alder reaction in the biosynthesis of (+)-brevianamide A requires a Diels-Alderase enzyme. We herein report the first chemical synthesis of (+)-brevianamide $A$ (7 steps, $8.0 \%$ overall yield, $750 \mathrm{mg}$ scale), which provides compelling evidence in support of a Diels-Alderase-free biosynthesis; a significant departure from the established biosynthesis of related alkaloids.

File list (2)

Brevianamide A - Manuscript.pdf (2.01 MiB) view on ChemRxiv • download file 
Chemical synthesis of (+)-brevianamide A supports a Diels-Alderase-free biosynthesis.

Robert C. Godfrey, Nicholas J. Green, Gary S. Nichol and Andrew L. Lawrence*

EaStCHEM School of Chemistry, University of Edinburgh, Joseph Black Building, David Brewster Road, Edinburgh, EH9 3FJ, UK. E-mail: a.lawrence@ed.ac.uk
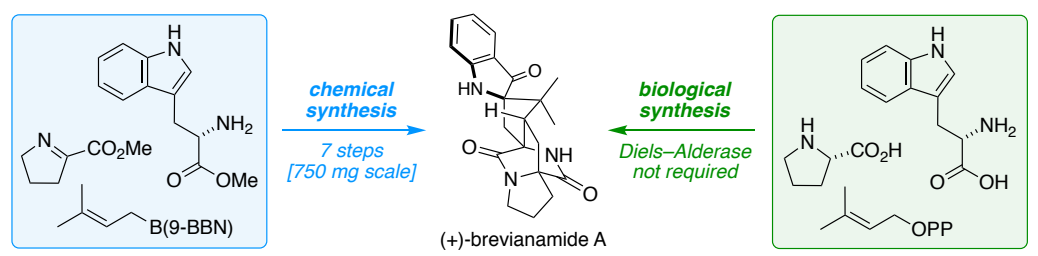

The fungal-derived bicyclo[2.2.2] diazaoctane alkaloids are of significant interest to the scientific community for their potent and varied biological activities. Within this large and diverse family of natural products the insecticidal metabolite $(+)$-brevianamide $A$ is particularly noteworthy for its synthetic intractability and inexplicable biogenesis. Despite five decades of research, this alkaloid has never succumbed to chemical synthesis. It has been suggested that a proposed Diels-Alder reaction in the biosynthesis of (+)-brevianamide A requires a Diels-Alderase enzyme. We herein report the first chemical synthesis of (+)-brevianamide A (7 steps, $8.0 \%$ overall yield, $750 \mathrm{mg}$ scale), which provides compelling evidence in support of a Diels-Alderase-free biosynthesis; a significant departure from the established biosynthesis of related alkaloids.

The bicyclo[2.2.2]diazaoctane alkaloids are a vast group of natural products isolated from various marine and terrestrial fungi. ${ }^{1,2}$ They are of significant interest to chemists and biologists alike due to their important biological activities, diverse biosynthetic origins, and synthetically daunting structures. There are two distinct families of bicyclo[2.2.2]diazaoctane alkaloids, the monooxopiperazine-type structures, which include the anthelmintic paraherquamides, ${ }^{3-5}$ calmodulin-inhibiting malbrancheamides, ${ }^{6}$ and neuroprotective 
chrysogenamides ${ }^{7}$ and the dioxopiperazine-type structures, which include the anti-tumoral stephacidins,${ }^{8}$ cytotoxic notoamides,${ }^{9}$ and insecticidal brevianamides (Figure 1). ${ }^{10,11}$

Brevianamides A (1) and B (2) were originally isolated by Birch and Wright in 1969 from the fungus Penicillium brevicompactum, ${ }^{10}$ and were the first known bicyclo[2.2.2] diazaoctane alkaloids (Figure 1)., Brevianamide A (1) exhibits potent antifeedant activity against the larvae of the insect pests Spodoptera frugiperda (fall armyworm) and Heliothis virescens (tobacco budworm), ${ }^{11}$ and is the major isolated diastereomer (ratio of A to B $\gtrsim 90: 10$ ). ${ }^{12,13}$ In 1970, Porter and Sammes proposed that the bicyclo[2.2.2] diazaoctane cores of brevianamides A (1) and B (2) could be biosynthesised through an intramolecular hetero-Diels-Alder cycloaddition (Figure 1), ${ }^{14}$ a proposal which has been extended to encompass all bicyclo[2.2.2]diazaoctane alkaloids. ${ }^{1,2}$ Recently, DielsAlderase enzymes have been identified in the biosynthetic gene clusters responsible for the malbrancheamide and paraherquamide monooxopiperazine-type alkaloids. ${ }^{15}$ However, no Diels-Alderase enzyme has yet been identified for the brevianamides, nor any other dioxopiperazine-type alkaloid. ${ }^{16}$ Furthermore, despite five decades of research, no chemical synthesis of brevianamide A (1) has been achieved, yet several syntheses of the minor diastereomer, brevianamide B (2), have been reported (see SI for a full summary). ${ }^{17-25}$ Despite the similar structures of the two natural products, none of these strategies have been successfully applied to the synthesis of brevianamide A (1) due to insurmountable issues of stereoselectivity.
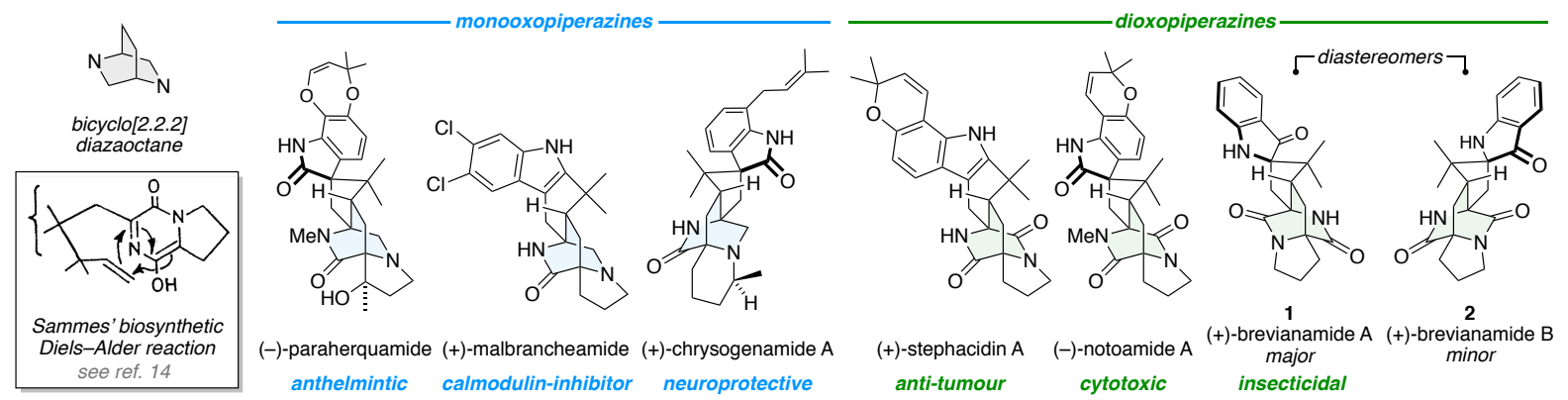

Fig 1: Representative bicyclo[2.2.2]diazaoctane alkaloids (bicyclo[2.2.2]diazaoctane cores highlighted). 
Following decades of detailed chemical and biochemical studies, the biosynthetic origins of (+)-brevianamide A (1) and B (2) still remain largely unknown. We herein report a new biosynthetic hypothesis, which builds upon the pioneering work of several research groups. $^{1,2}$ To appreciate the origins of our new biosynthetic proposal it is important to first outline key details of the elegant work of Williams and co-workers. ${ }^{19-22}$ Their biomimetic synthetic studies have mainly focused upon an early proposed pathway (Figure 2, Pathway 1), which was informed by the seminal work of Birch and Sammes. ${ }^{14,27-29}$ The pathway begins with a stereoablative oxidation of (+)-deoxybrevianamide E (3) to give achiral azadiene 4, which then undergoes enantioselective Diels-Alder cycloaddition to give a scalemic mixture of bicyclo[2.2.2]diazaoctane enantiomers, 5 and ent-5. Both enantiomers then undergo $(R)$-selective indole oxidation and a [1,2]-alkyl shift, so that the major enantiomer (5) gives brevianamide A (1) and the minor enantiomer (ent-5) gives brevianamide B (2). Williams' synthetic studies, however, revealed that this proposed DielsAlder reaction (4 to 5/ent-5) actually produces an unwanted diastereomer as the major product. $^{22}$ Therefore, a Diels-Alderase enzyme would be required to reverse this inherent diastereoselectivity and yet provide only modest enantioselectivity. Further evidence against this proposed pathway was provided through biosynthetic feeding experiments with isotopically labelled $\mathbf{5}$, which failed to show any significant incorporation into (+)-brevianamide A (1). ${ }^{30}$ Williams, therefore, proposed an alternative biosynthetic pathway (Figure 2, Pathway 2), which begins with a diastereoselective indole oxidation of (+)-deoxybrevianamide E (3) to give hydroxyindolenine 6. ${ }^{30,31}$ A stereospecific $[1,2]$-alkyl shift then gives indoxyl 7, which undergoes a diketopiperazine oxidation and Diels-Alder cycloaddition to give brevianamides A (1) and B (2). ${ }^{30,31}$ Williams and co-workers reasoned that "[t]he preponderance of $\mathbf{1}$ over $\mathbf{2}$ would be due either to the relative activities of two different [Diels-Alderase] enzymes or the affinity of a single enzyme active site for the individual conformers". ${ }^{30}$ All efforts to substantiate this second-generation pathway in vitro, 
however, have failed, largely due to the instability of indoxyl 7. ${ }^{1}$ Furthermore, hydroxyindolenine 6 is likely to undergo rapid 5-exo-trig cyclisation to give the known shunt-metabolite brevianamide E (8), ${ }^{10,27}$ which would represent a biosynthetic dead-end. ${ }^{30}$

Our new biosynthetic hypothesis for (+)-brevianamide A (1) involves an alternative biosynthetic precursor, (+)-dehydrodeoxybrevianamide E (9), which is a known natural product isolated from various Penicillium and Aspergillus species (Figure 2, Pathway 3). ${ }^{32-34}$ Crucially, the diketopiperazine ring in (+)-dehydrodeoxybrevianamide E (9) is already at the oxidation level required for a later Diels-Alder reaction. Our pathway involves a point-topoint chirality transfer via a sequential diastereoselective indole oxidation, stereospecific [1,2]-alkyl shift, and tautomerisation, to give enantiopure azadiene 10. Diels-Alder cycloaddition of azadiene 10 would then give brevianamides A (1) and B (2). ${ }^{30,31}$ Although our proposed intermediate $\mathbf{1 1}$ is likely to undergo reversible 5-exo-trig cyclisation to give pentacycle 12, this is not a known natural product and is therefore unlikely to represent a biosynthetic dead-end (cf. brevianamide E (8) in Pathway 2). We herein report our synthetic investigation into the chemical feasibility of this new biosynthetic proposal, which has resulted in the first total synthesis of this infamous natural product. 


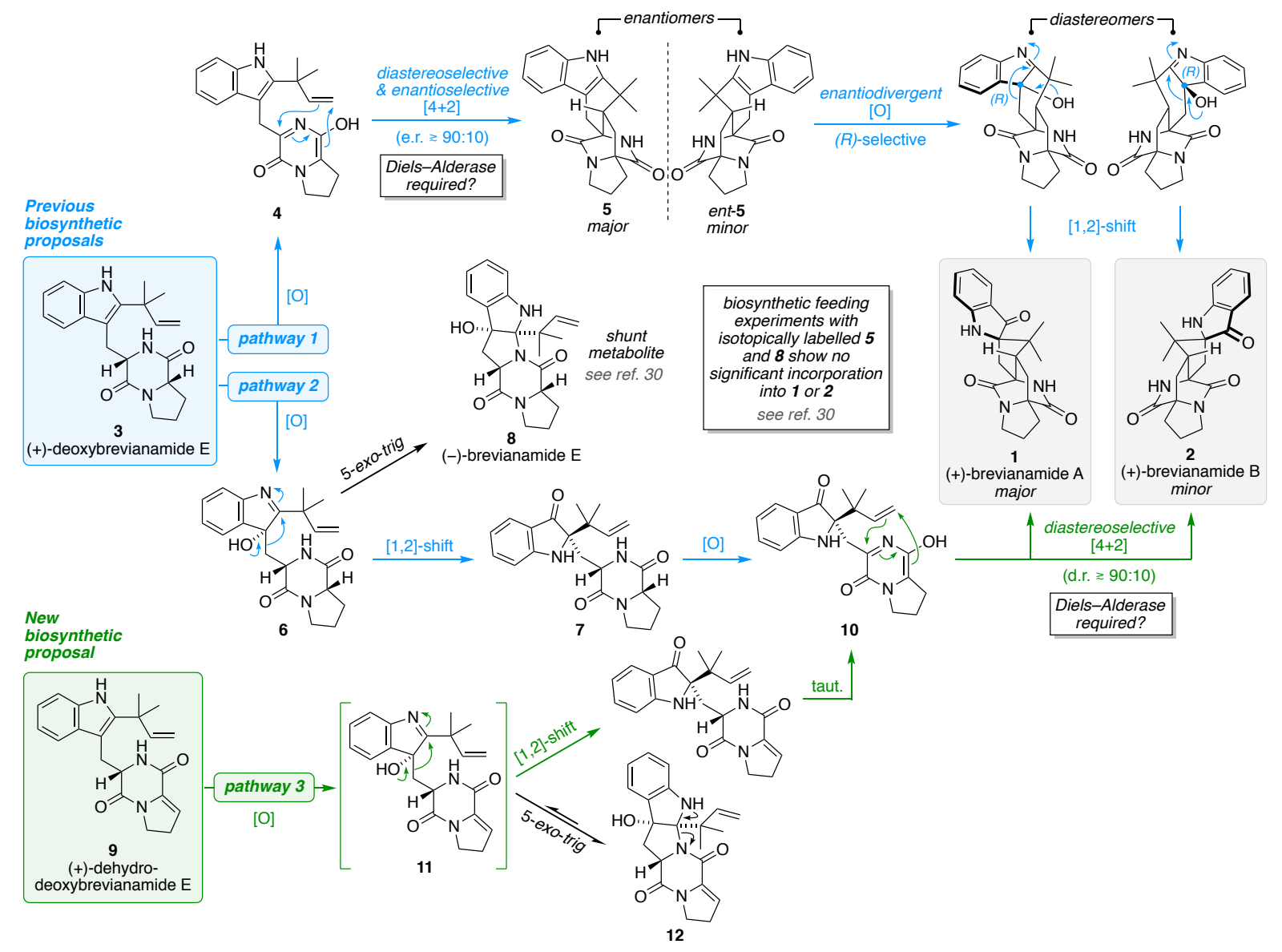

Fig 2: Biosynthetic proposals for brevianamides A and B.

\section{Results and Discussion}

The synthesis of our proposed biosynthetic precursor, $(+)$-dehydrodeoxybrevianamide E (9), commenced with phthaloyl protection of commercially available L-tryptophan methyl ester 13 (Figure 3). ${ }^{35}$ The crude product from this reaction, ester 14, was subjected directly to Danishefsky's reverse prenylation conditions using $B$-prenyl-9-BBN to give more than 20 grams of intermediate $15 .{ }^{36}$ Hydrolysis of methyl ester 15 was accompanied by ring opening of the phthaloyl group to give diacid 16, which presumably explains why a protecting group switch from phthaloyl to tert-butyloxycarbonyl or trityl has been undertaken in related synthetic endeavours. ${ }^{36,37}$ To avoid these extraneous two steps (deprotection/re-protection) we developed a quantitative $\mathrm{S}_{\mathrm{N}} 2$-type demethylation of methyl ester 15 using $\mathrm{LiCl}$ in $\mathrm{DMF} .{ }^{38}$ Lithium carboxylate 17 was then subjected to a one-pot acyl chloride formation and imine acylation reaction with dehydroproline $\mathbf{1 8}$ to give $N$-acyl 
enamine 19. ${ }^{39}$ Attempts to deprotect the primary amine in $\mathbf{1 9}$ using conventional phthaloyl deprotection reagents, such as hydrazine, resulted in unwanted cleavage of the amide bond. Our search for less nucleophilic reagents eventually led us to ammonia in methanol, which not only deprotected the primary amine but also resulted in spontaneous cyclisation to give more than eight grams of (+)-dehydrodeoxybrevianamide E (9) in $49 \%$ yield over the three steps from methyl ester $\mathbf{1 5}$. Thus, by developing a new imine acylation reaction and avoiding unnecessary protecting group manipulations, the shortest total synthesis of (+)-dehydrodeoxybrevianamide E (9) has been achieved, proceeding in a longest linear sequence of 5 steps, 34\% overall yield, and requiring just two chromatographic purifications (cf. previous synthesis: 12 steps, $8 \%$ overall yield). ${ }^{22}$

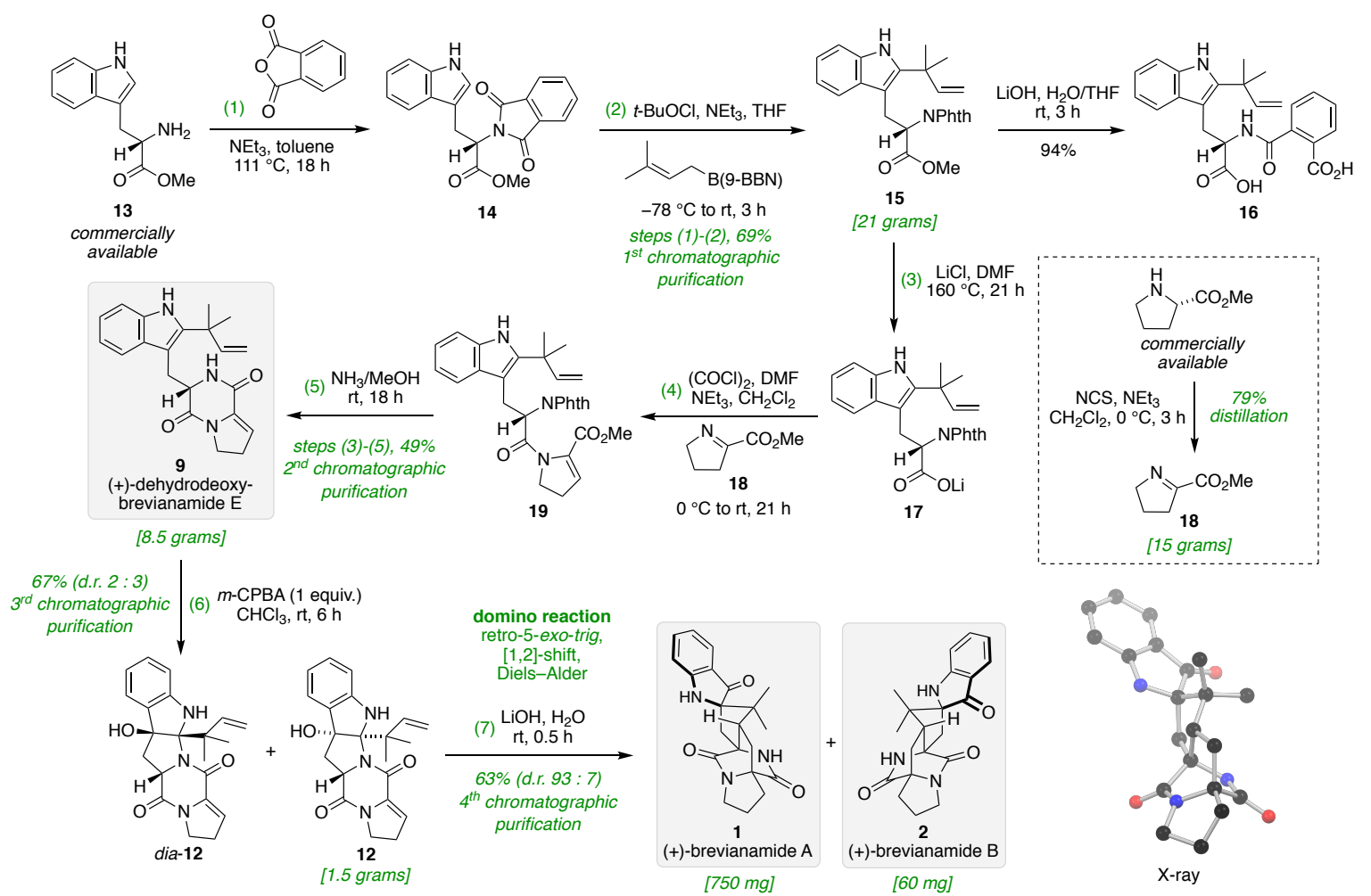

Fig 3: Total synthesis of (+)-brevianamides A and B. 9-BBN, 9-borabicyclo(3.3.1)nonane; Phth, phthaloyl; DMF, dimethylformamide; NCS, $N$-chlorosuccinimide; $m$-CPBA, meta-chloroperoxybenzoic acid.

Oxidation of (+)-dehydrodeoxybrevianamide E (9) with $m$-CPBA gave our proposed dehydro-analogue of brevianamide E, 12, and a minor diastereomer dia-12 in 67\% combined yield (d.r. 12:dia-12, 3:2). Exposure of intermediate 12 to $\mathrm{LiOH}$ in water at ambient 
temperature successfully gave (+)-brevianamide A (1) and (+)-brevianamide B (2) in a combined $63 \%$ yield, presumably via the domino retro-5-exo-trig/[1,2]-alkyl shift/DielsAlder reaction sequence as outlined in Figure 2 (Pathway 3). Following purification by column chromatography, which is only the fourth purification in the entire seven-step synthesis, 750 milligrams of (+)-brevianamide A (1) and 60 milligrams of (+)-brevianamide B (2) were isolated. ${ }^{40}$ The unnatural (-)-enantiomers of brevianamides A (1) and B (2) could also be accessed by subjecting the minor diastereomer, dia-12, to the same reaction conditions (see SI for full details). The biomimetic Diels-Alder reaction produces (+)-brevianamide A (1) and (+)-brevianamide B (2) in a 93:7 diastereomeric ratio (Figure 3), which closely matches the ratio observed when they are isolated from Penicillium brevicompactum (see SI for further details). ${ }^{12}$ This result demonstrates that Diels-Alderase catalysis is not necessary to achieve stereocontrol in the biosynthesis of the brevianamides. ${ }^{30}$ Indeed, if the biosynthetic Diels-Alder reaction is enzyme catalysed, the putative DielsAlderase enzyme must lower the energy barrier without exerting any stereocontrol (within experimental error). ${ }^{41-43}$ To the best of our knowledge, this would represent an unprecedented discovery for Diels-Alderase enzymes. ${ }^{44-46}$ Thus, we suggest that this Diels-Alder reaction is instead a spontaneous process exploited by Nature without direct enzyme participation. This is in stark contrast to the requirement of Diels-Alderase enzymes in the stereoselective biogenesis of the closely related malbrancheamide and paraherquamide alkaloids. ${ }^{15}$

In summary, 50 years after its discovery by Birch, ${ }^{10}$ the first chemical synthesis of brevianamide A (1) has finally been achieved. This synthesis provides valuable insights into a previously enigmatic biosynthetic pathway and demonstrates the utility of the biomimetic approach in the synthesis of complex natural products. 


\section{Acknowledgements}

This work was supported by an EPSRC First Grant (EP/N029542/1) and a Marie Curie Career Integration Grant (631132, POSIN). We would like to thank Mr Tony Herlt for assistance and advice regarding chromatography, and acknowledge SIRCAMS at the University of Edinburgh for mass spectrometry.

\section{Author Contributions}

R.C.G., N.J.G. and A.L.L. conceived, designed and carried out the synthetic experiments. G.S.N. performed the crystallographic studies. All authors discussed and co-wrote the manuscript.

\section{References:}

1. Finefield, J. M., Frisvad, J. C., Sherman, D. H. \& Williams, R. M. Fungal origins of the bicyclo[2.2.2]diazaoctane ring system of prenylated indole alkaloids. J. Nat. Prod. 75, 812-833 (2012).

2. Klas, K. R., et al. Structural and stereochemical diversity in prenylated indole alkaloids containing the bicyclo[2.2.2]diazaoctane ring system from marine and terrestrial fungi. Nat. Prod. Rep. 35, 532-558 (2018).

3. Robertson, A. P., et al. Paraherquamide and 2-deoxy-paraherquamide distinguish cholinergic receptor subtypes in Ascaris muscle. J. Pharmacol. Exp. Ther. 303, 853860 (2002).

4. Little, P. R. et al. Efficacy of a combined oral formulation of derquantel-abamectin against the adult and larval stages of nematodes in sheep, including anthelminticresistant strains. Vet. Parasitol. 181, 180-193 (2011).

5. Buxton, S. K. et al. Investigation of acetylcholine receptor diversity in a nematode parasite leads to characterization of tribendimidine- and derquantel-sensitive nAChRs. PLoS Pathog. 10, e1003870 (2014). 
6. Martínez-Luisa, S., et al. Malbrancheamide, a new calmodulin inhibitor from the fungus Malbranchea aurantiaca. Tetrahedron 62, 1817-1822 (2006).

7. Lin, Z., et al. Chrysogenamide a from an endophytic fungus associated with Cistanche deserticola and its neuroprotective effect on SH-SY5Y cells. J. Antibiot. 61, 81-85 (2008).

8. Qian-Cutrone, J., et al. Stephacidin A and B: two structurally novel, selective inhibitors of the testosterone-dependent prostate LNCaP cells. J. Am. Chem. Soc. 124, 14556-14557 (2002).

9. Kato, H., et al. Notoamides A-D: Prenylated indole alkaloids isolated from a marinederived fungus, Aspergillus sp. Angew. Chem. Int. Ed. 46, 2254-2256 (2007).

10. Birch, A. J. \& Wright, J. J. The brevianamides: a new class of fungal alkaloid. $J$. Chem. Soc. D, Chem. Commun. 644-645 (1969).

11. Paterson, R. R. M., Simmonds, M. J. S., Kemmelmeier, C. \& Blaney, W. M. Effects of brevianamide A, its photolysis product brevianamide D, and ochratoxin A from two Penicillium strains on the insect pests Spodoptera frugiperda and Heliothis virescens. Mycol. Res. 94, 538-542 (1990).

12. Bird, B. A. \& Campbell, I. M. Brevianamides A and B are formed only after conidiation has begun in solid cultures of Penicillium brevicompactum. Appl. Environ. Microbiol. 42, 521-525 (1981).

13. Bird, B. A., Remaley, A. T. \& Campbell, I. M. Disposition of mycophenolic acid, brevianamide A, asperphenamate, and ergosterol in solid cultures of Penicillium brevicompactum. Appl. Environ. Microbiol. 43, 345-348 (1982).

14. Porter, A. E. A. \& Sammes, P. G. A Diels-Alder reaction of possible biosynthetic importance. J. Chem. Soc. D 1103-1103 (1970). 
15. Dan, Q., et al. Fungal indole alkaloid biogenesis through evolution of a bifunctional reductase/Diels-Alderase. Preprint at https://doi.org/10.26434/chemrxiv.7505486.v1 (2018).

16. Li, S., et al. Comparative analysis of the biosynthetic systems for fungal bicyclo[2.2.2]diazaoctane indole alkaloids: the $(+) /(-)$-notoamide, paraherquamide and malbrancheamide pathways. Med. Chem. Commun. 3, 987-996 (2012).

17. Williams, R. M., Glinka, T. \& Kwast, E. Facial selectivity of the intramolecular $\mathrm{S}_{\mathrm{N}} 2^{\prime}$ cyclization: stereocontrolled total synthesis of brevianamide B. J. Am. Chem. Soc. 110, 5927-5929 (1988).

18. Williams, R. M., Glinka, T., Kwast, E., Coffman, H. \& Stille, J. K. Asymmetric, stereocontrolled total synthesis of (-)-brevianamide B. J. Am. Chem. Soc. 112, 808$821(1990)$.

19. Williams, R. M., Sanz-Cervera, J. F., Sancenón, F., Marco, J. A. \& Halligan, K. Biomimetic Diels-Alder cyclizations for the construction of the brevianamide, paraherquamide sclerotamide, and VM55599 ring systems. J. Am. Chem. Soc. 120, 1090-1091 (1998).

20. Williams, R. M., Sanz-Cervera, J. F., Sancenón, F., Marco, J. A. \& Halligan, K. Biomimetic Diels-Alder cyclizations for the construction of the brevianamide, paraherquamide, sclerotamide, asperparaline and VM55599 ring systems. Bioorg. Med. Chem. 6, 1233-1241 (1998).

21. Adams, L. A., Valente, M. W. N. \& Williams, R. M. A concise synthesis of d,1brevianamide B via a biomimetically-inspired IMDA construction. Tetrahedron $\mathbf{6 2}$, 5195-5200 (2006).

22. Greshock, T. J. \& Williams, R. M. Improved biomimetic total synthesis of d,1stephacidin A. Org. Lett. 9, 4255-4258 (2007). 
23. Frebault, F. C. \& Simpkins, N. S. A cationic cyclisation route to prenylated indole alkaloids: synthesis of malbrancheamide $\mathrm{B}$ and brevianamide $\mathrm{B}$, and progress towards stephacidin A. Tetrahedron 66, 6585-6596 (2010).

24. Robins, J. G., Kim, K. J., Chinn, A. J., Woo, J. S. \& Scheerer, J. R. Intermolecular Diels-Alder Cycloaddition for the Construction of Bicyclo[2.2.2]diazaoctane Structures: Formal Synthesis of Brevianamide B and Premalbrancheamide. J. Org. Chem. 81, 2293-2301 (2016).

25. Perkins, J. C., Wang, X., Pike, R. D. \& Scheerer, J. R. Further investigation of the intermolecular Diels-Alder cycloaddition for the synthesis of bicyclo[2.2.2]diazaoctane alkaloids. J. Org. Chem. 82, 13656-13662 (2017).

26. Williams, R. M., Kwast, E., Coffman, H. \& Glinka, T. Remarkable, enantiodivergent biogenesis of brevianamide A and B. J. Am. Chem. Soc. 111, 3064-3065 (1989).

27. Birch, A. J. \& Wright, J. J. Studies in relation to biosynthesis-XLII: The structural elucidation and some aspects of the biosynthesis of the brevianamides-A and -E. Tetrahedron 26, 2329-2344 (1970).

28. Birch, A. J. \& Russell, R. A. Studies in relation to biosynthesis-XLIV: Structural elucidations of brevianamides-B, -C, -D and -F. Tetrahedron 28, 2999-3008 (1972).

29. Baldas, J., Birch, A. J. \& Russell, R. A. Studies in relation to biosynthesis. Part XLVI. Incorporation of cyclo-L-tryptophyl-L-proline into brevianamide A. J. Chem. Soc., Perkin Trans. 1, 50-52 (1974).

30. Sanz-Cervera, J. F., Glinka, T. \& Williams, R. M. Biosynthesis of brevianamides A and B: In search of the biosynthetic Diels-Alder construction. Tetrahedron 49, 8471-8482 (1993).

31. Domingo, L. R., Sanz-Cervera, J. F., Williams, R. M., Picher, M. T. \& Marco, J. A. Biosynthesis of the brevianamides. An ab initio study of the biosynthetic intramolecular Diels-Alder cycloaddition. J. Org. Chem. 62, 1662-1667 (1997). 
32. Steyn, P. S. The structures of five diketopiperazines from Aspergillus ustus. Tetrahedron 29, 107-120 (1973).

33. Scott, P. M., Kennedy, B. P. C., Harwig, J. \& Chen, Y-K. Formation of diketopiperazines by Penicillium italicum isolated from oranges. Appl. Microbiol. 28, 892-894 (1974).

34. Kaur, A., Raja, H. A., Deep, G., Agarwal, R. \& Oberlies, N. H. Pannorin B, a new naphthopyrone from an endophytic fungal isolate of Penicillium sp. Magn. Reson. Chem. 54, 164-167 (2016).

35. Liu, H., Pattabiraman, V. R. \& Vederas, J. C., Stereoselective syntheses of 4-oxa diaminopimelic acid and its protected derivatives via aziridine ring opening. Org. Lett. 9, 4211-4214 (2007).

36. Schkeryantz, J. M., Woo, J. C. G., Siliphaivanh, P., Depew, K. M. \& Danishefsky, S. J. Total synthesis of gypsetin, deoxybrevianamide E, brevianamide E, and tryprostatin B: Novel constructions of 2,3-disubstituted indoles. J. Am. Chem. Soc. 121, 11964 11975 (1999).

37. Zhao, L., May, J. P., Huang, J. \& Perrin D. M. Stereoselective synthesis of brevianamide E. Org. Lett. 14, 90-93 (2012).

38. Fisher, J. W. \& Trinkle, K. L. Iodide dealkylation of benzyl, PMB, PNB, and $t$-Butyl $N$-acyl amino acid esters via lithium ion coordination. Tetrahedron Lett. 35, 25052508 (1994).

39. Huy, P., Neudörfl, J.-M. \& Schmalz, H.-G. A practical synthesis of trans-3substituted proline derivatives through 1,4-addition. Org. Lett. 13, 216-219 (2011).

40. CCDC 1918446 (1) contains the supplementary crystallographic data for this paper. The data is provided free of charge by the Cambridge Crystallographic Data Centre.

41. Winkler, N. W. \& Markovitz, A. Guanosine diphosphate-4-keto-D-rhamnose reductase a non-stereoselective enzyme. J. Biol. Chem. 246, 5868-5876 (1971). 
42. Motosugi, K., Esaki, N. \& Soda, K. Purification and properties of a new enzyme, DL2-haloacid dehydrogenase, from Pseudomonas sp. J. Bacteriol. 150, 522-527 (1982).

43. Ding, W., Li, Y. \& Zhang, Q. Substrate-controlled stereochemistry in natural product biosynthesis. ACS Chem. Biol. 10, 1590-1598 (2015).

44. Klas, K., Tsukamoto, S., Sherman, D. H. \& Williams, R. M. Natural Diels-Alderases: Elusive and irresistable. J. Org. Chem. 80, 11672-11685 (2015).

45. Minami, A. \& Oikawa, H. Recent advances of Diels-Alderases involved in natural product biosynthesis J. Antibiot. 69, 500-506 (2016).

46. Jamieson, C. S., Ohashi, M., Liu, F., Tang, Y. \& Houk, K. N. The expanding world of biosynthetic pericyclases: cooperation of experiment and theory for discovery. Nat. Prod. Rep. 36, 698-713 (2019). 


\section{SUPPORTING INFORMATION}

Chemical synthesis of (+)-brevianamide A supports a Diels-Alderase-free biosynthesis.

Robert C. Godfrey, Nicholas J. Green, Gary S. Nichol and Andrew L. Lawrence*

EaStCHEM School of Chemistry, University of Edinburgh, Joseph Black Building, David Brewster Road, Edinburgh, UK, EH9 3FJ. E-mail: a.lawrence@ed.ac.uk 


\section{Supporting Information - Table of Contents}

1. Overview of previous total and formal syntheses of brevianamide B............................... 4

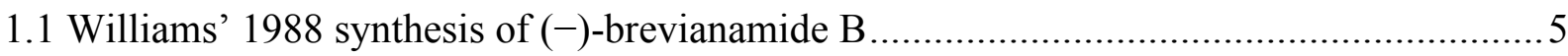

1.2 Williams' 1998 synthesis of $( \pm)$-brevianamide B .................................................... 6

1.3 Williams' 2006 synthesis of $( \pm)$-brevianamide B ................................................ 7

1.4 Williams' 2007 synthesis of $( \pm)$-brevianamide B \& $( \pm)$-dehydrodeoxybreviaminde E .......8

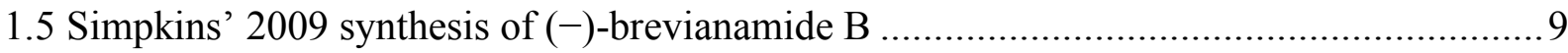

1.6 Scheerer's 2016 formal synthesis of $( \pm)$-brevianamide B ............................................. 10

1.7 Scheerer's 2017 formal synthesis of $( \pm)$-brevianamide B............................................ 11

2. Quantitative analysis of brevianamide A and B in Penicillium brevicompactum .................. 12

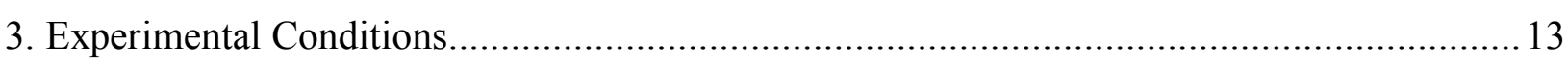

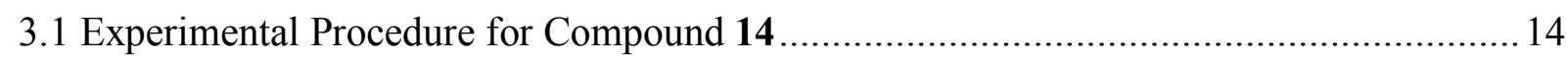

3.2 Experimental Procedure for Compound 15 ............................................................ 15

3.3 Experimental Procedure for Compound 16 ....................................................... 16

3.4 Experimental Procedure for Compound 17 ................................................................. 17

3.5 Experimental Procedure for Compound 19 .......................................................... 18

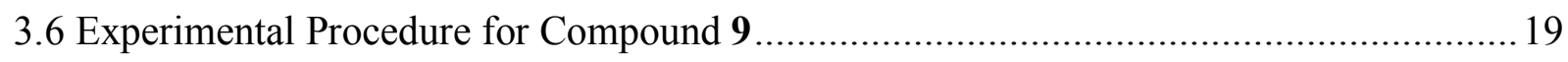

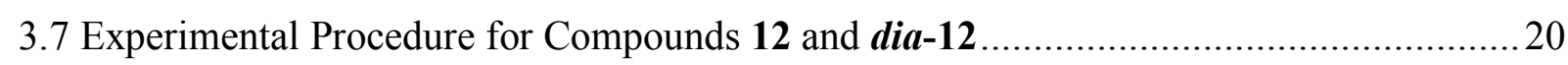

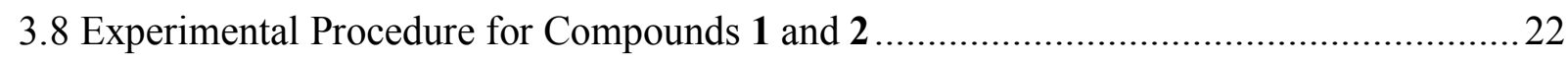

3.9 Experimental Procedure for Compounds ent-1 and ent-2 ...........................................25

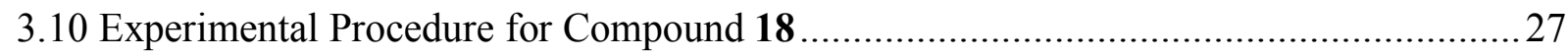

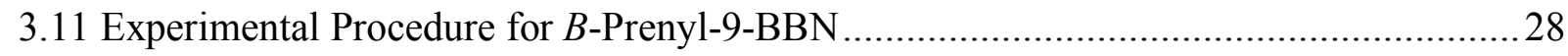

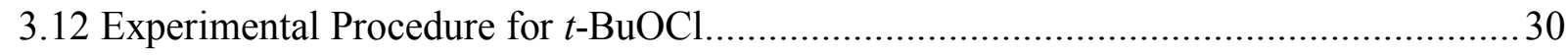

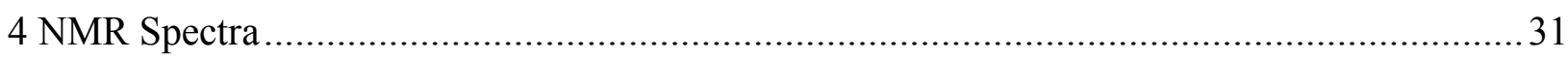

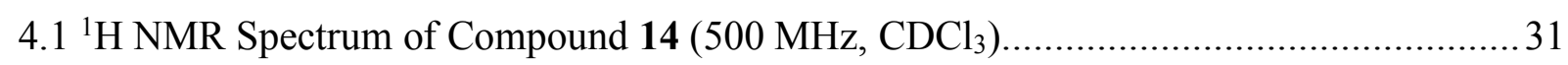

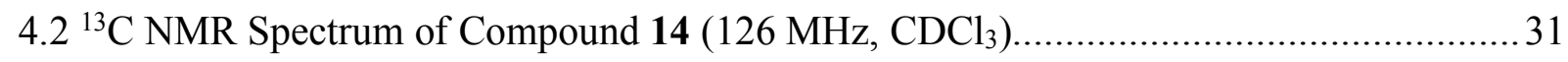

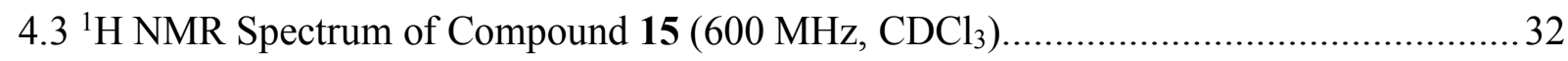

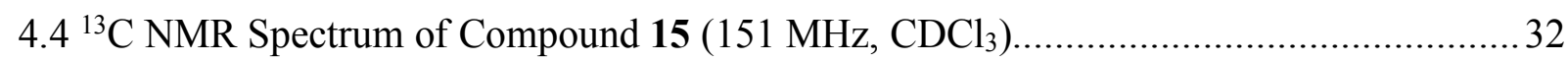

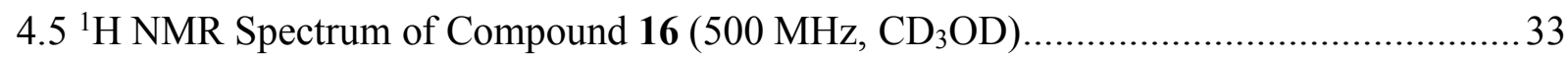

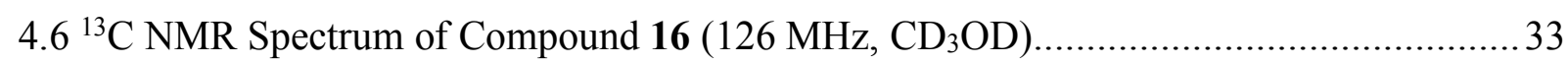

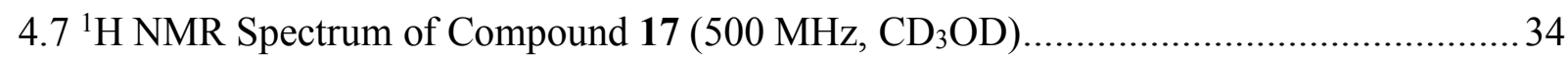

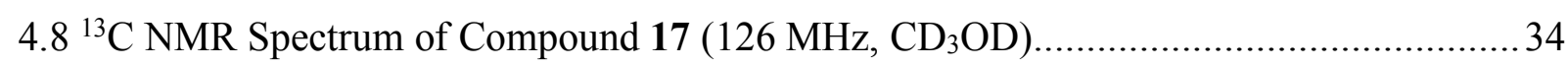

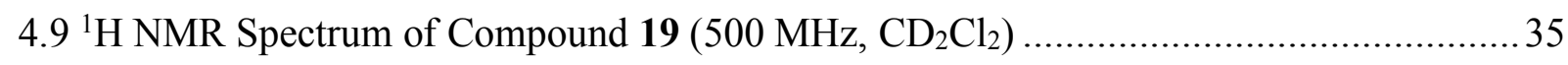

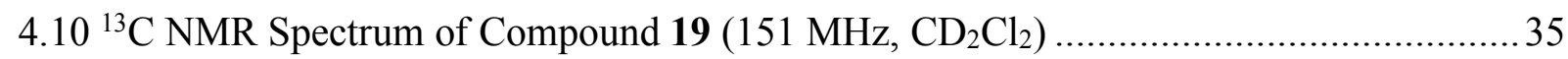

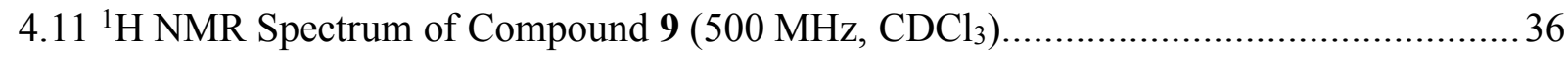

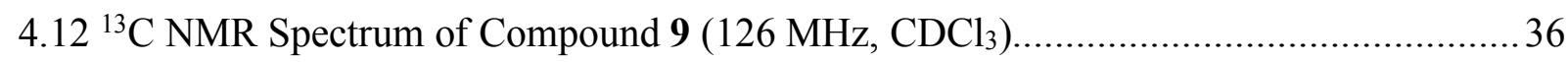

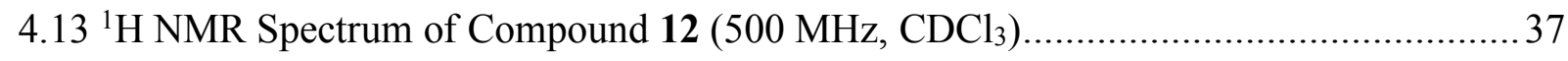

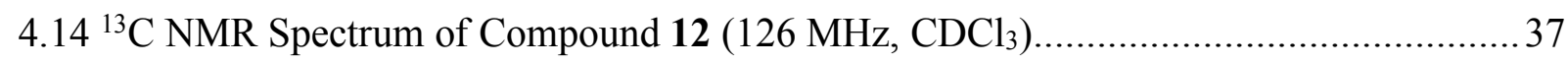

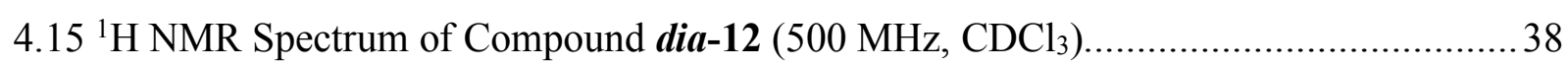

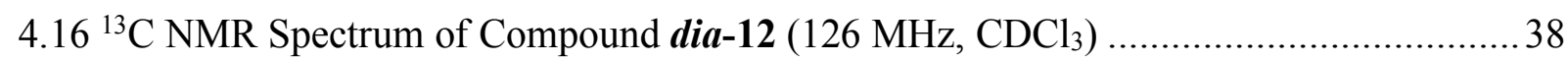


$4.17{ }^{1} \mathrm{H}$ NMR Spectrum of Brevianamide A (1) (500 MHz, $\left.\mathrm{CDCl}_{3}, 3.0 \mathrm{mg} / \mathrm{mL}\right)$ 39

$4.18{ }^{13} \mathrm{C}$ NMR Spectrum of Brevianamide A (1) (126 MHz, $\left.\mathrm{CDCl}_{3}, \sim 3.0 \mathrm{mg} / \mathrm{mL}\right)$. 39

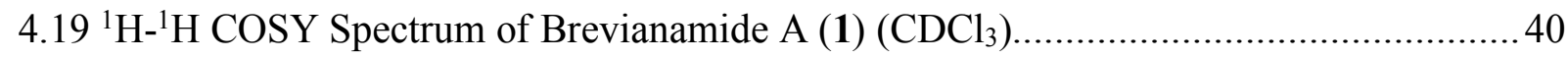

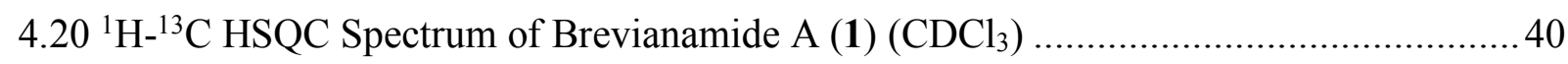

$4.21{ }^{1} \mathrm{H}_{-}{ }^{13} \mathrm{C}$ HMBC Spectrum of Brevianamide A (1) $\left(\mathrm{CDCl}_{3}\right)$.......................................... 41

$4.22{ }^{1} \mathrm{H}-{ }^{1} \mathrm{H}$ NOESY Spectrum of Brevianamide A (1) $\left(\mathrm{CDCl}_{3}\right)$........................................ 41

$4.23{ }^{1} \mathrm{H}$ NMR Spectra of Brevianamide A (1) (500 MHz, $\mathrm{CDCl}_{3}$ - conc. dependence) .......... 42

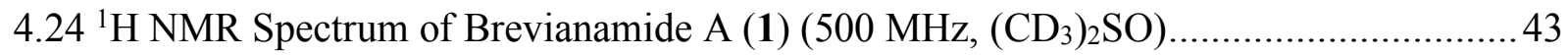

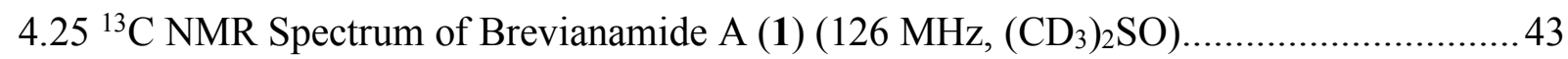

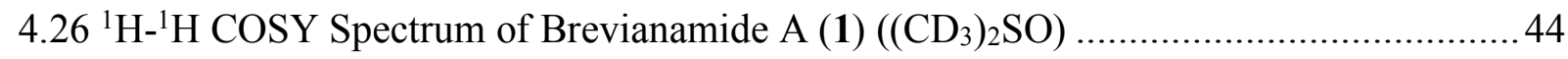

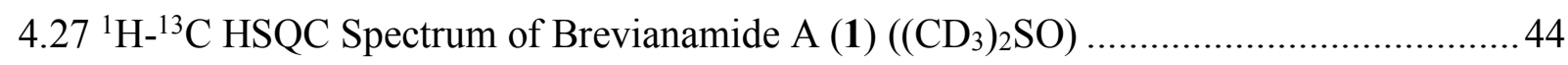

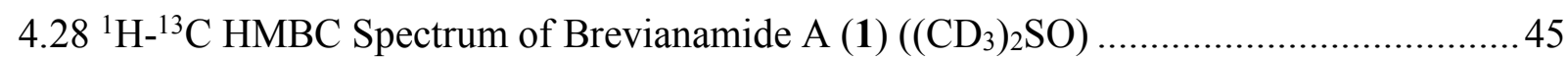

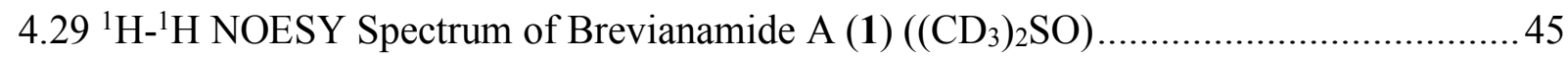

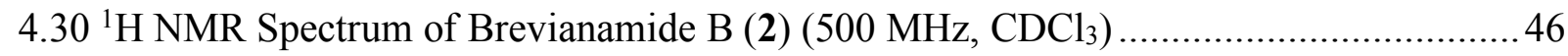

$4.31{ }^{13} \mathrm{C}$ NMR Spectrum of Brevianamide B (2) $\left(126 \mathrm{MHz}, \mathrm{CDCl}_{3}\right)$...................................46

$4.32{ }^{1} \mathrm{H}$ NMR Spectrum of Brevianamide B (2) $\left(500 \mathrm{MHz},\left(\mathrm{CD}_{3}\right)_{2} \mathrm{SO}\right)$............................... 47

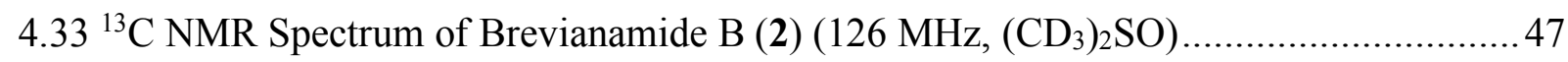

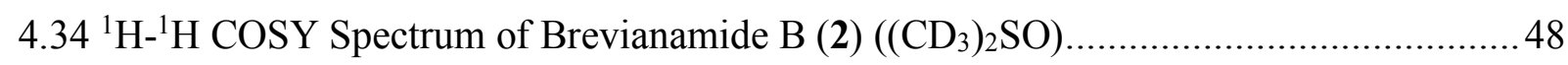

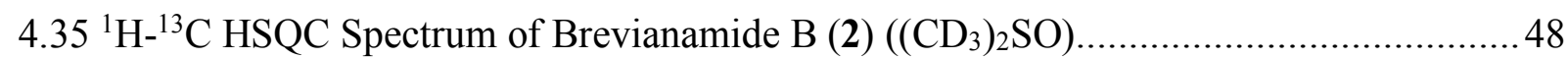

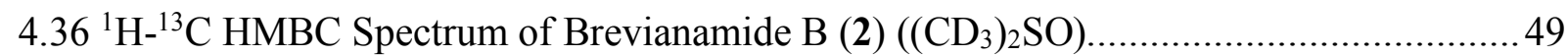

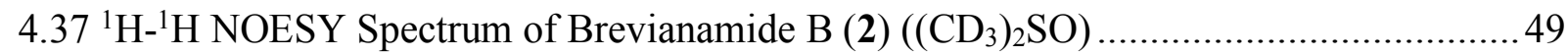

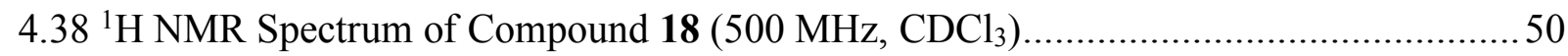

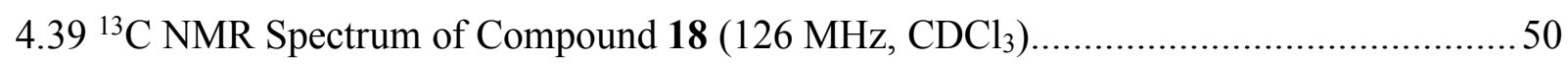

$4.40{ }^{1} \mathrm{H}$ NMR Spectrum of 3-Chloro-3-methyl-1-butyne (500 MHz, $\left.\mathrm{CDCl}_{3}\right)$.......................51

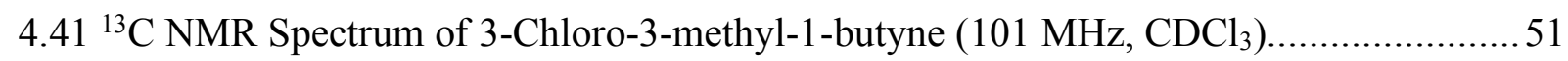

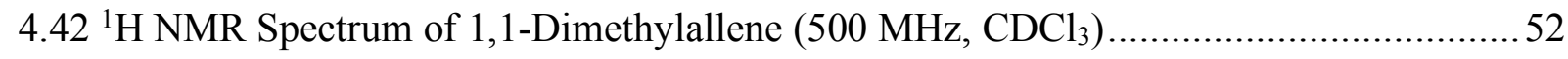

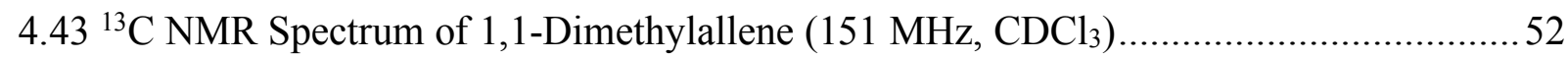

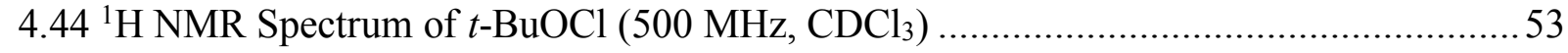

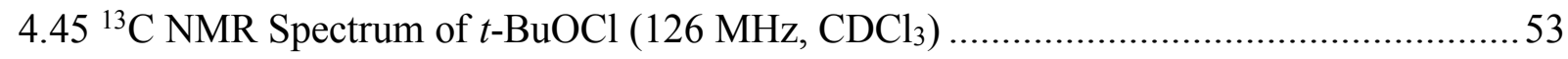

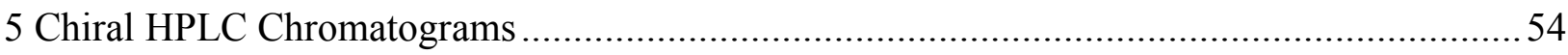

5.1 Chiral HPLC Chromatograms of Brevianamide A ................................................... 54

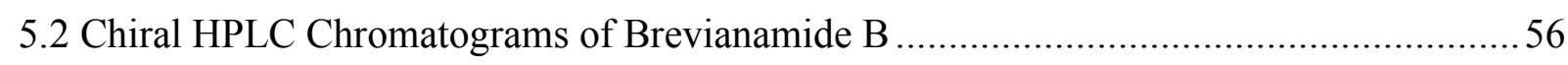

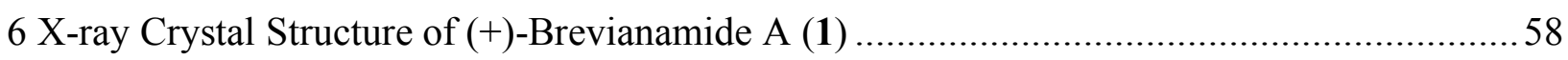


1. Overview of previous total and formal syntheses of brevianamide B.

NOTE: No synthesis of brevianamide A has been reported.

1.1 Williams' 1988 synthesis of (-)-brevianamide B (cationic cyclization)

- 18 steps

- $\quad 1.5-2.5 \%$ overall yield

- $0.8 \mathrm{mg}$ prepared

1.2 Williams' 1998 synthesis of $( \pm)$-brevianamide B (first biomimetic)

- 12 steps

- $2.8 \%$ overall yield

- $7.7 \mathrm{mg}$ prepared

1.3 Williams' 2006 synthesis of $( \pm)$-brevianamide B (late-stage indole formation)

- 12 steps

- $1.4 \%$ overall yield

- $4.0 \mathrm{mg}$ prepared

1.4 Williams' 2007 synthesis of ( \pm )-brevianamide B (second biomimetic)

- 14 steps

- $0.9 \%$ overall yield

- $4.7 \mathrm{mg}$ prepared

1.5 Simpkins' 2009 synthesis of (-)-brevianamide B (cationic double-cyclization)

- 9 steps

- $1.4 \%$ overall yield

- $6.0 \mathrm{mg}$ prepared

1.6 Scheerer's 2016 formal synthesis of $( \pm)$-brevianamide B (first intermolecular [4+2])

- 13 steps $(11+2$ formal steps $)$

- $\quad 2.9 \%$ overall yield $(6.4 \% \times 45 \%$ formal steps $)$

- $7.1 \mathrm{mg}$ of final intermediate

1.7 Scheerer's 2017 formal synthesis of $( \pm)$-brevianamide B (second intermolecular [4+2])

- 15 steps $(13+2$ formal steps $)$

- $2.1 \%$ overall yield $(4.6 \% \times 45 \%$ formal steps $)$

- $\quad 5.0 \mathrm{mg}$ of final intermediate 


\subsection{Williams' 1988 synthesis of (-)-brevianamide B (cationic cyclization approach)}

- 18 steps

- $1.5-2.5 \%$ overall yield

- $0.8 \mathrm{mg}$ prepared

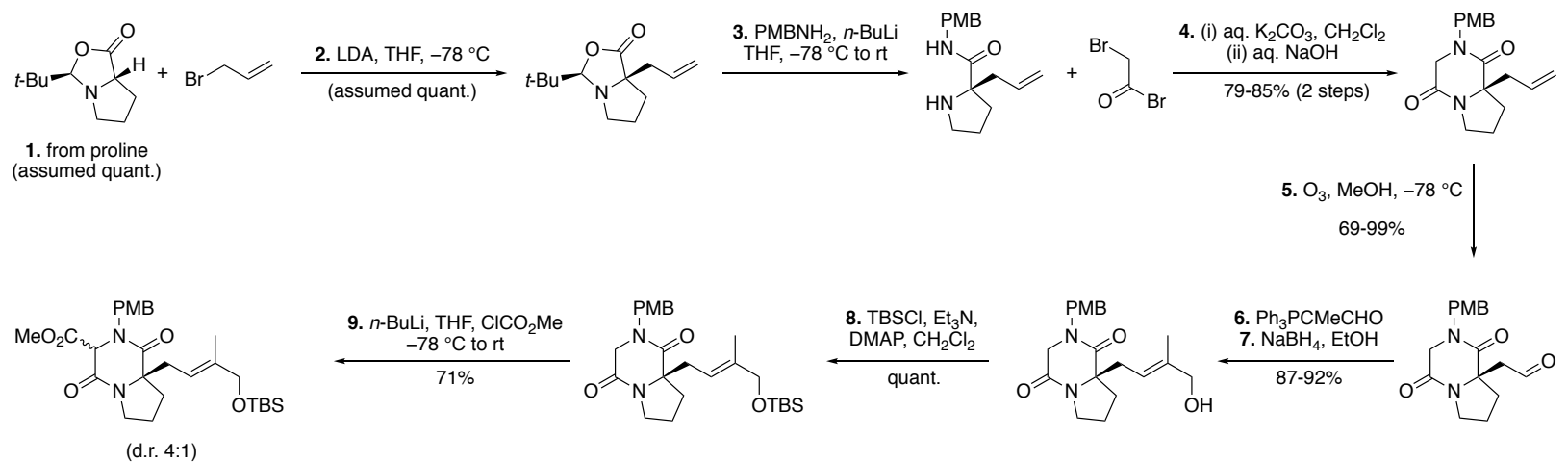

10. $\underset{82{ }_{3}^{\circ} \mathrm{P}, \mathrm{MeCN}}{62 \%}$
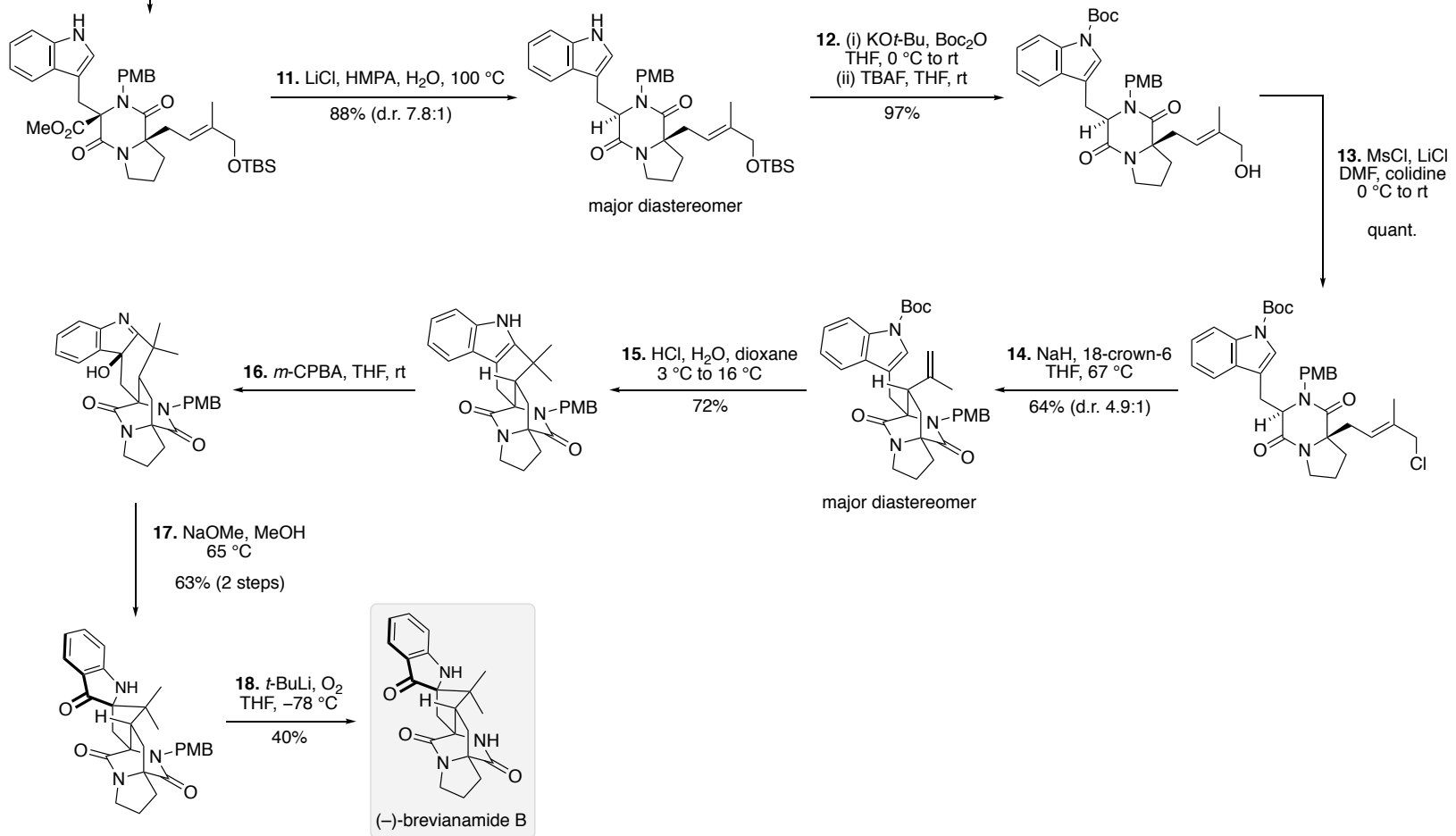

major diastereomer

\section{References:}

1. Williams, R. M., Glinka, T. \& Kwast, E. Facial selectivity of the intramolecular $\mathrm{S}_{\mathrm{N}} 2^{\prime}$ cyclization: stereocontrolled total synthesis of brevianamide B. J. Am. Chem. Soc. 110, 5927 5929 (1988).

2. Williams, R. M. \& Kwast, E. Carbanion-mediated oxidative deprotection of non-enolizable benzylated amides. Tetrahedron Lett. 30, 451-454 (1989)

3. Williams, R. M., Glinka, T., Kwast, E., Coffman, H. \& Stille, J. K. Asymmetric, stereocontrolled total synthesis of (-)-brevianamide B. J. Am. Chem. Soc. 112, 808-821 (1990). 


\subsection{Williams' 1998 synthesis of ( \pm )-brevianamide B (first biomimetic approach)}

- 12 steps

- $2.8 \%$ overall yield

- $7.7 \mathrm{mg}$ prepared

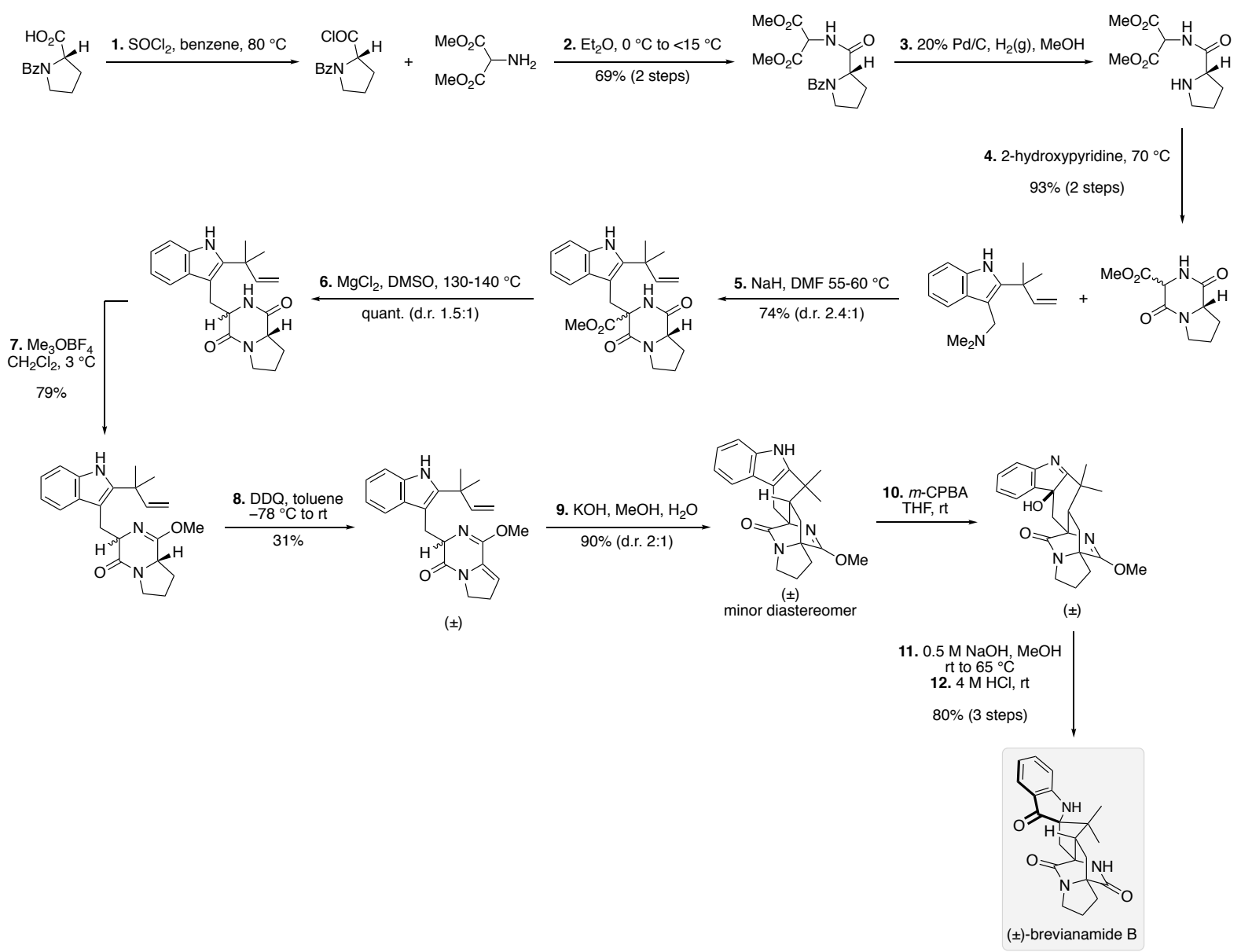

\section{References:}

1. Williams, R. M., Sanz-Cervera, J. F., Sancenón, F., Marco, J. A. \& Halligan, K. Biomimetic Diels-Alder cyclizations for the construction of the brevianamide, paraherquamide sclerotamide, and VM55599 ring systems. J. Am. Chem. Soc. 120, 1090-1091 (1998).

2. Williams, R. M., Sanz-Cervera, J. F., Sancenón, F., Marco, J. A. \& Halligan, K. Biomimetic Diels-Alder cyclizations for the construction of the brevianamide, paraherquamide, sclerotamide, asperparaline and VM55599 ring systems. Bioorg. Med. Chem. 6, 1233-1241 (1998).

3. Steps 1-6 taken from; Kametani, T., Kanaya, N. \& Ihara, M. Studies on the syntheses of heterocyclic compounds. Part 876. The chiral total synthesis of brevianamide $\mathrm{E}$ and deoxybrevianamide E. J. Chem. Soc. Perkin Trans. I 959-963 (1981). 
- 12 steps

- $1.4 \%$ overall yield

- $4.0 \mathrm{mg}$ prepared
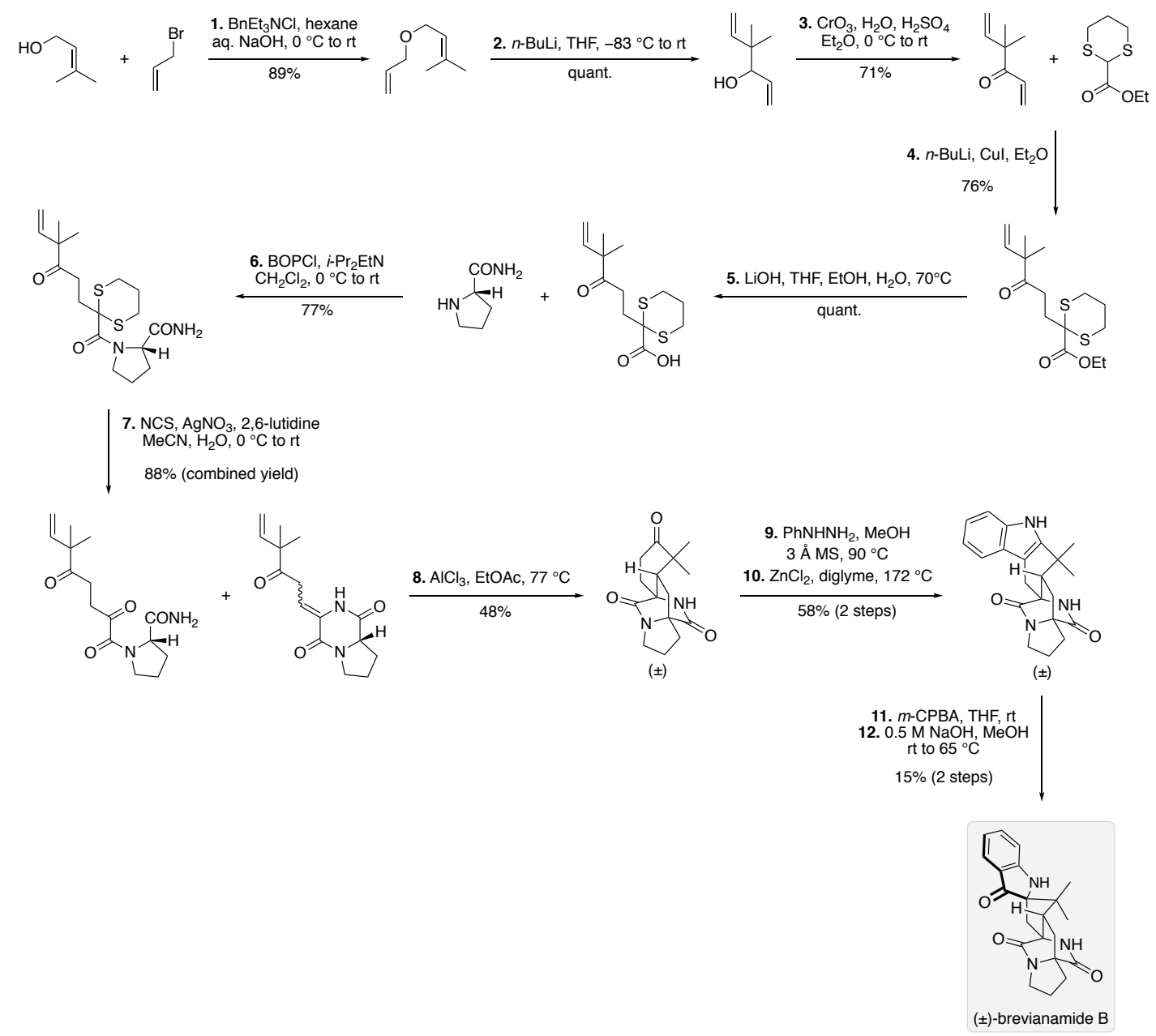

\section{References:}

1. Adams, L. A., Valente, M. W. N. \& Williams, R. M. A concise synthesis of d,1-brevianamide B via a biomimetically-inspired IMDA construction. Tetrahedron 62, 5195-5200 (2006).

2. Steps 1-3 taken from Dauben, W. G., Cogen, J. M., Ganzer, G. A. \& Behar, V. Photochemistry of 1,5-hexadien-3-ones: Wavelength-dependent selectivity in intramolecular enone-olefin photoadditions. J. Am. Chem. Soc. 113, 5817-5824 (1991). 


\subsection{Williams' 2007 synthesis of $( \pm)$-brevianamide B \& $( \pm)$-dehydrodeoxybreviaminde E}

(second biomimetic approach)

$( \pm)$-brevianamide B

- 14 steps

- $0.9 \%$ overall yield

- $4.7 \mathrm{mg}$ prepared

$( \pm)$-dehydrodeoxybreviaminde $\mathrm{E}$

- 12 steps

- $8 \%$ overall yield

- $98 \mathrm{mg}$ prepared
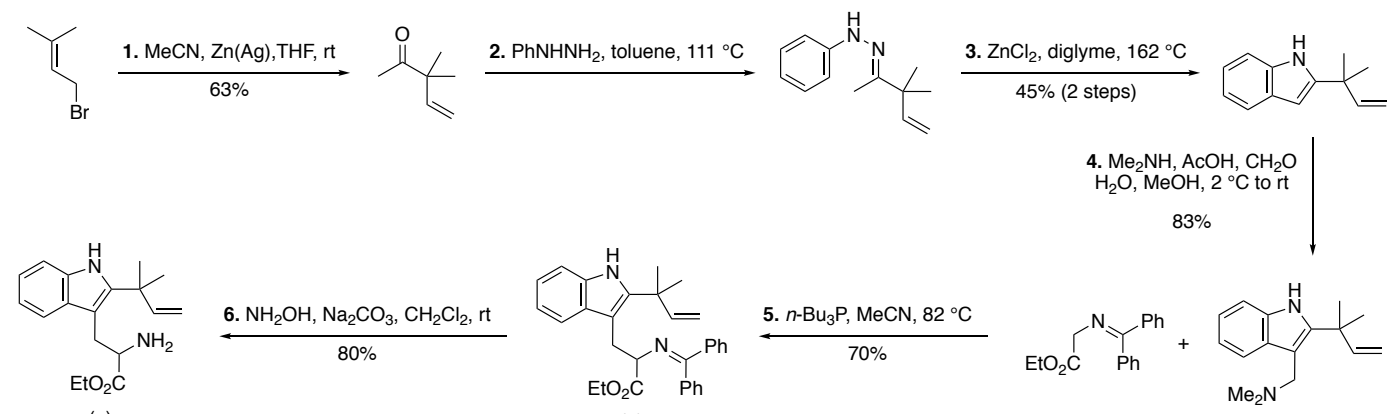

7. $\mathrm{Boc}_{2} \mathrm{O}, \mathrm{NaOH}$, dioxane, $\mathrm{rt}$ 8. $\mathrm{LiOH}, \mathrm{THF}, \mathrm{H}_{2} \mathrm{O}$, r

$78 \%$ (2 steps)

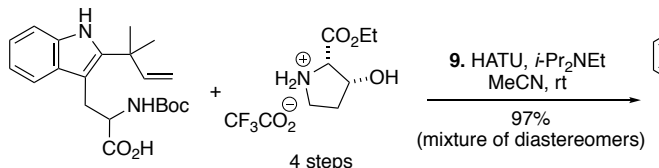

$( \pm)$

4 steps
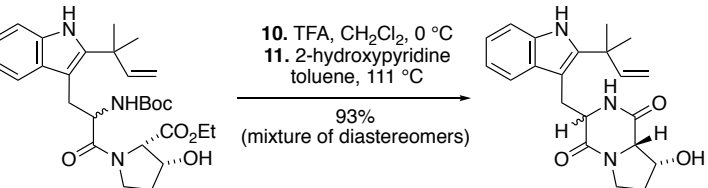

$( \pm)$
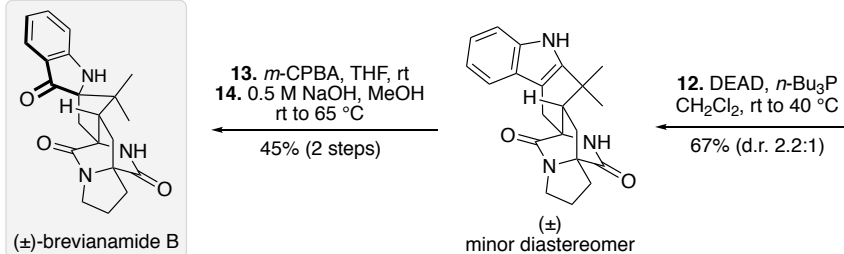

$67 \%$ (d.r. 2.2:1)

(土)

12. DEAD, $n-\mathrm{Bu}_{3} \mathrm{P}$ $\mathrm{CH}_{2} \mathrm{Cl}_{2}$, rt

$86 \%$

minor diastereomer

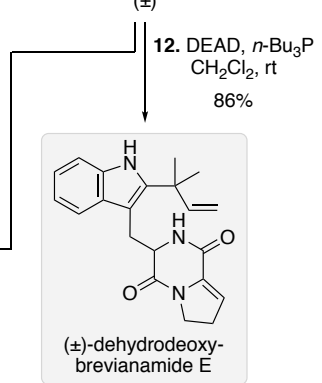

\section{References:}

1. Greshock, T. J. \& Williams, R. M. Improved biomimetic total synthesis of d,1-stephacidin A. Org. Lett. 9, 4255-4258 (2007).

2. Steps 1-4 taken from; Sanz-Cervera, J. F., Glinka, T. \& Williams, R. M. Biosynthesis of brevianamides A and B: In search of the biosynthetic Diels-Alder construction. Tetrahedron 49, 8471-8482 (1993). (which used ${ }^{3} \mathrm{H}_{2} \mathrm{CO}$ in step 4)

3. Steps 5-8 taken from; Stocking, E. M., Sanz-Cervera, J. F. \& Williams, R. M. Total Synthesis of VM55599. Utilization of an Intramolecular Diels-Alder Cycloaddition of Potential Biogenetic Relevance. J. Am. Chem. Soc. 122, 1675-1683 (2000). 
- 9 steps

- $1.4 \%$ overall yield

- $6.0 \mathrm{mg}$ prepared
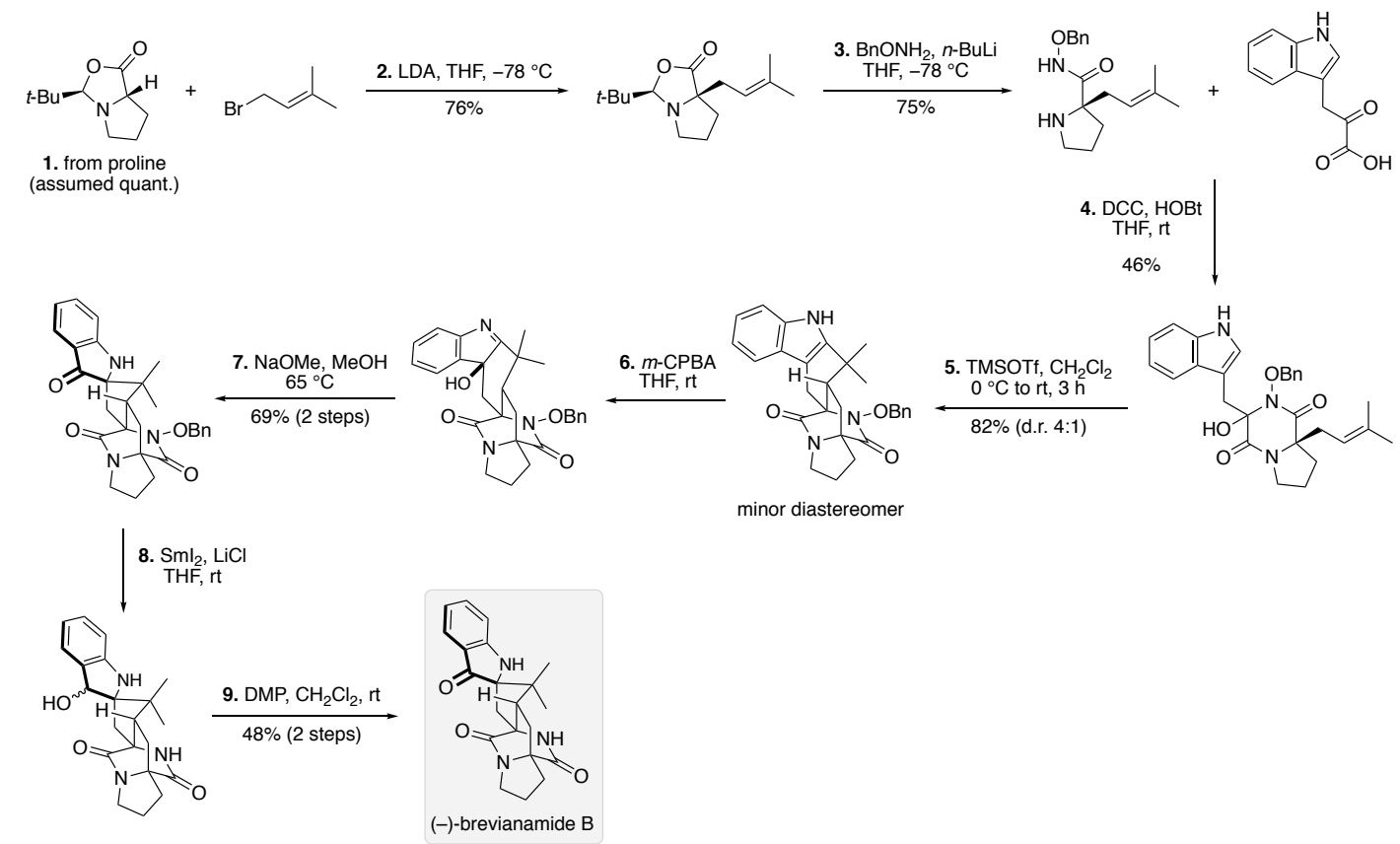

\section{References:}

1. Frebault, F. C., Simpkins, N. S. \& Fenwick, A. A concise enantioselective synthesis of entmalbrancheamide B. J. Am. Chem. Soc. 131, 4214-4215 (2009).

2. Frebault, F. C. \& Simpkins, N. S. A cationic cyclisation route to prenylated indole alkaloids: synthesis of malbrancheamide B and brevianamide B, and progress towards stephacidin A. Tetrahedron 66, 6585-6596 (2010). 


\subsection{Scheerer's 2016 formal synthesis of ( \pm )-brevianamide B (first intermolecular [4+2]}

approach)

- 13 steps $(11+2$ formal steps $)$

- $2.9 \%$ overall yield $(6.4 \% \times 45 \%$ formal steps $)$

- $7.1 \mathrm{mg}$ of final intermediate

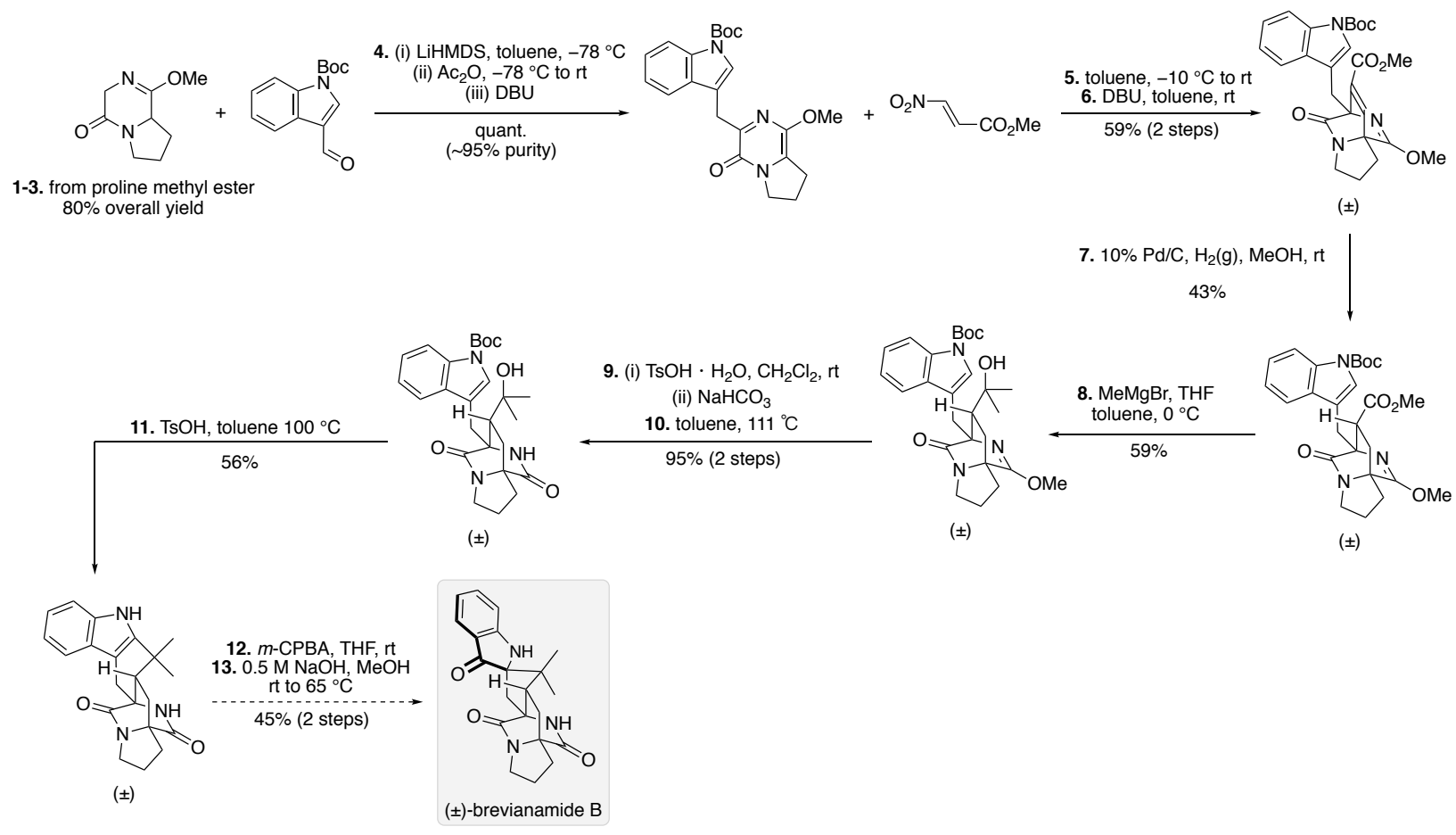

\section{References:}

1. Robins, J. G., Kim, K. J., Chinn, A. J., Woo, J. S. \& Scheerer, J. R. Intermolecular Diels-Alder Cycloaddition for the Construction of Bicyclo[2.2.2]diazaoctane Structures: Formal Synthesis of Brevianamide B and Premalbrancheamide. J. Org. Chem. 81, 2293-2301 (2016).

2. Steps 1-3 taken from; Laws, S. W. \& Scheerer, J. R. Enantioselective Synthesis of (+)Malbrancheamide B. J. Org. Chem. 78, 2422-2429 (2013).

3. Steps 12-13 taken from; Greshock, T. J. \& Williams, R. M. Improved biomimetic total synthesis of d,1-stephacidin A. Org. Lett. 9, 4255-4258 (2007). 


\subsection{Scheerer's 2017 formal synthesis of $( \pm)$-brevianamide B (second intermolecular [4+2] approach)}

- 15 steps $(13+2$ formal steps $)$

- $2.1 \%$ overall yield $(4.6 \% \times 45 \%$ formal steps $)$

- $5.0 \mathrm{mg}$ of final intermediate
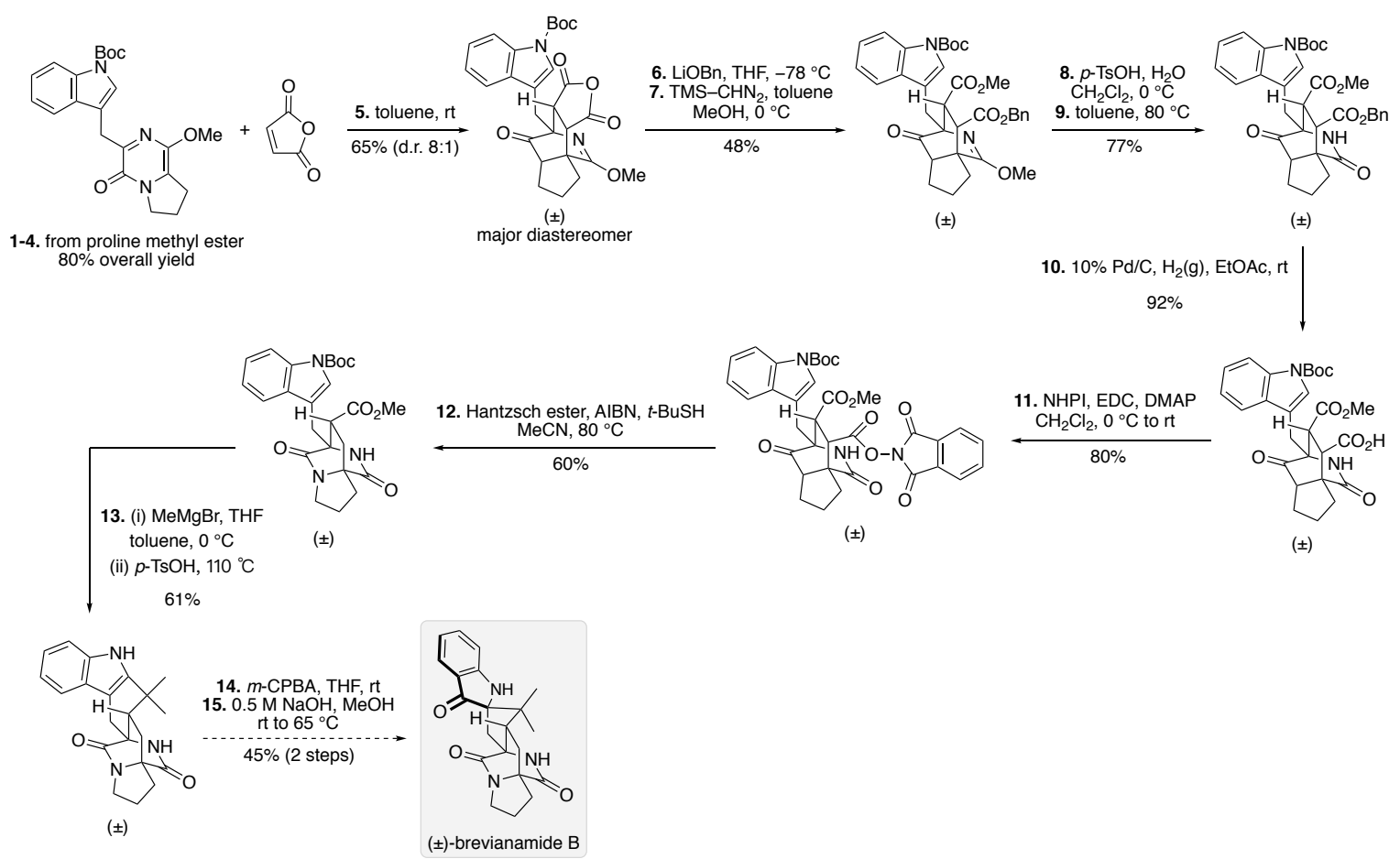

\section{References:}

1. Perkins, J. C., Wang, X., Pike, R. D. \& Scheerer, J. R. Further investigation of the intermolecular Diels-Alder cycloaddition for the synthesis of bicyclo[2.2.2]diazaoctane alkaloids. J. Org. Chem. 82, 13656-13662 (2017).

2. Steps 1-3 taken from; Laws, S. W. \& Scheerer, J. R. Enantioselective Synthesis of (+)Malbrancheamide B. J. Org. Chem. 78, 2422-2429 (2013).

3. Step 4 taken from; Robins, J. G., Kim, K. J., Chinn, A. J., Woo, J. S. \& Scheerer, J. R. Intermolecular Diels-Alder Cycloaddition for the Construction of Bicyclo[2.2.2]diazaoctane Structures: Formal Synthesis of Brevianamide B and Premalbrancheamide. J. Org. Chem. 81, 2293-2301 (2016).

4. Steps 14-15 taken from; Greshock, T. J. \& Williams, R. M. Improved biomimetic total synthesis of d,1-stephacidin A. Org. Lett. 9, 4255-4258 (2007). 


\section{Quantitative analysis of brevianamide A and B in Penicillium brevicompactum}

The ratio of brevianamide A and B in Penicillium brevicompactum cultures was determined to be consistently $\gtrsim 90: 10$ by Campbell and co-workers (see tables below). ${ }^{1}$

Table 1. Brevianamide A and B production from P. brevicompactum colony growth: over culture, inoculum $4.8 \times 10^{6}$ spores per plate.

\begin{tabular}{cccc}
\hline $\begin{array}{c}\text { Time after inoculation } \\
\text { (h) }\end{array}$ & $\begin{array}{c}\text { Brevianamide A } \\
\text { (mg per plate) }\end{array}$ & $\begin{array}{c}\text { Brevianamide B } \\
\text { (mg per plate) }\end{array}$ & $\mathbf{A}: \mathbf{B}$ \\
\hline 72 & 0.07 & 0.01 & $87.5: 12.5$ \\
84 & 0.19 & 0.02 & $90.5: 9.5$ \\
96 & 0.21 & 0.02 & $91.3: 8.7$ \\
108 & 0.45 & 0.03 & $93.9: 6.1$ \\
120 & 0.42 & 0.04 & $91.3: 8.7$ \\
144 & 0.50 & 0.04 & $92.6: 7.4$ \\
168 & 0.52 & 0.04 & $92.9: 7.1$ \\
192 & 0.53 & 0.05 & $91.4: 8.6$ \\
216 & 0.49 & 0.04 & $92.5: 7.5$
\end{tabular}

Table 2. Brevianamide A and B production from P. brevicompactum colony growth: over culture without medium replacement, inoculum $3.6 \times 10^{6}$ spores per plate.

\begin{tabular}{cccc}
\hline $\begin{array}{c}\text { Time after inoculation } \\
\text { (h) }\end{array}$ & $\begin{array}{c}\text { Brevianamide A } \\
\text { (mg per plate) }\end{array}$ & $\begin{array}{c}\text { Brevianamide B } \\
\text { (mg per plate) }\end{array}$ & $\mathbf{A}: \mathbf{B}$ \\
\hline 72 & 0.18 & 0.02 & $90: 10$ \\
84 & 0.28 & 0.03 & $90.3: 9.7$ \\
96 & 0.14 & 0.01 & $93.3: 6.7$ \\
108 & 0.19 & 0.02 & $90.5: 9.5$ \\
120 & 0.67 & 0.06 & $91.8: 8.2$ \\
144 & 0.66 & 0.06 & $91.7: 8.3$
\end{tabular}

Table 3. Brevianamide A and B production from P. brevicompactum colony growth: over culture with continual medium replacement, inoculum $3.6 \times 10^{6}$ spores per plate.

\begin{tabular}{cccc}
\hline $\begin{array}{c}\text { Time after inoculation } \\
\text { (h) }\end{array}$ & $\begin{array}{c}\text { Brevianamide A } \\
\text { (mg per plate) }\end{array}$ & $\begin{array}{c}\text { Brevianamide B } \\
\text { (mg per plate) }\end{array}$ & A : B \\
\hline 72 & 0.12 & 0.01 & $92.3: 7.7$ \\
84 & 0.54 & 0.04 & $93.1: 6.9$ \\
96 & 0.84 & 0.08 & $91.3: 8.7$ \\
108 & 1.08 & 0.09 & $92.3: 7.7$ \\
120 & 1.27 & 0.13 & $90.7: 1.3$ \\
144 & 1.61 & 0.14 & $92: 8$
\end{tabular}

\section{References:}

1. Bird, B. A. \& Campbell, I. M. Brevianamides A and B are formed only after conidiation has begun in solid cultures of Penicillium brevicompactum. Appl. Environ. Microbiol. 42, 521-525 (1981). 


\section{Experimental Conditions}

NMR Spectroscopy: ${ }^{1} \mathrm{H}$ NMR spectra were recorded at $600 \mathrm{MHz}$ on a Bruker 600 spectrometer with a AVANCE $3 \mathrm{HD}$ console, at $500 \mathrm{MHz}$ on Ascend 500 spectrometers with AVANCE 3 and AVANCE 3HD consoles, and at $400 \mathrm{MHz}$ on a Bruker 400 spectrometer with AVANCE 3 console. Residual solvent peaks were used as an internal reference for ${ }^{1} \mathrm{H} N M R$ spectra $\left(\mathrm{CDCl}_{3} \delta 7.26 \mathrm{ppm}\right.$, $\mathrm{CD}_{3} \mathrm{OD} \delta 3.31 \mathrm{ppm}, \mathrm{CD}_{2} \mathrm{Cl}_{2} \delta 5.32 \mathrm{ppm}$, and $\left.\left(\mathrm{CD}_{3}\right)_{2} \mathrm{SO} \delta 2.50 \mathrm{ppm}\right)$. Coupling constants $(J)$ are quoted to the nearest $0.1 \mathrm{~Hz} .{ }^{13} \mathrm{C}$ NMR spectra were recorded at $151 \mathrm{MHz}$ on a Bruker 600 spectrometer with an AVANCE 3HD console, $126 \mathrm{MHz}$ on Ascend 500 spectrometers with AVANCE 3 and AVANCE 3HD consoles, and at $101 \mathrm{MHz}$ on a Bruker 400 spectrometer with AVANCE 3 console. Solvent peaks were used as an internal reference for ${ }^{13} \mathrm{C}$ NMR spectra $\left(\mathrm{CDCl}_{3} \delta 77.16 \mathrm{ppm}, \mathrm{CD}_{3} \mathrm{OD} \delta 49.00 \mathrm{ppm}, \mathrm{CD}_{2} \mathrm{Cl}_{2} \delta 54.00 \mathrm{ppm}\right.$, and $\left.\left(\mathrm{CD}_{3}\right)_{2} \mathrm{SO} \delta 39.52 \mathrm{ppm}\right)$. Assignment of ${ }^{1} \mathrm{H}$ and ${ }^{13} \mathrm{C}$ NMR signals was assisted by ${ }^{1} \mathrm{H}-{ }^{1} \mathrm{H}$ COSY, ${ }^{1} \mathrm{H}-{ }^{13} \mathrm{C}$ HSQC,${ }^{1} \mathrm{H}-{ }^{13} \mathrm{C}$ $\mathrm{HMBC}$ and ${ }^{1} \mathrm{H}-{ }^{1} \mathrm{H}$ NOESY experiments.

IR Spectroscopy: IR spectra were recorded as neat samples on a Shimadzu IR affinity-1 FTIR spectrometer fitted with an ATR attachment.

Mass Spectroscopy: EI mass spectra were recorded on a MAT 900 XP double focussing high resolution sector, run at $70 \mathrm{eV}$. ESI spectra were recorded on a Bruker microTOF, calibrated with sodium formate clusters, with data analysis using Data Analysis 4.1 (Bruker Daltonics).

Analytical TLC: TLC analyses were performed on Merck silica plates coated with silica gel 60 F254 $(0.2 \mathrm{~mm})$ and were visualised with UV light and by staining with $p$-anisaldehyde or $\mathrm{KMnO}_{4}$ standard TLC stain solutions, followed by heating.

Flash Chromatography: Flash chromatography was performed using Merck silica gel 60 (40-63 $\mu \mathrm{m})$ for routine separations and Fluorochem silica gel $60(20-45 \mu \mathrm{m})$ for more challenging separations. Neutralised silica refers to columns packed with solvent containing $1 \% \mathrm{NEt}_{3}$ and rinsed with between 1 and 2 column volumes of solvent (free of $\mathrm{NEt}_{3}$ ) before use.

Optical Rotation: Optical rotations were recorded using a Bellingham Stanley ADP450 polarimeter with a Bellingham Stanley $0.5 \mathrm{~mL}$ cell $(l=0.25 \mathrm{dm})$. Concentrations $(c)$ are reported in $\mathrm{g} / 100 \mathrm{~mL}$.

Chiral HPLC: Analytical chiral HPLC was conducted using a modular Shimadzu system using a LC-20AD pump, DGU-20A 5 R degassing unit, SIL-20A HT autosampler, CTO-20A column oven, SPD-20A UV/Vis detector and CDM-20A communications module.

Experimental Procedures, Reagents and Solvents: Unless stated otherwise, reactions were performed under a positive pressure of dry nitrogen using anhydrous solvents. Commercially available chemicals were used as received, unless specified otherwise. Solvents and reagents dried over $4 \AA$ molecular sieves were dried for at least $24 \mathrm{~h}$ before use. Distillates were collected in flasks cooled with a dry ice/acetone bath to avoid loss of material. For reactions performed under anhydrous reaction conditions the reaction vessels were dried with a heat gun under vacuum prior to use. For anhydrous reactions, $\mathrm{NEt}_{3}$ was dried over activated $4 \mathrm{~A}$ molecular sieves for at least $24 \mathrm{~h}$ before use. 

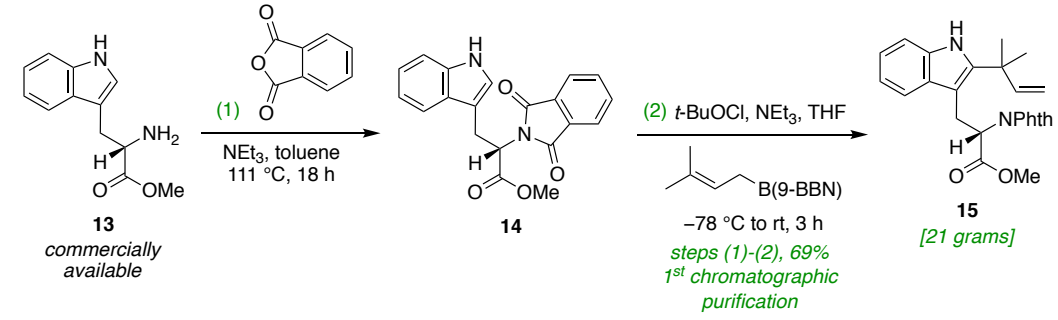

Step (1) - Based on conditions reported by Vederas and co-workers. ${ }^{1}$ To a stirred suspension of L-tryptophan methyl ester hydrochloride $(25.0 \mathrm{~g}, 98.2 \mathrm{mmol})$ and phthalic anhydride $(14.5 \mathrm{~g}$, $98.2 \mathrm{mmol})$ in toluene $(1000 \mathrm{~mL})$ was added $\mathrm{NEt}_{3}(27.4 \mathrm{~mL}, 196 \mathrm{mmol})$ and the mixture heated at reflux for $18 \mathrm{~h}$. The reaction was allowed to cool to room temperature and a mixture of saturated aq. $\mathrm{NH}_{4} \mathrm{Cl}(150 \mathrm{~mL})$ and water $(150 \mathrm{~mL})$ was added. The organic phase was separated, washed with brine $(300 \mathrm{~mL})$, dried $\left(\mathrm{Na}_{2} \mathrm{SO}_{4}\right)$ and concentrated under reduced pressure to yield crude $\mathbf{1 4}$ (33.6 g, 98\% crude mass recovery) which was used without further purification in the next step. A small sample was purified by flash chromatography (2:3 EtOAc/petroleum spirit) to give compound $\mathbf{1 4}$ as a pale-yellow foam suitable for characterisation. All data for compound 14 matched literature values. ${ }^{1}$

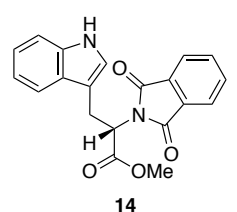

$\mathbf{R}_{\mathbf{f}} 0.24$ (2:3 EtOAc/petroleum spirit)

${ }^{1} \mathbf{H}$ NMR $\left(500 \mathrm{MHz}, \mathrm{CDCl}_{3}\right) \delta 7.97$ (br. s, $\left.1 \mathrm{H}\right), 7.74$ (dd, $\left.J=5.5,3.1 \mathrm{~Hz}, 2 \mathrm{H}\right), 7.65$ (dd, $J=5.5$, $3.0 \mathrm{~Hz}, 2 \mathrm{H}$ ), 7.60 (app. dt, $J=7.9,0.9 \mathrm{~Hz}, 1 \mathrm{H}$ ), 7.25 (app. dt, $J=7.3,0.9,0.9 \mathrm{~Hz}, 1 \mathrm{H}$ ), 7.12 (ddd, $J=8.1,7.0,1.2 \mathrm{~Hz}, 1 \mathrm{H}), 7.05(\mathrm{ddd}, J=8.0,7.0,1.1 \mathrm{~Hz}, 1 \mathrm{H}), 6.99(\mathrm{~d}, J=2.3 \mathrm{~Hz}, 1 \mathrm{H}), 5.28(\mathrm{dd}, J$ $=9.9,6.1 \mathrm{~Hz}, 1 \mathrm{H}), 3.79(\mathrm{~s}, 3 \mathrm{H}), 3.78-3.70(\mathrm{~m}, 2 \mathrm{H}) \mathrm{ppm}$;

${ }^{13} \mathbf{C}$ NMR $\left(126 \mathrm{MHz}, \mathrm{CDCl}_{3}\right) \delta 169.8,167.7,136.2,134.1,131.8,127.3,123.5,122.7,122.2$, 119.6, 118.6, 111.2, 111.2, 53.0, 52.7, 24.9 ppm;

IR (film, cm cm $^{-1}$ 3404, 3057, 3013, 2953, 2924, 2849, 1775, 1740, 1705, 1612, 1555;

HRMS $\left(\mathrm{ESI}^{+}\right.$) $\mathrm{m} / z 349.1211$ (calculated $[\mathrm{M}+\mathrm{H}]^{+}$349.1183), 371.1033 (calculated $[\mathrm{M}+\mathrm{Na}]^{+}$ 371.1002)

$[\boldsymbol{\alpha}]_{D}^{23.3}-201.1^{\circ}\left(c 1.07, \mathrm{CHCl}_{3}\right)$

\section{Reference:}

1. Liu, H., Pattabiraman, V. R. \& Vederas, J. C. Stereoselective Syntheses of 4-Oxa Diaminopimelic Acid and Its Protected Derivatives via Aziridine Ring Opening. Org. Lett. 9, 4211-4214 (2007). 

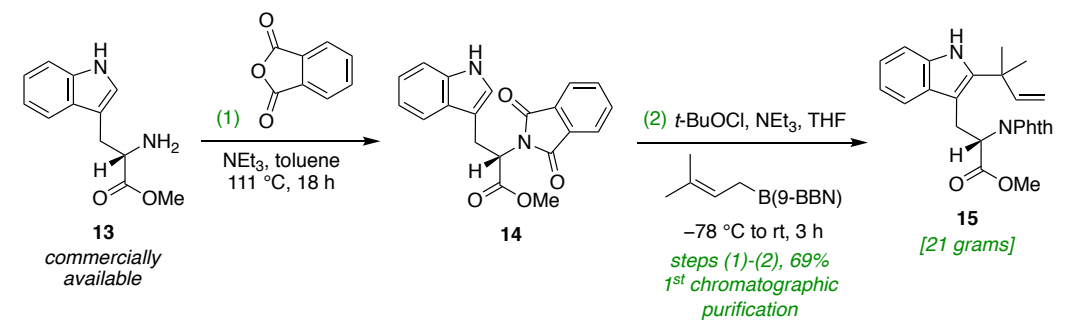

Step (2) - Based on a procedure reported by Danishefsky and co-workers. ${ }^{1}$ Reaction performed under anhydrous reaction conditions. A solution of crude compound 14 (25.0 g) and dry NEt 3 $(12.0 \mathrm{~mL}, 86.1 \mathrm{mmol})$ in THF $(250 \mathrm{~mL})$ was cooled to $-78^{\circ} \mathrm{C}$, and the lights in the vicinity of the reaction were turned off. $t$ - $\mathrm{BuOCl}(9.7 \mathrm{~mL}, 86.1 \mathrm{mmol}$, see page 30 for preparation) was added dropwise and the reaction stirred for 30 minutes at $-78^{\circ} \mathrm{C}$. A solution of $B$-Prenyl-9-BBN (360 $\mathrm{mL}, 180 \mathrm{mmol}, 0.5 \mathrm{M}$ in THF, see pages $28-29$ for preparation) was added dropwise at $-78{ }^{\circ} \mathrm{C}$, over $50 \mathrm{~min}$. After stirring for $1 \mathrm{~h}$ at $-78^{\circ} \mathrm{C}$ the cooling bath was removed and the reaction slowly allowed to warm to room temperature. Reaction progress was monitored by TLC analysis, following the disappearance of starting material 14, $\mathrm{R}_{\mathrm{f}} 0.24(1: 1 \mathrm{EtOAc} /$ petroleum spirit) and appearance of an intermediate with $\mathrm{R}_{\mathrm{f}} 0.63$ (1:1 EtOAc/petroleum spirit). After stirring for $2 \mathrm{~h}$ saturated aq. $\mathrm{K}_{2} \mathrm{CO}_{3}(75 \mathrm{~mL})$ was added. The layers were separated and the aqueous layer extracted with EtOAc $(3 \times 125 \mathrm{~mL})$. The combined organic layers were dried $\left(\mathrm{MgSO}_{4}\right)$ and concentrated under reduced pressure. The crude product was filtered through silica with $\mathrm{CH}_{2} \mathrm{Cl}_{2}$, concentrated and purified by flash chromatography (1:9 to 1:3 EtOAc/petroleum spirit) to give compound $\mathbf{1 5}$ (21.1 g, $50.7 \mathrm{mmol}, 69 \%$ over 2 steps) as a pale-yellow foam. All spectroscopic data matched

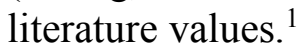

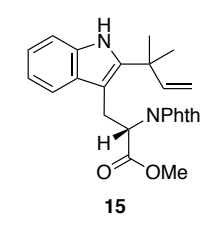

$\mathbf{R}_{\mathbf{f}} 0.55$ (1:1 EtOAc/petroleum spirit), 0.24 (1:4 EtOAc/petroleum spirit);

${ }^{1} \mathbf{H}$ NMR $\left(600 \mathrm{MHz}, \mathrm{CDCl}_{3}\right) \delta 7.86$ (br. s, $\left.1 \mathrm{H}\right), 7.69(\mathrm{dd}, J=5.5,3.0 \mathrm{~Hz}, 2 \mathrm{H}), 7.62(\mathrm{dd}, J=5.5$, $3.0 \mathrm{~Hz}, 2 \mathrm{H}$ ), 7.28 (app. dt, $J=7.9,0.9 \mathrm{~Hz}, 1 \mathrm{H}$ ), 7.13 (app. dt, $J=8.0,0.8 \mathrm{~Hz}, 1 \mathrm{H}$ ), 6.90 (ddd, $J=$ $8.1,7.0,1.1 \mathrm{~Hz}, 1 \mathrm{H}), 6.71$ (ddd, $J=8.0,7.0,1.0 \mathrm{~Hz}, 1 \mathrm{H}), 6.19(\mathrm{dd}, J=17.5,10.5 \mathrm{~Hz}, 1 \mathrm{H}), 5.23-$ $5.17(\mathrm{~m}, 2 \mathrm{H}), 5.15(\mathrm{dd}, J=10.5,1.0 \mathrm{~Hz}, 1 \mathrm{H}), 3.86(\mathrm{dd}, J=15.4,3.9 \mathrm{~Hz}, 1 \mathrm{H}), 3.78(\mathrm{~s}, 3 \mathrm{H}), 3.67$ (dd, $J=15.4,11.3 \mathrm{~Hz}, 1 \mathrm{H}), 1.58(\mathrm{~s}, 3 \mathrm{H}), 1.57$ (s, 3H) ppm;

${ }^{13}$ C NMR $\left(151 \mathrm{MHz}, \mathrm{CDCl}_{3}\right) \delta 169.7,167.8,146.0,140.3,134.0,134.0,132.0,129.9,123.3$, $121.3,119.3,117.9,112.3,110.3,106.4,53.6,52.9,39.3,27.8,27.7,24.6$ ppm;

$[\boldsymbol{\alpha}]_{D}^{23.7}-221.6^{\circ}\left(c 3.97, \mathrm{CHCl}_{3}\right)$, literature: $[\boldsymbol{\alpha}]_{D}^{25}-180.8^{\circ}\left(c 3.9, \mathrm{CHCl}_{3}\right) .^{1}$

\section{Reference:}

1. Schkeryantz, J. M., Woo, J. C. G., Siliphaivanh, P., Depew, K. M. \& Danishefsky, S. J. Total synthesis of gypsetin, deoxybrevianamide E, brevianamide E, and tryprostatin B: Novel constructions of 2,3-disubstituted indoles. J. Am. Chem. Soc. 121, 11964-11975 (1999). 
3.3 Experimental Procedure for Compound 16

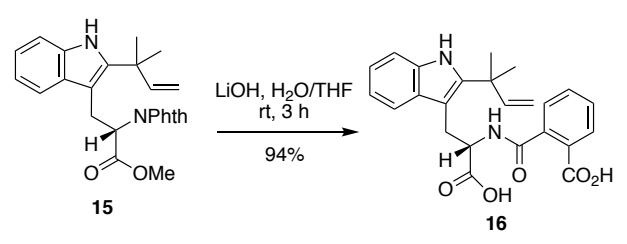

Compound 16 was prepared based on conditions reported by Ley and co-workers. ${ }^{1}$ To a solution of compound $15(67 \mathrm{mg}, 0.16 \mathrm{mmol})$ in $1: 1 \mathrm{THF} / \mathrm{H}_{2} \mathrm{O}(2 \mathrm{~mL})$ was added solid $\mathrm{LiOH}(19 \mathrm{mg}$, $0.79 \mathrm{mmol})$. The solution was stirred at $\mathrm{rt}$ for $3 \mathrm{~h}$ before being neutralised with $1 \mathrm{M}$ aq. $\mathrm{HCl}$ and the aqueous layer extracted with $\mathrm{Et}_{2} \mathrm{O}(3 \times 5 \mathrm{~mL})$. The combined organics were washed with $\mathrm{H}_{2} \mathrm{O}$ $(2 \times 2.5 \mathrm{~mL})$, brine $(2.5 \mathrm{~mL})$, dried $\left(\mathrm{Na}_{2} \mathrm{SO}_{4}\right)$ and concentrated under reduced pressure to give crude compound 16 (62 $\mathrm{mg}, 0.15 \mathrm{mmol}, 94 \%$ crude mass recovery) as a yellow foam, which was sufficiently pure for characterisation purposes.

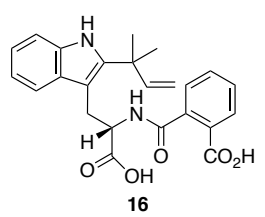

${ }^{1} \mathbf{H}$ NMR $\left(500 \mathrm{MHz}, \mathrm{CD}_{3} \mathrm{OD}\right) \delta 7.91(\mathrm{dd}, J=7.6,1.6 \mathrm{~Hz}, 1 \mathrm{H}), 7.60(\mathrm{~d}, J=7.9 \mathrm{~Hz}, 1 \mathrm{H}), 7.46$ (app. td, $J=7.6,1.6 \mathrm{~Hz}, 1 \mathrm{H}), 7.42$ (app. td, $J=7.5,1.6 \mathrm{~Hz}, 1 \mathrm{H}), 7.31(\mathrm{~d}, J=8.0 \mathrm{~Hz}, 1 \mathrm{H}), 7.04$ (ddd, $J$ $=8.1,6.9,1.2 \mathrm{~Hz}, 1 \mathrm{H}), 6.98-6.93(\mathrm{~m}, 1 \mathrm{H}), 6.89(\mathrm{dd}, J=7.4,1.5 \mathrm{~Hz}, 1 \mathrm{H}), 6.26(\mathrm{dd}, J=17.5,10.6$ $\mathrm{Hz}, 1 \mathrm{H}), 5.17$ (dd, $J=17.4,1.3 \mathrm{~Hz}, 1 \mathrm{H}), 5.11$ (dd, $J=10.5,1.2 \mathrm{~Hz}, 1 \mathrm{H}), 4.98$ (app. t, $J=7.8 \mathrm{~Hz}$, $1 \mathrm{H}), 3.51(\mathrm{dd}, J=14.5,7.7 \mathrm{~Hz}, 1 \mathrm{H}), 3.27$ (dd, $J=14.5,7.9 \mathrm{~Hz}, 1 \mathrm{H}), 1.59$ (s, 3H), 1.59 (s, 3H) ppm;

${ }^{13}$ C NMR (126 MHz, CD $\left.{ }_{3} \mathrm{OD}\right) \delta 175.3,172.2,169.2,147.6,142.1,139.4,136.4,132.9,131.2$, $131.1,130.5,130.4,129.0,121.7,119.6,119.3,112.1,111.6,106.6,55.6,40.4,28.9,28.5,28.4$ ppm;

IR (film, $\mathrm{cm}^{-1}$ ) 3000, 1771, 1694, 1597, 1525, 1462, 1389;

HRMS $\left(\mathrm{EI}^{+}\right) \mathrm{m} / \mathrm{z} 420.16655$ (calculated $[\mathrm{M}]^{+}:$420.16797);

$[\boldsymbol{\alpha}]_{D}^{23.4}-28.3^{\circ}(c 0.10, \mathrm{MeOH})$.

\section{Reference:}

1. Hewitt, P. R., Cleator, E. \& Ley, S. V. A concise total synthesis of (+)-okaramine C. Org. Biomol. Chem. 2, 2415-2417 (2004). 

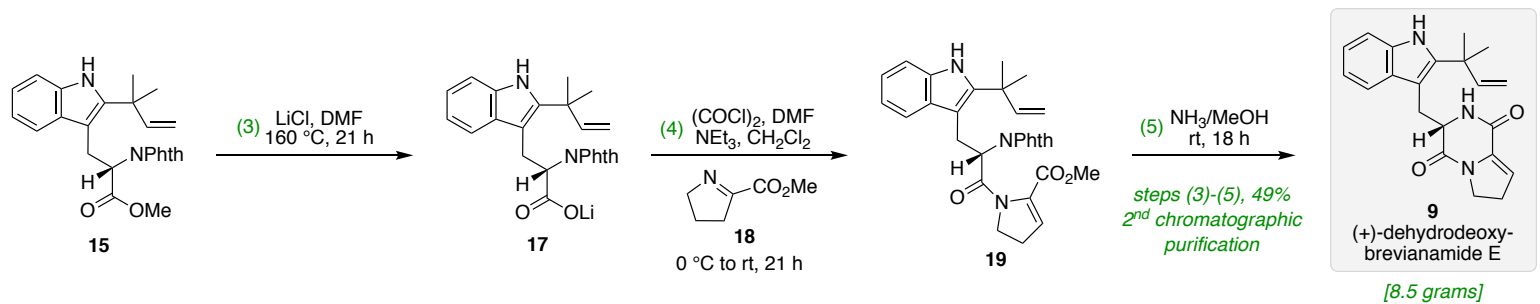

Step (3) - Inspired by the work of Hell, ${ }^{1,2}$ and Fisher. ${ }^{3}$ Compound 15 (24.6 g, 59.0 mmol) and $\mathrm{LiCl}(50.0 \mathrm{~g}, 1180 \mathrm{mmol})$ were suspended in DMF $(125 \mathrm{~mL})$ and the resulting mixture heated at $160{ }^{\circ} \mathrm{C}$ for $21 \mathrm{~h}$ under a flow of nitrogen. The thick brown suspension was diluted with $\mathrm{CH}_{2} \mathrm{Cl}_{2}$ $(300 \mathrm{~mL})$, sonicated for 30 minutes and the solid removed by filtration. The residual solid was broken up, suspended in $\mathrm{CH}_{2} \mathrm{Cl}_{2}(300 \mathrm{~mL})$, stirred for 30 minutes and filtered. The combined filtrates were concentrated under reduced pressure, then dried at $160{ }^{\circ} \mathrm{C}$ under reduced pressure. The crude product was re-dissolved in $\mathrm{CH}_{2} \mathrm{Cl}_{2}$, filtered and concentrated twice to remove any residual $\mathrm{LiCl}$ solid, before being dried at $160{ }^{\circ} \mathrm{C}$ under reduced pressure to give crude lithium carboxylate 17 (23.6 g), which was used without further purification in the next step.

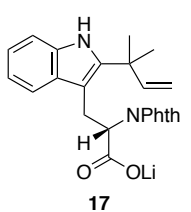

${ }^{1}$ H NMR (500 MHz, CD $\left.{ }_{3} \mathrm{OD}\right) \delta 7.62$ (app. s, 4H), 7.21 (app. dt, $\left.J=8.1,0.9 \mathrm{~Hz}, 1 \mathrm{H}\right), 7.11$ (app. t, $J=8.0,0.9 \mathrm{~Hz}, 1 \mathrm{H}), 6.75(\mathrm{ddd}, J=8.1,7.0,1.1 \mathrm{~Hz}, 1 \mathrm{H}), 6.53(\mathrm{ddd}, J=8.0,7.0,1.0 \mathrm{~Hz} 1 \mathrm{H}), 6.26$ $(\mathrm{dd}, J=17.4,10.6 \mathrm{~Hz}, 1 \mathrm{H}), 5.13(\mathrm{dd}, J=17.4,1.2 \mathrm{~Hz}, 1 \mathrm{H}), 5.04(\mathrm{dd}, J=10.6,1.2 \mathrm{~Hz}, 1 \mathrm{H}), 4.95$ (dd, $J=12.0,3.3 \mathrm{~Hz}, 1 \mathrm{H}), 3.84(\mathrm{dd}, J=15.3,3.3 \mathrm{~Hz}, 1 \mathrm{H}), 3.58(\mathrm{dd}, J=15.2,12.0 \mathrm{~Hz}, 1 \mathrm{H}), 1.59$ $(\mathrm{s}, 3 \mathrm{H}), 1.54(\mathrm{~s}, 3 \mathrm{H}) \mathrm{ppm}$;

${ }^{13}$ C NMR (126 MHz, CD $\left.{ }_{3} \mathrm{OD}\right) \delta 176.1,170.1,147.8,141.3,136.2,134.6,133.7,131.1,123.5$, $121.2,118.9,118.6,111.7,111.2,108.5,57.8,40.4,28.6,28.3,26.0$ ppm;

IR (film, $\mathrm{cm}^{-1}$ ) 2924, 2365, 1773, 1701, 1611, 1466, 1396, 1350;

HRMS $\left(\mathrm{ESI}^{-}\right) \mathrm{m} / z 401.1476$ (calculated $[\mathrm{M}-\mathrm{Li}]^{-}$401.1507);

$[\boldsymbol{\alpha}]_{D}^{22.9}-226.0^{\circ}(c 0.20, \mathrm{MeOH})$.

\section{References:}

1. Fölling, J., Belov, V., Kunetsky, R., Medda, R., Schönle, A., Egner, A., Eggeling, C., Bossi, M. \& Hell, S. W. Photochromic rhodamines provide nanoscopy with optical sectioning. Angew. Chem. Int. Ed. 46, 6266-6270 (2007).

2. Belov, V. N., Bossi, M. L., Fölling, J., Boyarskiy, V. P. \& Hell, S. W. Rhodamine spiroamides for multicolor single-molecule switching fluorescent nanoscopy. Chem. Eur. J. 15, 10762-10776 (2009).

3. Fisher, J. W. \& Trinkle, K. L. Iodide dealkylation of benzyl, PMB, PNB, and t-Butyl N-acyl amino acid esters via lithium ion coordination. Tetrahedron Lett. 35, 2505-2508 (1994). 

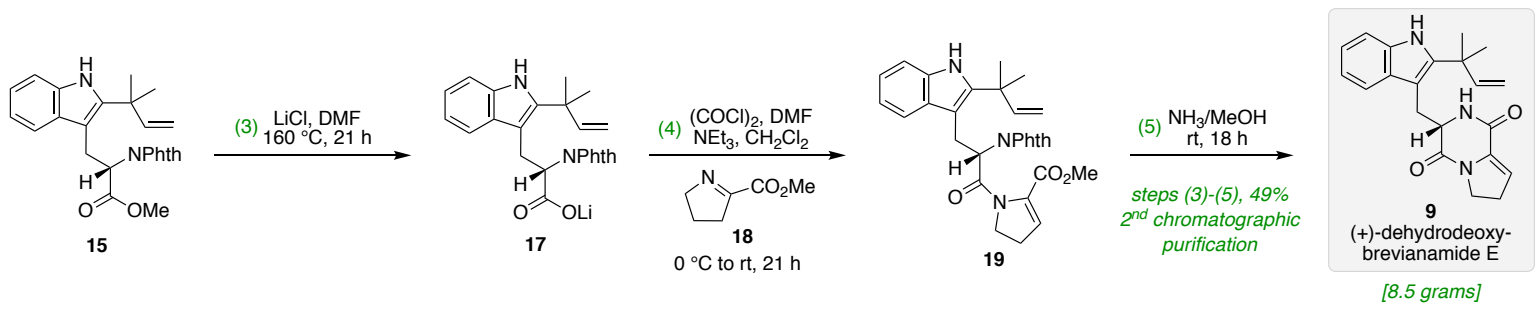

Step (4) - Inspired by the work of Schmalz, ${ }^{1}$ and Soai. ${ }^{2}$ Reaction performed under anhydrous reaction conditions. DMF $(1.90 \mathrm{~mL}, 24.6 \mathrm{mmol})$ was added dropwise to a solution of oxalyl chloride $(6.24 \mathrm{~mL}, 73.8 \mathrm{mmol})$ in $\mathrm{CH}_{2} \mathrm{Cl}_{2}(200 \mathrm{~mL})$ at $0{ }^{\circ} \mathrm{C}$. A suspension of crude compound 17 from Step (3) $(20.1 \mathrm{~g})$ in $\mathrm{CH}_{2} \mathrm{Cl}_{2}(200 \mathrm{~mL})$ was then added dropwise over $30 \mathrm{~min}$ at $0{ }^{\circ} \mathrm{C}$ and the residual 17 was washed into the reaction with $\mathrm{CH}_{2} \mathrm{Cl}_{2}(2 \times 50 \mathrm{~mL})$ and the resulting mixture was stirred at $0{ }^{\circ} \mathrm{C}$ for $30 \mathrm{~min}$. A solution of compound $18(5.84 \mathrm{~mL}, 49.2 \mathrm{mmol}$, see page 27 for preparation) and $\mathrm{NEt}_{3}(10.3 \mathrm{~mL}, 73.8 \mathrm{mmol})$ in $\mathrm{CH}_{2} \mathrm{Cl}_{2}$ was added dropwise over $20 \mathrm{~min}$ at $0{ }^{\circ} \mathrm{C}$. The cooling bath was then removed and the reaction stirred for $21 \mathrm{~h}$ at $\mathrm{rt}$. The reaction was quenched by the addition of $1 \mathrm{M}$ aq. $\mathrm{HCl}(80 \mathrm{~mL})$, the phases separated and the aqueous phase extracted with $\mathrm{CH}_{2} \mathrm{Cl}_{2}(3 \times 50 \mathrm{~mL})$. The combined organics were washed with saturated aq. $\mathrm{NaHCO}_{3}(80 \mathrm{~mL})$, and the aqueous layer then back extracted with $\mathrm{CH}_{2} \mathrm{Cl}_{2}(50 \mathrm{~mL})$. The combined organics were washed with brine $(120 \mathrm{~mL})$, dried $\left(\mathrm{Na}_{2} \mathrm{SO}_{4}\right)$ and concentrated under reduced pressure to give crude compound 19 (23.9 g) which was used without further purification in the next step. A small sample could be purified by flash chromatography (1:1 EtOAc/petroleum spirit) to give compound $\mathbf{1 9}$ as a lemon-yellow foam suitable for characterisation.

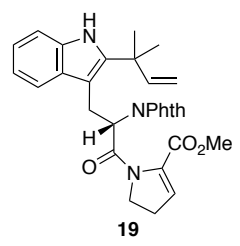

$\mathbf{R}_{\mathbf{f}} 0.28$ (1:1 EtOAc/petroleum spirit);

${ }^{1} \mathbf{H}$ NMR $\left(500 \mathrm{MHz}, \mathrm{CD}_{2} \mathrm{Cl}_{2}\right) \delta 7.95(\mathrm{~s}, 1 \mathrm{H}), 7.64$ (app. s, 4H), $7.17-7.13(\mathrm{~m}, 2 \mathrm{H}), 6.86$ (dd, $J=$ $8.2,7.1 \mathrm{~Hz}, 1 \mathrm{H}), 6.61$ (app. t, $J=7.4 \mathrm{~Hz}, 1 \mathrm{H}), 6.20$ (dd, $J=17.4,10.5 \mathrm{~Hz}, 1 \mathrm{H}), 5.87$ (dd, $J=3.5$, $2.5 \mathrm{~Hz}, 1 \mathrm{H}), 5.24(\mathrm{dd}, J=10.4,4.4 \mathrm{~Hz}, 1 \mathrm{H}), 5.20(\mathrm{dd}, J=17.5,1.1 \mathrm{~Hz}, 1 \mathrm{H}), 5.13(\mathrm{dd}, J=10.6$, $1.1 \mathrm{~Hz}, 1 \mathrm{H}), 3.94$ (br. s, 1H), 3.83 (dd, $J=15.4,4.4 \mathrm{~Hz}, 1 \mathrm{H}), 3.72$ (s, 3H), 3.62 (app. q, $J=10.5$ $\mathrm{Hz}, 1 \mathrm{H}), 3.46$ (dd, $J=15.4,10.4 \mathrm{~Hz}, 1 \mathrm{H}), 2.62$ (app. dtd, $J=17.6,10.0,2.5 \mathrm{~Hz}, 1 \mathrm{H}$ ), 2.39 (app. ddt, $J=17.5,10.6,3.4 \mathrm{~Hz}, 1 \mathrm{H}), 1.55(\mathrm{~s}, 3 \mathrm{H}), 1.55(\mathrm{~s}, 3 \mathrm{H})$;

${ }^{13}$ C NMR $\left(151 \mathrm{MHz}, \mathrm{CD}_{2} \mathrm{Cl}_{2}\right) \delta 167.8,167.7,162.1,146.6,140.9,137.0,134.5,134.5,131.9$, 130.3, 123.6, 123.5, 121.5, 119.4, 118.1, 112.3, 110.6, 106.6, 54.2 (beneath residual solvent peak), 52.6, 50.4 (br.), 39.7, 29.6, 27.9, 27.9, 25.4;

IR (film, $\mathrm{cm}^{-1}$ ) 1775, 1713, 1655, 1639, 1611, 1437, 1381;

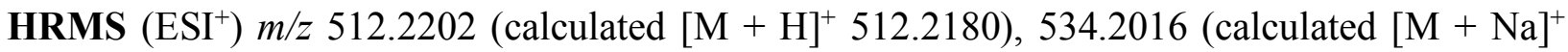
534.1999);

$[\boldsymbol{\alpha}]_{D}^{\mathbf{2 3 . 0}}-130.1^{\circ}(c 0.38, \mathrm{MeOH})$.

\section{References:}

1. Huy, P., Neudörfl, J.-M. \& Schmalz, H.-G. A practical synthesis of trans-3-substituted proline derivatives through 1,4-addition. Org. Lett. 13, 216-219 (2011).

2. Ookawa, A. \& Soai, K. Asymmetric synthesis of optically active threo- and erythropyrrolidinylbenzyl alcohol by the highly stereospecific arylation of $(S)$-proline and the subsequent highly diastereoselective reduction of the $\alpha$-amino ketone. J. Chem. Soc., Perkin Trans. $11465-$ 1465 (1987). 
3.6 Experimental Procedure for Compound 9
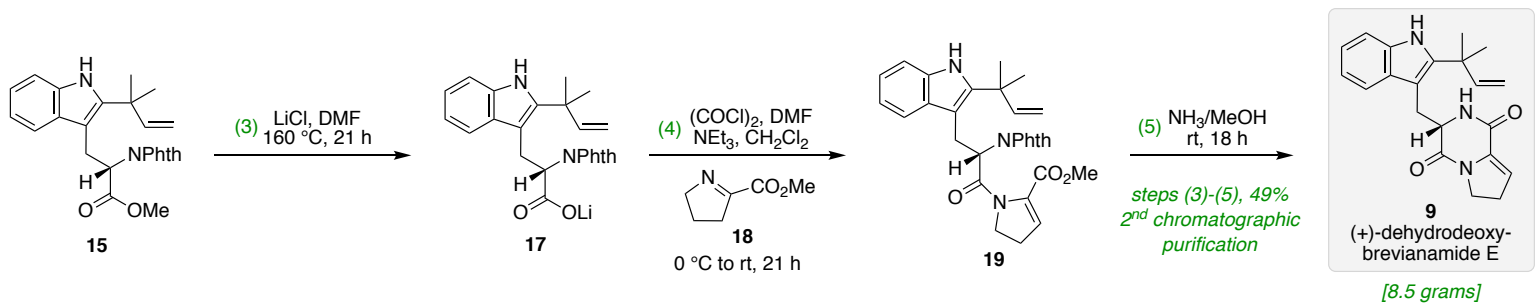

Step (5) - The crude product 19 (23.9 g) from Step (4) was dissolved in a solution of $\mathrm{NH}_{3}$ in $\mathrm{MeOH}$ $(7 \mathrm{M}, 1200 \mathrm{~mL})$ and stirred at room temperature for $18 \mathrm{~h}$. The crude reaction mixture was concentrated under reduced pressure. Flash chromatography on neutralised silica (7:3 to 8:2 EtOAc/petroleum spirit) gave (+)-dehydrodeoxybrevianamide E (9) (8.51 g, 24.4 mmol, 49\% over 3 steps) as a pale-yellow foam. All spectroscopic data matched literature values. ${ }^{1}$

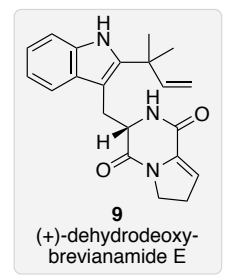

$\mathbf{R}_{\mathbf{f}} 0.19$ (8:2 EtOAc/petroleum spirit);

${ }^{1}$ H NMR (500 MHz, $\mathrm{CDCl}_{3}$ ) $\delta 8.08$ (br. s, 1H), 7.53 (d, $\left.J=7.9 \mathrm{~Hz}, 1 \mathrm{H}\right), 7.32$ (app. dt, $J=8.1,0.9$ $\mathrm{Hz}, 1 \mathrm{H}), 7.17$ (ddd, $J=8.1,7.1,1.2 \mathrm{~Hz}, 1 \mathrm{H}), 7.11(\mathrm{ddd}, J=8.1,7.1,1.1 \mathrm{~Hz}, 1 \mathrm{H}), 6.14$ (app. $\mathrm{t}, J=$ $3.1 \mathrm{~Hz}, 1 \mathrm{H}), 6.12$ (dd, $J=17.2,10.4 \mathrm{~Hz}, 1 \mathrm{H}), 5.66$ (br. s, 1H), $5.20-5.15$ (m, 2H), 4.52 (ddd, $J=$ $11.3,3.5,1.8 \mathrm{~Hz}, 1 \mathrm{H}), 4.14-4.02(\mathrm{~m}, 2 \mathrm{H}), 3.73(\mathrm{dd}, J=14.7,3.7 \mathrm{~Hz}, 1 \mathrm{H}), 3.23$ (dd, $J=14.6$, $11.3 \mathrm{~Hz}, 1 \mathrm{H}), 2.78$ (app. td, $J=9.1,3.1 \mathrm{~Hz}, 2 \mathrm{H}), 1.55$ (s, 3H), 1.55 (s, 3H) ppm;

${ }^{13} \mathrm{C}$ NMR $\left(126 \mathrm{MHz} \mathrm{CDCl}_{3}\right) \delta 162.7,156.6,145.8,141.8,134.4,133.2,128.9,122.3,120.2$, $118.9,118.3,112.6,110.9,104.6,57.6,45.7,39.2,30.9,28.1,28.0,27.9$ ppm;

IR (film, cm $\left.{ }^{-1}\right) 3331$ br., 2967, 2926, 1672, 1639, 1435;

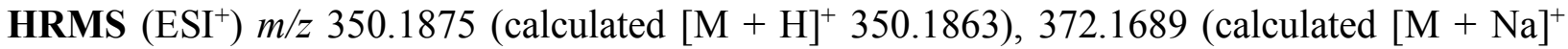
372.1682);

$[\boldsymbol{\alpha}]_{D}^{23.1}-33.2^{\circ}\left(c 1.30, \mathrm{CHCl}_{3}\right)$, literature: $[\boldsymbol{\alpha}]_{D}^{22}-38^{\circ}\left(c 1.3, \mathrm{CHCl}_{3}\right) .^{2}$

\section{References:}

1. Greshock, T. J. \& Williams, R. M. Improved biomimetic total synthesis of d,1-stephacidin A. Org. Lett. 9, 4255-4258 (2007).

2. Steyn, P. S. The structures of five diketopiperazines from Aspergillus ustus. Tetrahedron 29, 107-120 (1973). 

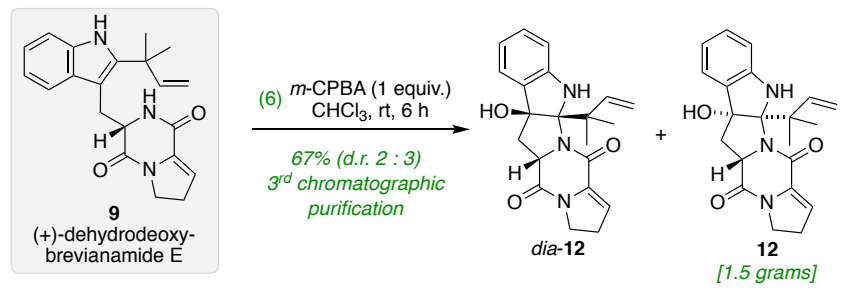

Step (6) - Inspired by the work of Kametani ${ }^{1}$ and Wolff. ${ }^{2}$ To a rapidly stirred solution of dehydrodeoxy-brevianamide $\mathrm{E}(9)(4.00 \mathrm{~g}, 11.5 \mathrm{mmol})$ in $\mathrm{CHCl}_{3}(80 \mathrm{~mL})$ at room temperature was added dropwise over $4 \mathrm{~h}$ a solution of $m$-CPBA in $\mathrm{CHCl}_{3}(0.29 \mathrm{M}, 40 \mathrm{~mL}, 11.7 \mathrm{mmol})$.* The reaction was stirred for $2 \mathrm{~h}$ at room temperature and quenched by the addition of saturated aq. $\mathrm{Na}_{2} \mathrm{~S}_{2} \mathrm{O}_{3}(20 \mathrm{~mL})$ and saturated aq. $\mathrm{NaHCO}_{3}(20 \mathrm{~mL})$. The reaction mixture was diluted with EtOAc $(200 \mathrm{~mL})$ and $\mathrm{CHCl}_{3}(150 \mathrm{~mL})$ and the phases separated. The aqueous phase was back-extracted with $\mathrm{CHCl}_{3}(50 \mathrm{~mL})$ and the combined organics washed with saturated aq. $\mathrm{NaHCO}_{3}$ $(80 \mathrm{~mL})$, brine $(80 \mathrm{~mL})$, dried $\left(\mathrm{Na}_{2} \mathrm{SO}_{4}\right)$ and concentrated under reduced pressure. Flash column chromatography (1.5:7:3 petroleum spirit/ $\mathrm{CH}_{2} \mathrm{Cl}_{2} / i-\mathrm{Pr}_{2} \mathrm{O}$ to $7: 3 \mathrm{CH}_{2} \mathrm{Cl}_{2} / i-\mathrm{Pr}_{2} \mathrm{O}$ to EtOAc to $i-\mathrm{PrOH})$ of the crude reaction product gave dehydro-brevianamide $\mathrm{E}$ (12) $(1.43 \mathrm{~g}, 3.91 \mathrm{mmol}$, $34 \%$ ) as a white foam, a fraction containing mixed dehydro-brevianamide $\mathrm{E}$ (12) and compound dia-12 (1.37 g, 18:82 12/dia-12, $3.75 \mathrm{mmol}, 33 \%$ ) as a cream foam, alongside recovered starting material dehydrodeoxy-brevianamide E (9) $(270 \mathrm{mg}, 0.774 \mathrm{mmol}, 7 \%)$ as a cream foam. Overall, 12/dia-12 (2.80 g, 60:40, $7.67 \mathrm{mmol}, 67 \%)$. A second round of chromatography on the mixed dehydro-brevianamide E (12) and compound dia-12 fraction gave additional pure dehydrobrevianamide E (12) $(0.108 \mathrm{~g}, 0.296 \mathrm{mmol}, 3 \%)$ as a cream glass and pure minor diastereomer dia-12 (0.454 g, $1.24 \mathrm{mmol}, 11 \%)$ as a white foam. At this point, further chromatography was deemed unnecessary since $92 \%$ of the major diastereomer dehydro-brevianamide E (12) had been isolated in pure form.

* $m$-CPBA ( $69 \% \mathrm{w} / \mathrm{w}$ by iodometric titration, $4.38 \mathrm{~g}, 17.5 \mathrm{mmol})$ was added to $\mathrm{CHCl}_{3}(60 \mathrm{~mL})$ and dried over $\mathrm{Na}_{2} \mathrm{SO}_{4}(27.5 \mathrm{~g})$ with stirring for 40 mins.

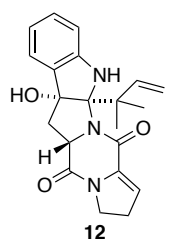

$\mathbf{R}_{\mathbf{f}} 0.15\left(7: 3 \mathrm{CH}_{2} \mathrm{Cl}_{2} / i-\mathrm{Pr}_{2} \mathrm{O}\right)$;

${ }^{1} \mathbf{H}$ NMR $\left(500 \mathrm{MHz}, \mathrm{CDCl}_{3}\right) \delta 7.24(\mathrm{dd}, J=7.5,1.2 \mathrm{~Hz}, 1 \mathrm{H}), 7.18($ app. td, $J=7.7,1.3 \mathrm{~Hz}, 1 \mathrm{H})$, 6.81 (td, $J=7.4,1.0 \mathrm{~Hz}, 1 \mathrm{H}), 6.74$ (app. dt, $J=7.9,0.8 \mathrm{~Hz}, 1 \mathrm{H}), 6.40-6.34$ (m, 2H), 6.16 (app. t, $J=3.1 \mathrm{~Hz}, 1 \mathrm{H}), 5.15(\mathrm{dd}, J=17.7,1.4 \mathrm{~Hz}, 1 \mathrm{H}), 5.07(\mathrm{dd}, J=10.9,1.3 \mathrm{~Hz}, 1 \mathrm{H}), 4.06(\mathrm{ddd}, J=$ 12.0, 8.7, 7.3 Hz, 1H), $3.93-3.83(\mathrm{~m}, 1 \mathrm{H}), 3.81(\mathrm{dd}, J=11.5,7.3 \mathrm{~Hz}, 1 \mathrm{H}), 2.79-2.69(\mathrm{~m}, 4 \mathrm{H})$, 2.43 (br. s, 1H), 1.34 (s, 3H), 1.27 (s, 3H) ppm;

${ }^{13}$ C NMR $\left(126 \mathrm{MHz}, \mathrm{CDCl}_{3}\right) \delta 163.7,162.1,149.4,144.7,136.4,131.0,129.8,123.8,120.6$, $120.2,113.4,111.2,91.5,89.3,60.3,45.8,45.0,36.7,29.0,27.6,23.2 \mathrm{ppm}$;

IR (film, cm cm $^{-1}$ 3362, 2967, 2926, 1670, 1634, 1609, 1485, 1468, 1437, 1393, 1360;

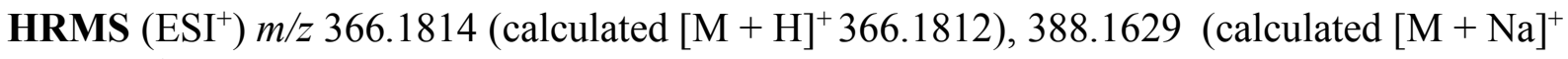
388.1632);

$[\boldsymbol{\alpha}]_{D}^{20.9}-208^{\circ}(c$ 0.23, EtOH). 


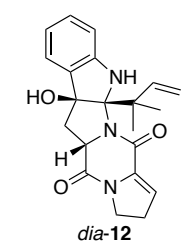

$\mathbf{R}_{\mathbf{f}} 0.09\left(7: 3 \mathrm{CH}_{2} \mathrm{Cl}_{2} / i-\mathrm{Pr}_{2} \mathrm{O}\right)$;

${ }^{1} \mathbf{H}$ NMR $\left(500 \mathrm{MHz}, \mathrm{CDCl}_{3}\right) \delta 7.22(\mathrm{~d}, J=7.5 \mathrm{~Hz}, 1 \mathrm{H}), 7.13$ (app. td, $\left.J=7.7,1.3 \mathrm{~Hz}, 1 \mathrm{H}\right), 6.76$ (app. td, $J=7.4,0.9 \mathrm{~Hz}, 1 \mathrm{H}$ ), 6.62 (app. dt, $J=7.9,0.8 \mathrm{~Hz}, 1 \mathrm{H}$ ), 6.47 (br. s, 1H), 6.34 (dd, $J=$ $17.7,10.8 \mathrm{~Hz}, 1 \mathrm{H}), 6.06$ (app. t, $J=3.0 \mathrm{~Hz}, 1 \mathrm{H}), 5.22$ (dd, $J=17.7,1.3 \mathrm{~Hz}, 1 \mathrm{H}), 5.10$ (dd, $J=$ $10.9,1.3 \mathrm{~Hz}, 1 \mathrm{H}), 4.73$ (app. t, $J=8.8 \mathrm{~Hz}, 1 \mathrm{H}), 4.00$ (ddd, $J=12.4,11.1,6.1 \mathrm{~Hz} 1 \mathrm{H}$ ), 3.82 (dddd, $J=12.3,11.4,8.3,0.8 \mathrm{~Hz}, 1 \mathrm{H}), 2.86(\mathrm{ddd}, J=13.6,8.6,0.7 \mathrm{~Hz}, 1 \mathrm{H}), 2.80-2.62(\mathrm{~m}, 3 \mathrm{H}), 2.57$ (br. s $1 \mathrm{H}), 1.45$ (s, 3H), 1.35 (s, 3H) ppm;

${ }^{13}$ C NMR $\left(126 \mathrm{MHz}, \mathrm{CDCl}_{3}\right) \delta 161.9,156.2,147.0,144.8,134.7,131.5,130.4,123.8,119.5$, $119.3,114.1,109.9,95.3,89.6,60.5,46.6,45.4,43.4,28.2,27.2,22.5$ ppm;

IR (film, cm cm $^{-1}$ 3360, 2965, 2924, 2854, 1665, 1632, 1611, 1487, 1466, 1439, 1414, 1371;

HRMS $\left(\mathrm{ESI}^{+}\right) \mathrm{m} / z$ 366.1807 (calculated $[\mathrm{M}+\mathrm{H}]^{+} 366.1812$ );

$[\boldsymbol{\alpha}]_{D}^{22.3}+86.3^{\circ}(c 0.19, \mathrm{EtOH})$.

\section{References:}

1. Kametani, T., Kanaya, N. \& Ihara, M. Asymmetric total synthesis of brevianamide E. J. Am. Chem. Soc. 1980, 102 (11), 3974-3975.

2. Adam, W., Bosio, S. G. \& Wolff, B. T. Chiral-Auxiliary-Controlled Diastereoselectivity in the Epoxidation of Enecarbamates with DMD and mCPBA. Org. Lett. 2003, 5 (6), 819-822. 

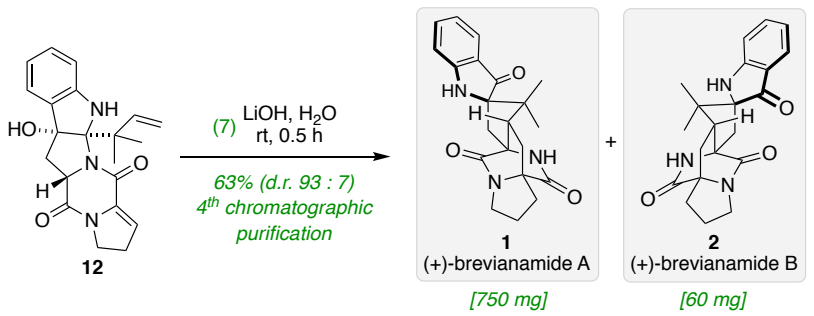

Step (7) - The synthesis of brevianamide A (1) was carried out under aerobic conditions, inspired by the work of Kishi ${ }^{1}$ and Williams. ${ }^{2}$ Effort was made to limit the exposure of the material to light once the reaction was complete, to prevent photolysis of brevianamide A (1) to brevianamide C and D. ${ }^{3}$ To a solution of dehydro-brevianamide E (12) $(1.30 \mathrm{~g}, 3.56 \mathrm{mmol})$ was added a $1 \mathrm{M}$ solution of aq. $\mathrm{LiOH}(450 \mathrm{~mL})$ and the reaction stirred rapidly, with vigorous manual shaking every 2-3 minutes to facilitate gradual dissolution of the starting material. After 30 minutes the reaction mixture was extracted with $\mathrm{CH}_{2} \mathrm{Cl}_{2}(2 \times 450 \mathrm{~mL}, 2 \times 200 \mathrm{~mL})$, and the combined organics dried $\left(\mathrm{Na}_{2} \mathrm{SO}_{4}\right)$. Flash column chromatography $\left(2: 8\right.$ to $3: 7$ to $1: 1 \mathrm{THF} / \mathrm{CHCl}_{3}$ to $25: 25: 1$ $\left.\mathrm{THF} / \mathrm{CHCl}_{3} / i-\mathrm{PrOH}\right)$ gave (+)-brevianamide A (1) $\left(905 \mathrm{mg}, 16.2 \% \mathrm{CHCl}_{3} \mathrm{w} / \mathrm{w}, 2.07 \mathrm{mmol}\right.$, $58 \%)^{*}$ as a yellow amorphous solid and (+)-brevianamide B (2) $(59.8 \mathrm{mg}, 0.164 \mathrm{mmol}, 5 \%)$ as a yellow crystalline solid. A sample of (+)-brevianamide A (1) was crystallised from $\mathrm{CHCl}_{3}$ by slow vapour diffusion with $\mathrm{Et}_{2} \mathrm{O}$. See pages 58-59 for X-ray crystal structure of brevianamide $\mathrm{A} \cdot \mathrm{CHCl}_{3}$.

*In Birch's original isolation paper brevianamide A 'crystallized from $\mathrm{CHCl}_{3}$ in needles containing one molecule of solvent of crystallisation'. ${ }^{4}$ The reported crystal structure for 5-bromo-brevianamide A also showed co-crystallisation with one molecule of acetone. ${ }^{5}$ Consistent with these observations, we found that a certain portion of solvent could not be removed from brevianamide A under high vacuum. Proteo-solvent was replaced with deutero-solvent to record NMR spectra for characterisation.

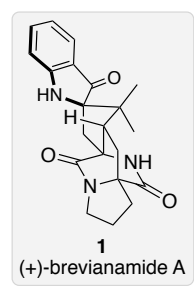

Spectroscopic data matched literature values reported by Zhu Weiming and co-workers. ${ }^{6}$ Subtle differences in ${ }^{1} \mathrm{H}$ NMR data are attributed to concentration effects in $\mathrm{CDCl}_{3}$ (see page 42).

$\mathbf{R}_{\mathbf{f}} 0.21\left(3: 7 \mathrm{THF} / \mathrm{CHCl}_{3}\right)$;

MP $173-179{ }^{\circ} \mathrm{C}$, literature: $175-180{ }^{\circ} \mathrm{C}$ ' with loss of solvent' ${ }^{4}$

${ }^{1}$ H NMR $\left(500 \mathrm{MHz}, \mathrm{CDCl}_{3}\right) \delta 7.57(\mathrm{dd}, J=7.7,1.2 \mathrm{~Hz}, 1 \mathrm{H}), 7.44(\mathrm{ddd}, J=8.3,7.1,1.4 \mathrm{~Hz}, 1 \mathrm{H})$, $6.84-6.78(\mathrm{~m}, 2 \mathrm{H}), 6.62$ (br. s, $1 \mathrm{H}), 4.98$ (br. s, $1 \mathrm{H}), 3.52-3.41(\mathrm{~m}, 2 \mathrm{H}), 2.81-2.74(\mathrm{~m}, 2 \mathrm{H})$, $2.40(\mathrm{ddd}, J=9.8,7.3,1.1 \mathrm{~Hz}, 1 \mathrm{H}), 2.35$ (d, $J=15.6 \mathrm{~Hz}, 1 \mathrm{H}), 2.04$ (app. pd, $J=6.8,1.6 \mathrm{~Hz}, 2 \mathrm{H})$, $1.96-1.81(\mathrm{~m}, 3 \mathrm{H}), 1.12(\mathrm{~s}, 3 \mathrm{H}), 0.93(\mathrm{~s}, 3 \mathrm{H}) \mathrm{ppm}$;

${ }^{13}$ C NMR $\left(126 \mathrm{MHz}, \mathrm{CDCl}_{3}\right) \delta 202.2,172.4,169.8,160.2,137.8,124.8,121.2,119.3,112.1,78.9$, 69.5, 67.8, 55.7, 48.4, 44.1, 37.4, 29.2, 29.0, 25.1, 24.1, 19.9 ppm;

${ }^{1}$ H NMR $\left(500 \mathrm{MHz},\left(\mathrm{CD}_{3}\right)_{2} \mathrm{SO}\right) \delta 8.65$ (br. s, $\left.1 \mathrm{H}\right), 7.84$ (br. s, $\left.1 \mathrm{H}\right), 7.41$ (ddd, $J=8.3,7.0,1.4 \mathrm{~Hz}$, $1 \mathrm{H}), 7.37(\mathrm{~d}, J=7.7 \mathrm{~Hz}, 1 \mathrm{H}), 6.81(\mathrm{dd}, J=8.2,0.8 \mathrm{~Hz}, 1 \mathrm{H}), 6.64(\mathrm{ddd}, J=7.8,7.0,0.8 \mathrm{~Hz}, 1 \mathrm{H})$, $3.34-3.23(\mathrm{~m}, 2 \mathrm{H}), 2.55$ (ddd, $J=10.1,7.5,1.1 \mathrm{~Hz}, 1 \mathrm{H}), 2.49-2.44(\mathrm{~m}, 2 \mathrm{H}), 2.39(\mathrm{~d}, J=15.1$ $\mathrm{Hz}, 1 \mathrm{H}), 2.01-1.91(\mathrm{~m}, 1 \mathrm{H}), 1.90-1.77(\mathrm{~m}, 3 \mathrm{H}), 1.68(\mathrm{dd}, J=13.1,7.6 \mathrm{~Hz}, 1 \mathrm{H}), 0.98(\mathrm{~s}, 3 \mathrm{H})$, 0.68 (s, 3H) ppm; 
${ }^{13}$ C NMR (126 MHz, $\left.\left(\mathrm{CD}_{3}\right)_{2} \mathrm{SO}\right) \delta$ 199.9, 172.1, 169.5, 160.5, 137.2, 123.6, 119.4, 116.7, 111.1, 79.0, 68.5, 66.4, 53.1, 48.3, 43.2, 39.5, 28.4, 27.2, 24.6, 21.2, 17.7 ppm;

IR (film, cm ${ }^{-1}$ ) 3314, 3229, 2938, 2876, 1667, 1618, 1491, 1466, 1395;

HRMS $\left(\mathrm{ESI}^{+}\right) \mathrm{m} / z$ 366.1818 (calculated $[\mathrm{M}+\mathrm{H}]^{+} 366.1812$ );

$[\boldsymbol{\alpha}]_{D}^{\mathbf{2 3 . 5}}+316^{\circ}(c$ 0.12, EtOH $)$, literature: $[\boldsymbol{\alpha}]_{\boldsymbol{D}}^{\mathbf{2 5}}+413^{\circ}(\mathrm{EtOH}) \dot{4}^{4}$

e.r. 93:7, after crystallisation 99:1 (Chiralpak IA, 1:1 $i$-PrOH/hexane, $1 \mathrm{~mL} \mathrm{~min}^{-1}, \lambda 254 \mathrm{~nm}$ ) $t_{\text {Rminor }}$ $=5.41 \mathrm{~min}, \mathrm{t}_{\mathrm{Rmajor}}=6.72 \mathrm{~min}$. See Pages 54-55 for chiral HPLC traces.

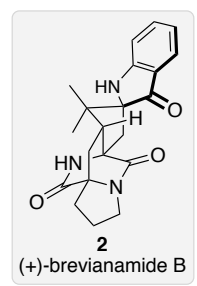

Brevianamide $\mathrm{B}$ (2) was only sparingly soluble in $\mathrm{CDCl}_{3}$, consistent with the observation in Birch's original isolation paper that brevianamide B 'was insoluble in most solvents except hot DMSO and $\mathrm{CF}_{3} \mathrm{CO}_{2} \mathrm{H}$, the latter causing decomposition'. ${ }^{3}{ }^{1} \mathrm{H}$ and ${ }^{13} \mathrm{C}$ NMR spectra could be recorded using a saturated sample in $\mathrm{CDCl}_{3}$, to enable comparison to literature data, ${ }^{7,8}$ however 2D NMR spectra were unobtainable. High quality 1D and 2D NMR spectra were instead recorded in $\left(\mathrm{CD}_{3}\right)_{2} \mathrm{SO}$.

$\mathbf{R}_{\mathbf{f}} 0.08\left(3: 7 \mathrm{THF} / \mathrm{CHCl}_{3}\right)$;

MP 286-290 ${ }^{\circ} \mathrm{C}$ (decomposition), literature: $324-328^{\circ} \mathrm{C}$ (decomposition); ${ }^{3}$

${ }^{1} \mathbf{H}$ NMR (500 MHz, $\mathrm{CDCl}_{3}$ ) $\delta 7.56$ (app. ddt, $\left.J=7.6,1.3,0.7 \mathrm{~Hz}, 1 \mathrm{H}\right), 7.43$ (ddd, $J=8.4,7.1,1.4$ $\mathrm{Hz}, 1 \mathrm{H}), 6.84-6.78$ (m, 2H), 5.78 (br. s, 1H), 4.73 (br. s, 1H), $3.50-3.44$ (m, 2H), 3.31 (ddd, $J$ $=10.3,7.4,1.3 \mathrm{~Hz}, 1 \mathrm{H}), 3.27(\mathrm{~d}, J=15.6 \mathrm{~Hz}, 1 \mathrm{H}), 2.74(\mathrm{ddd}, J=13.1,7.0,6.1 \mathrm{~Hz}, 1 \mathrm{H}), 2.06-$ $1.93(\mathrm{~m}, 3 \mathrm{H}), 1.90-1.77(\mathrm{~m}, 2 \mathrm{H}), 1.70(\mathrm{~d}, J=15.7,1 \mathrm{H}), 1.14(\mathrm{~s}, 3 \mathrm{H}), 0.83(\mathrm{~s}, 3 \mathrm{H}) \mathrm{ppm}$;

${ }^{13}$ C NMR $\left(126 \mathrm{MHz}, \mathrm{CDCl}_{3}\right) \delta 203.9,173.4,169.2,160.3,137.5,125.2,120.1,119.2,111.5,77.7$, 68.9, 66.5, 49.7, 46.6, 44.0, 36.8, 29.3, 28.6, 25.1, 22.6, 20.5 ppm;

${ }^{1} \mathbf{H}$ NMR $\left(500 \mathrm{MHz},\left(\mathrm{CD}_{3}\right)_{2} \mathrm{SO}\right) \delta 8.64$ (br. s, $\left.1 \mathrm{H}\right), 7.41$ (ddd, $\left.J=8.3,7.1,1.4 \mathrm{~Hz}, 1 \mathrm{H}\right), 7.33(\mathrm{~d}, J$ $=7.7 \mathrm{~Hz}, 1 \mathrm{H}), 7.27$ (br. s, $1 \mathrm{H}), 6.89$ (app. dt, $J=8.3,0.9 \mathrm{~Hz}, 1 \mathrm{H}), 6.64$ (ddd, $J=7.8,7.0,0.9 \mathrm{~Hz}$, $1 \mathrm{H}), 3.35-3.24(\mathrm{~m}, 2 \mathrm{H}), 3.01(\mathrm{ddd}, J=10.3,7.5,1.1 \mathrm{~Hz}, 1 \mathrm{H}), 2.67(\mathrm{~d}, J=15.1 \mathrm{~Hz}, 1 \mathrm{H}), 2.46$ $(\mathrm{dd}, J=11.9,6.1 \mathrm{~Hz}, 1 \mathrm{H}), 2.02-1.90(\mathrm{~m}, 3 \mathrm{H}), 1.85-1.74(\mathrm{~m}, 2 \mathrm{H}), 1.63(\mathrm{dd}, J=13.0,7.5 \mathrm{~Hz}$, $1 \mathrm{H}), 1.05(\mathrm{~s}, 3 \mathrm{H}), 0.63(\mathrm{~s}, 3 \mathrm{H}) \mathrm{ppm}$;

${ }^{13}$ C NMR (126 MHz, $\left.\left(\mathrm{CD}_{3}\right)_{2} \mathrm{SO}\right) \delta$ 204.7, 172.6, 169.2, 161.3, 137.2, 123.8, 118.1, 116.9, 111.4, 77.5, 68.1, 65.2, 48.8, 46.0, 43.3, 33.4, 28.4, 27.5, 24.4, 21.9, $19.7 \mathrm{ppm}$;

IR (film, cm ${ }^{-1}$ ) 3348, 3231, 2949, 2878, 1705, 1664, 1653, 1616, 1587, 1495, 1468;

HRMS $\left(\mathrm{ESI}^{+}\right) \mathrm{m} / \mathrm{z} 366.1796$ (calculated $[\mathrm{M}+\mathrm{H}]^{+} 366.1812$ );

$[\boldsymbol{\alpha}]_{\boldsymbol{D}}^{\mathbf{2 4 . 7}}+136^{\circ}\left(c 0.23,2.5 \% \mathrm{HCO}_{2} \mathrm{H}\right.$ in $\left.\mathrm{CH}_{2} \mathrm{Cl}_{2}\right)$, literature (enantiomer): $[\boldsymbol{\alpha}]_{\boldsymbol{D}}^{\mathbf{2 5}}-147^{\circ}(c 0.23,2.5 \%$ $\mathrm{HCO}_{2} \mathrm{H}$ in $\left.\mathrm{CH}_{2} \mathrm{Cl}_{2}\right),{ }^{8}[\boldsymbol{\alpha}]_{\boldsymbol{D}}^{25}-124^{\circ}\left(c 0.81,2.5 \% \mathrm{HCO}_{2} \mathrm{H}\right.$ in $\left.\mathrm{CH}_{2} \mathrm{Cl}_{2}\right){ }^{7}$

e.r. $93: 7$ (Chiralpak IA, 1:3 EtOH/hexane, $\left.1 \mathrm{~mL} \mathrm{m^{-1 }}, \lambda 254 \mathrm{~nm}\right) t_{\mathrm{Rminor}}=13.99 \mathrm{~min}, \mathrm{t}_{\mathrm{Rmajor}}=$ 26.10 min. See Pages 56-57 for chiral HPLC traces.

\section{References:}

1. Hutchison, A. J. \& Kishi, Y. Stereospecific total synthesis of dl-austamide. J. Am. Chem. Soc. 101, 6786-6788 (1979).

2. Greshock, T. J. \& Williams, R. M. Improved biomimetic total synthesis of d,l- stephacidin A. Org. Lett. 9, 4255-4258 (2007). 
3. Birch, A. J. \& Russell, R. A. Studies in relation to biosynthesis-XLIV: Structural elucidations of brevianamides-B, -C, -D and -F. Tetrahedron 28, 2999-3008 (1972).

4. Birch, A. J. \& Wright, J. J. Studies in relation to biosynthesis-XLII: The structural elucidation and some aspects of the biosynthesis of the brevianamides-A and -E. Tetrahedron 26, 2329-2344 (1970).

5. Coetzer, J. The structure and absolute configuration of 5-bromobrevianamide A. Acta Crystallographica Section B 30, 2254-2256 (1974).

6. Lingling, C., Tonghan, Z., Guoliang, Z., Yunlong, L., Cong, W., Pawinee, P., Arthit, C. \& Weiming, Z. Bioactive Natural Products from the Marine-Derived Penicillium brevicompactum OUCMDZ-4920. Chin. J. Org. Chem. 37, 2752-2762 (2017).

7. Williams, R. M., Glinka, T., Kwast, E., Coffman, H. \& Stille, J. K. Asymmetric, stereocontrolled total synthesis of (-)-brevianamide B. J. Am. Chem. Soc. 112, 808-821 (1990).

8. Frebault, F. C. \& Simpkins, N. S. A cationic cyclisation route to prenylated indole alkaloids: synthesis of malbrancheamide B and brevianamide B, and progress towards stephacidin A. Tetrahedron 66, 6585-6596 (2010). 

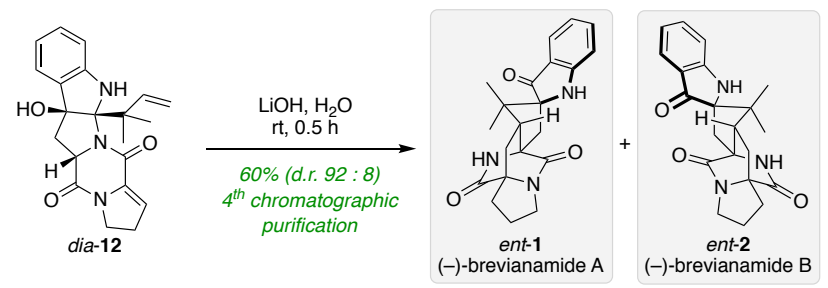

To a solution of compound dia-12 $(234 \mathrm{mg}, 0.640 \mathrm{mmol})$ was added a $1 \mathrm{M}$ solution of aq. LiOH $(80 \mathrm{~mL})$ and the reaction stirred rapidly, with vigorous manual shaking every $2-3$ minutes to facilitate gradual dissolution of the starting material. After 30 minutes the reaction mixture was extracted with $\mathrm{CH}_{2} \mathrm{Cl}_{2}(4 \times 100 \mathrm{~mL})$, and the combined organics dried $\left(\mathrm{Na}_{2} \mathrm{SO}_{4}\right)$. Flash column chromatography (2:8 to $3: 7$ to $1: 1 \mathrm{THF} / \mathrm{CHCl}_{3}$ to $\left.25: 25: 1 \mathrm{THF} / \mathrm{CHCl}_{3} / i-\mathrm{PrOH}\right)$ gave (-)brevianamide A (ent-1) $\left(155 \mathrm{mg}, 17.5 \% \mathrm{CHCl}_{3} \mathrm{w} / \mathrm{w}, 0.350 \mathrm{mmol}, 55 \%\right)$ * as a yellow amorphous solid and (-)-brevianamide B (ent-2) (12 mg, $0.032 \mathrm{mmol}, 5 \%)$ as a yellow crystalline solid.

*In Birch's original isolation paper brevianamide A 'crystallized from $\mathrm{CHCl}_{3}$ in needles

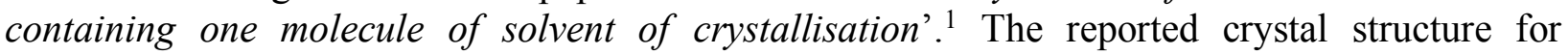
5-bromo-brevianamide A also showed co-crystallisation with one molecule of acetone. ${ }^{2}$ Consistent with these observations, we found that a certain portion of solvent could not be removed from brevianamide A under high vacuum. Proteo-solvent was replaced with deutero-solvent to record NMR spectra for characterisation.

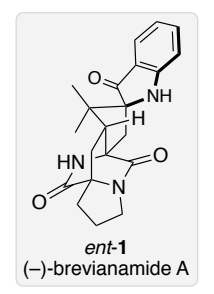

$[\boldsymbol{\alpha}]_{\boldsymbol{D}}^{\mathbf{2 4 . 7}}-281^{\circ}(c 0.12, \mathrm{EtOH})$, literature (enantiomer): $[\boldsymbol{\alpha}]_{\boldsymbol{D}}^{\mathbf{2 5}}+413^{\circ}(\mathrm{EtOH}) \dot{1}^{1}$

e.r. 95:5 (Chiralpak IA, 1:1 $i$-PrOH/hexane, $\left.1 \mathrm{~mL} \mathrm{~min}^{-1}, \lambda 254 \mathrm{~nm}\right) t_{\mathrm{Rmajor}}=5.38 \mathrm{~min}, \mathrm{t}_{\mathrm{Rminor}}=$ 6.74 min. See Pages 54-55 for chiral HPLC traces.

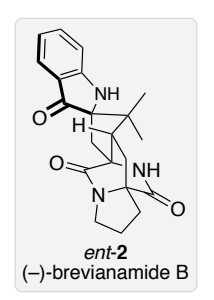

$[\boldsymbol{\alpha}]_{\boldsymbol{D}}^{\mathbf{2 5 . 1}}-119^{\circ}\left(c 0.23,2.5 \% \mathrm{HCO}_{2} \mathrm{H}\right.$ in $\left.\mathrm{CH}_{2} \mathrm{Cl}_{2}\right)$, literature: $[\boldsymbol{\alpha}]_{D}^{25}-147^{\circ}\left(c 0.23,2.5 \% \mathrm{HCO}_{2} \mathrm{H}\right.$ in $\left.\mathrm{CH}_{2} \mathrm{Cl}_{2}\right),{ }^{3}[\boldsymbol{\alpha}]_{\boldsymbol{D}}^{25}-124^{\circ}$ (c $0.81,2.5 \% \mathrm{HCO}_{2} \mathrm{H}$ in $\left.\mathrm{CH}_{2} \mathrm{Cl}_{2}\right),^{4}$

e.r. 92:8 (Chiralpak IA, 1:3 EtOH/hexane, $\left.1 \mathrm{~mL} \mathrm{~min}^{-1}, \lambda 254 \mathrm{~nm}\right) \mathrm{t}_{\text {Rmajor }}=13.86 \mathrm{~min}, \mathrm{t}_{\mathrm{Rminor}}=$ 26.14 min. See Pages 56-57 for chiral HPLC traces.

\section{References:}

1. Birch, A. J., \& Wright, J. J. Studies in relation to biosynthesis - XLII: The structural elucidation and some aspects of the biosynthesis of the brevianamides-A and -E. Tetrahedron 1970, 26 (10), 2329-2344.

2. Coetzer, J. The structure and absolute configuration of 5-bromobrevianamide A. Acta Crystallographica Section B 30, 2254-2256 (1974). 
3. Frebault, F. C. \& Simpkins, N. S. A cationic cyclisation route to prenylated indole alkaloids: synthesis of malbrancheamide $\mathrm{B}$ and brevianamide $\mathrm{B}$, and progress towards stephacidin A. Tetrahedron 66, 6585-6596 (2010).

4. Williams, R. M., Glinka, T., Kwast, E., Coffman, H. \& Stille, J. K. Asymmetric, stereocontrolled total synthesis of (-)-brevianamide B. J. Am. Chem. Soc. 112, 808-821 (1990). 
3.10 Experimental Procedure for Compound 18

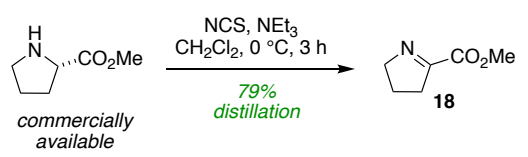

Compound 18 was prepared under anhydrous conditions, based on a procedure reported by Schmalz and co-workers. ${ }^{1}$ To a rapidly stirred suspension of L-proline methyl ester hydrochloride $(25.0 \mathrm{~g}, 151 \mathrm{mmol})$ and $\mathrm{NEt}_{3}(48.3 \mathrm{~mL}, 347 \mathrm{mmol})$ in $\mathrm{CH}_{2} \mathrm{Cl}_{2}(450 \mathrm{~mL})$ at $0{ }^{\circ} \mathrm{C}$ was added slowly, portion-wise, solid NCS $(22.2 \mathrm{~g}, 166 \mathrm{mmol})$. The mixture was stirred at room temperature for $3 \mathrm{~h}$ and then diluted with $\mathrm{Et}_{2} \mathrm{O}(350 \mathrm{~mL})$. A mixture of saturated aq. $\mathrm{NH}_{4} \mathrm{Cl}(150 \mathrm{~mL})$ and water $(150$ $\mathrm{mL}$ ) was added, the organic phase was separated and the aqueous phase was back-extracted with $\mathrm{CH}_{2} \mathrm{Cl}_{2}(2 \times 150 \mathrm{~mL})$. The combined organics were washed with a mixture of saturated aq. $\mathrm{NH}_{4} \mathrm{Cl}$ $(100 \mathrm{~mL})$ and water $(100 \mathrm{~mL})$, saturated aq. $\mathrm{NaHCO}_{3}(150 \mathrm{~mL})$, brine $(150 \mathrm{~mL})$, dried $\left(\mathrm{Na}_{2} \mathrm{SO}_{4}\right)$ and concentrated under reduced pressure. Vacuum distillation under reduced pressure (5 mbar) gave imine 18 (15.1 g, $119 \mathrm{mmol}, 79 \%)$ as a pale-yellow liquid. All spectroscopic data matched literature values. ${ }^{2}$<smiles>CC(=O)N1CCCC1</smiles>

BP $60-64{ }^{\circ} \mathrm{C}(5 \mathrm{mbar})$;

${ }^{1} \mathbf{H}$ NMR $\left(500 \mathrm{MHz}, \mathrm{CDCl}_{3}\right) \delta 4.04$ (app. tt, $\left.J=7.6,2.6 \mathrm{~Hz}, 2 \mathrm{H}\right), 3.80$ (s, 3H), 2.76 (app. ddt, $J=$ 8.5, 7.6, $2.6 \mathrm{~Hz}, 2 \mathrm{H}), 1.96-1.89(\mathrm{~m}, 2 \mathrm{H}) \mathrm{ppm}$;

${ }^{13} \mathbf{C}$ NMR $\left(126 \mathrm{MHz}, \mathrm{CDCl}_{3}\right) \delta 168.2,163.2,62.5,52.5,35.3,22.1 \mathrm{ppm}$.

\section{References:}

1. Huy, P., Neudörfl, J.-M. \& Schmalz, H.-G. A practical synthesis of trans-3- substituted proline derivatives through 1,4-addition. Org. Lett. 13, 216-219 (2011).

2. Sezen, B. \& Sames, D. Oxidative C-Arylation of Free (NH)-Heterocycles via Direct $\left(\mathrm{sp}^{3}\right) \mathrm{C}-\mathrm{H}$ Bond Functionalization. J. Am. Chem. Soc. 126, 13244-13246 (2004). 
3.11 Experimental Procedure for $B$-Prenyl-9-BBN

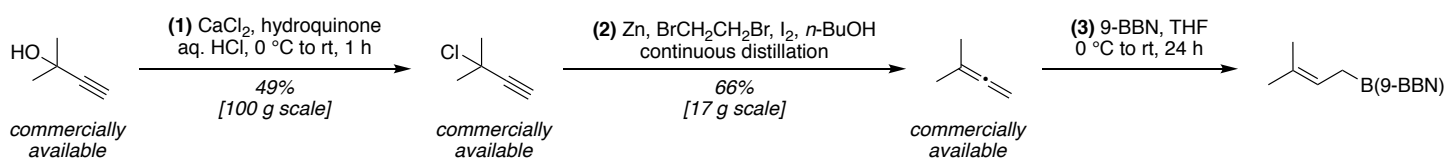

1,1-Dimethylallene is a commercially available reagent, but was prepared on large scale based on a procedure reported by Pfeffer and co-workers. ${ }^{1}$

Step (1) - Anhydrous $\mathrm{CaCl}_{2}(229 \mathrm{~g}, 2.06 \mathrm{~mol})$ was added in portions to a stirred mixture of concentrated hydrochloric acid $(800 \mathrm{~mL})$, 2-methyl-3-butyn-2-ol $(200 \mathrm{~mL}, 2.06 \mathrm{~mol})$ and hydroquinone $(1.82 \mathrm{~g}, 16.5 \mathrm{mmol})$ at $0{ }^{\circ} \mathrm{C}$ over 7 minutes and the reaction was stirred for $1 \mathrm{~h}$ at room temperature. The layers were seperated and solid $\mathrm{K}_{2} \mathrm{CO}_{3}$ added cautiously to the organic phase until effervescence ceased. The organic phase was then distilled under reduced pressure, collected up to $50{ }^{\circ} \mathrm{C}$ at 147 mbar. The crude distilate was redistilled under reduced pressure (147 mbar) to give 3-chloro-3-methyl-1-butyne (103 g, $1.00 \mathrm{~mol}, 49 \%)$ as a colourless oil. All spectroscopic data matched a comercially available sample.<smiles>[SiH3]C12CC1C2</smiles>

BP $32-36{ }^{\circ} \mathrm{C}(147 \mathrm{mbar})$;

${ }^{1} \mathbf{H}$ NMR $\left(500 \mathrm{MHz}, \mathrm{CDCl}_{3}\right) \delta 2.62(\mathrm{~s}, 1 \mathrm{H}), 1.87$ (s, 6H) ppm;

${ }^{13} \mathbf{C}$ NMR $\left(101 \mathrm{MHz}, \mathrm{CDCl}_{3}\right) \delta 86.7,72.0,57.1,34.7 \mathrm{ppm}$.

Step (2) - Zinc powder $(52.0 \mathrm{~g}, 760 \mathrm{mmol})$ was washed with $3 \%$ aq. $\mathrm{HCl}(4 \times 40 \mathrm{~mL})$, $2 \%$ aq. $\mathrm{CuSO}_{4}(2 \times 60 \mathrm{~mL})$, ethanol $(2 \times 60 \mathrm{~mL})$ and $n$-butanol $(2 \times 60 \mathrm{~mL})$. The treated zinc was suspended in $n$-butanol $(110 \mathrm{~mL})$ in a 3 neck RBF with a dropping funnel, magnetic stirrer bar and a distillation head fitted with a $30 \mathrm{~cm}$ Vigreux column. 3-Chloro-3-methyl-1-butyne $(44.4 \mathrm{~mL}$, $390 \mathrm{mmol})$ was placed in the dropping funnel along with 1,2-dibromoethane $(2.50 \mathrm{~mL}$, $29.0 \mathrm{mmol})$. A portion of the alkyne mixture $(3 \mathrm{~mL})$ was added to the zinc butanol slurry along with a few crystals of iodine and 1,2-dibromoethane $(2.50 \mathrm{~mL}, 29.0 \mathrm{mmol})$. The mixture was then heated cautiously (using a heat gun) with stirring until the reaction had clearly commenced. Slow addition of the remaining alkyne mixture followed, with external heating applied to maintain the reaction. The crude product was collected by distillation from the reaction mixture during the course of the reaction, and external heat applied to continue collection up to a temperature of 44 ${ }^{\circ} \mathrm{C}$. The crude distillate was redistilled to give 1,1-dimethylallene $(17.5 \mathrm{~g}, 257 \mathrm{mmol}, 66 \%)$ as a colourless liquid. All spectroscopic data matched literature values. ${ }^{1}$<smiles>C=C(C)C</smiles>

BP $40-42{ }^{\circ} \mathrm{C}$;

${ }^{1} \mathbf{H}$ NMR $\left(500 \mathrm{MHz}, \mathrm{CDCl}_{3}\right) \delta 4.52$ (hept, $\left.J=3.1 \mathrm{~Hz}, 2 \mathrm{H}\right), 1.69(\mathrm{t}, J=3.2 \mathrm{~Hz}, 6 \mathrm{H}) \mathrm{ppm}$;

${ }^{13} \mathbf{C}$ NMR $\left(151 \mathrm{MHz}, \mathrm{CDCl}_{3}\right) \delta 206.8,94.2,72.7,20.3$ ppm.

Step (3) - A solution of $B$-Prenyl-9-BBN was prepared under anhydrous conditions, based on a procedure reported by Trauner and co-workers. ${ }^{66}$ A solution of $9-\mathrm{BBN}$ in THF $(360 \mathrm{~mL}$, $179 \mathrm{mmol}, 0.5 \mathrm{M}$ in THF) was cooled to $0{ }^{\circ} \mathrm{C}$. Once the solution was observed to go cloudy, 1,1dimethylallene $(20.5 \mathrm{~mL}, 208 \mathrm{mmol})$ was added dropwise over 30 minutes with stirring. The reaction was warmed to room temperature and stirred for $18 \mathrm{~h}$ before being used directly in the reverse prenylation procedure described on page 15 . 


\section{References:}

1. Chengebroyen, J., Linke, M., Robitzer, M., Sirlin, C. \& Pfeffer, M. Palladium-mediated intramolecular $\mathrm{C}-\mathrm{N}$ bond formation involving allyl substituted pyridines. Application to a novel strategy for the synthesis of the skeleton of berberinium derivatives. J. Organomet. Chem. 687, 313-321 (2003).

2. Kuttruff, C. A., Zipse, H. \& Trauner, D. Concise Total Syntheses of Variecolortides A and B through an Unusual Hetero-Diels-Alder Reaction. Angew. Chem. Int. Ed. 50, 1402-1405 (2011). 
3.12 Experimental Procedure for $t$ - $\mathrm{BuOCl}$

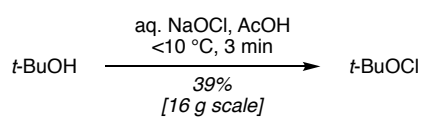

$t$ - $\mathrm{BuOCl}$ was prepared based on a procedure reported by Mintz and co-workers. ${ }^{1}$ For safety reasons exposure of the reaction and product to light was minimised.

An aqueous solution of bleach $(500 \mathrm{~mL}, 0.767 \mathrm{M}, 384 \mathrm{mmol})^{*}$ was cooled below $10{ }^{\circ} \mathrm{C}$ with stirring. A solution of $t$-BuOH $(43.3 \mathrm{~mL}, 383 \mathrm{mmol})$ and glacial acetic acid $(24.1 \mathrm{~mL}, 421 \mathrm{mmol})$ was added in a single portion to the rapidly stirred bleach and the stirring continued for $3 \mathrm{~min}$. The entire reaction mixture was transferred to a separating funnel, the aqueous layer discarded, the yellow organic layer washed with $10 \%$ aq. $\mathrm{Na}_{2} \mathrm{CO}_{3}(50 \mathrm{~mL})$ and water $(50 \mathrm{~mL})$. The product was dried $\left(\mathrm{CaCl}_{2}\right.$ approximately $\left.3 \mathrm{~g}\right)$ and decanted into its final container via pipette. The $t$-BuOCl (16.4 g, $151 \mathrm{mmol}, 39 \%$ ) was stored in a fridge over $\mathrm{CaCl}_{2}$ in an amber glass bottle as a yellow liquid.

*Bleach solution was titrated to a concentration of $0.767 \mathrm{M}$ by iodometric titration using sodium thiosulfate prior to use.

${ }^{1} \mathbf{H}$ NMR $\left(500 \mathrm{MHz}, \mathrm{CDCl}_{3}\right) \delta 1.33(\mathrm{~s}, 9 \mathrm{H}) \mathrm{ppm}$;

${ }^{13} \mathbf{C ~ N M R}\left(126 \mathrm{MHz}, \mathrm{CDCl}_{3}\right) \delta 84.1,27.0 \mathrm{ppm}$.

\section{References:}

1. Mintz, M. J. \& Walling, C. $t$-BUTYL HYPOCHLORITE. Org. Synth. 49, 9 (1969). 
4.1 ${ }^{1} \mathrm{H}$ NMR Spectrum of Compound $14\left(500 \mathrm{MHz}, \mathrm{CDCl}_{3}\right)$
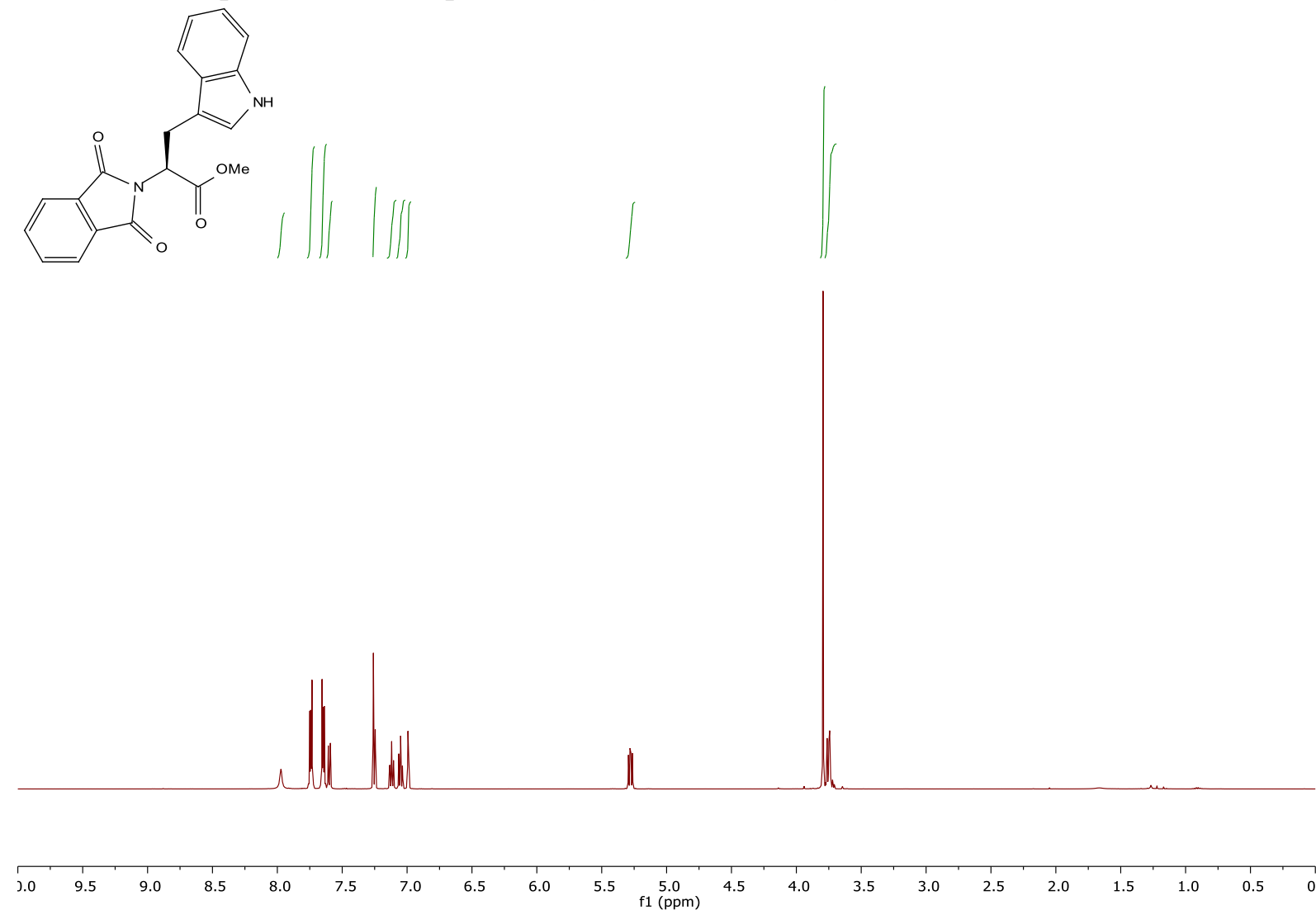

4.2 ${ }^{13} \mathrm{C}$ NMR Spectrum of Compound $14\left(126 \mathrm{MHz}, \mathrm{CDCl}_{3}\right)$
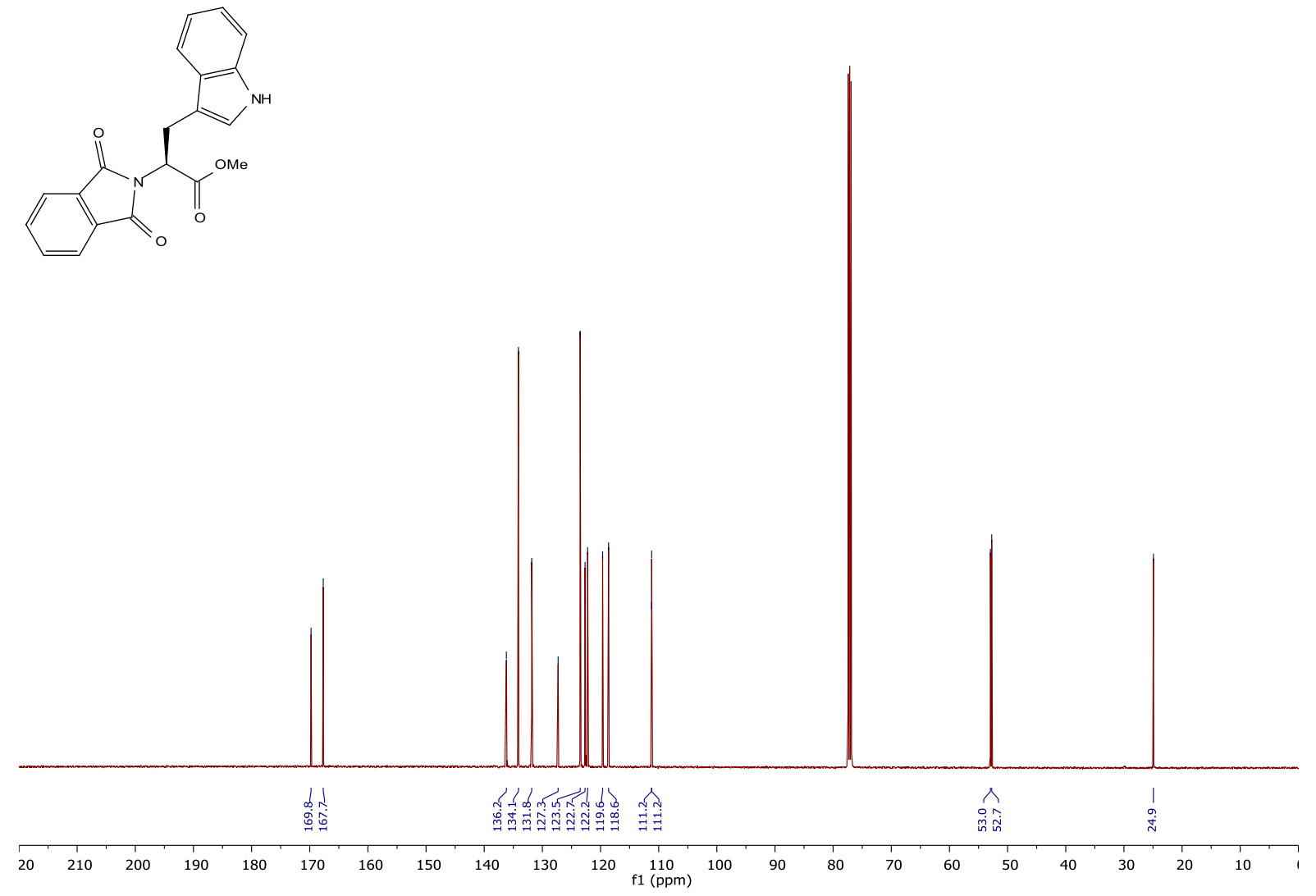
4.3 ${ }^{1} \mathrm{H}$ NMR Spectrum of Compound $15\left(600 \mathrm{MHz}, \mathrm{CDCl}_{3}\right)$
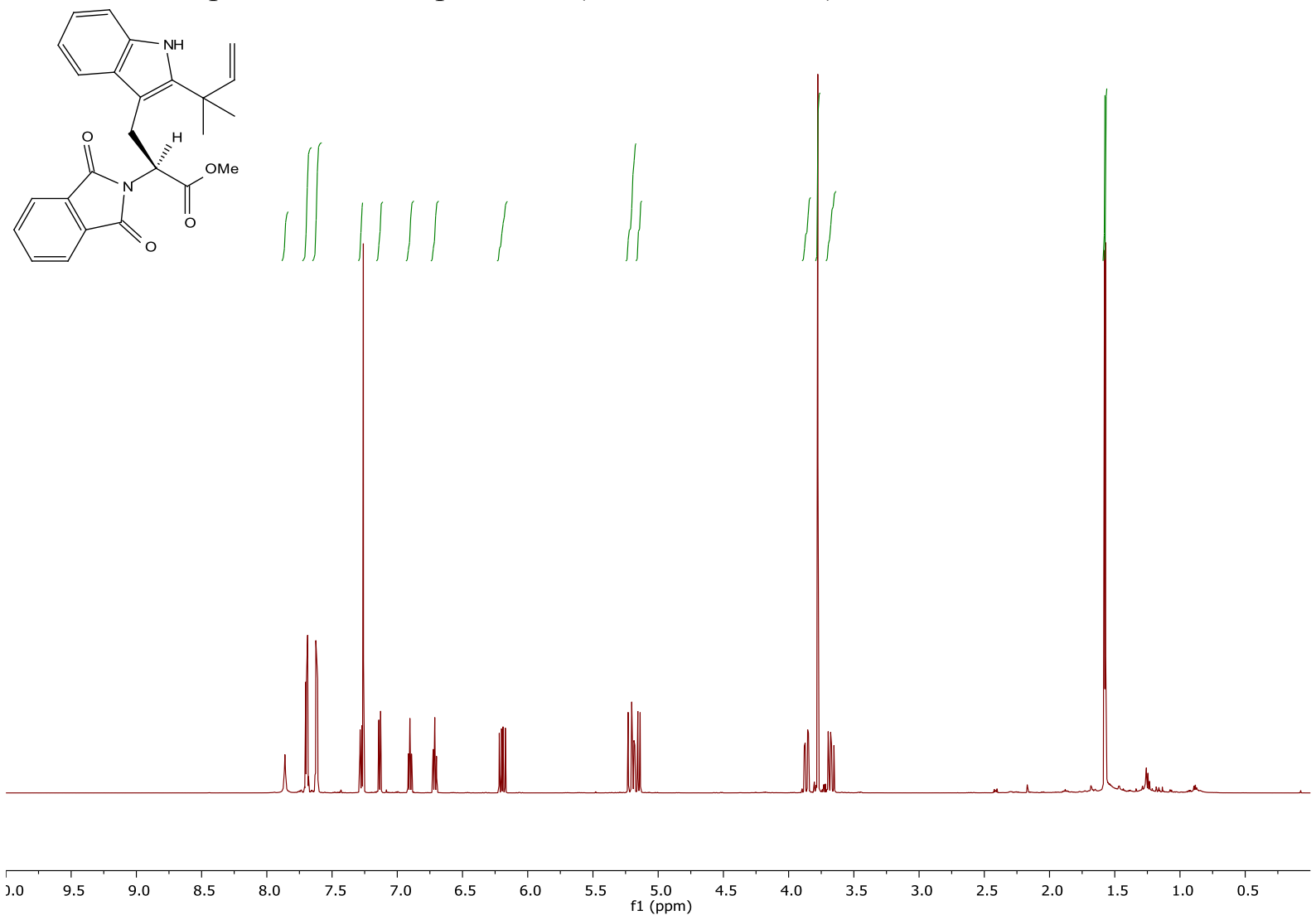

4.4 ${ }^{13} \mathrm{C}$ NMR Spectrum of Compound $15\left(151 \mathrm{MHz}, \mathrm{CDCl}_{3}\right)$
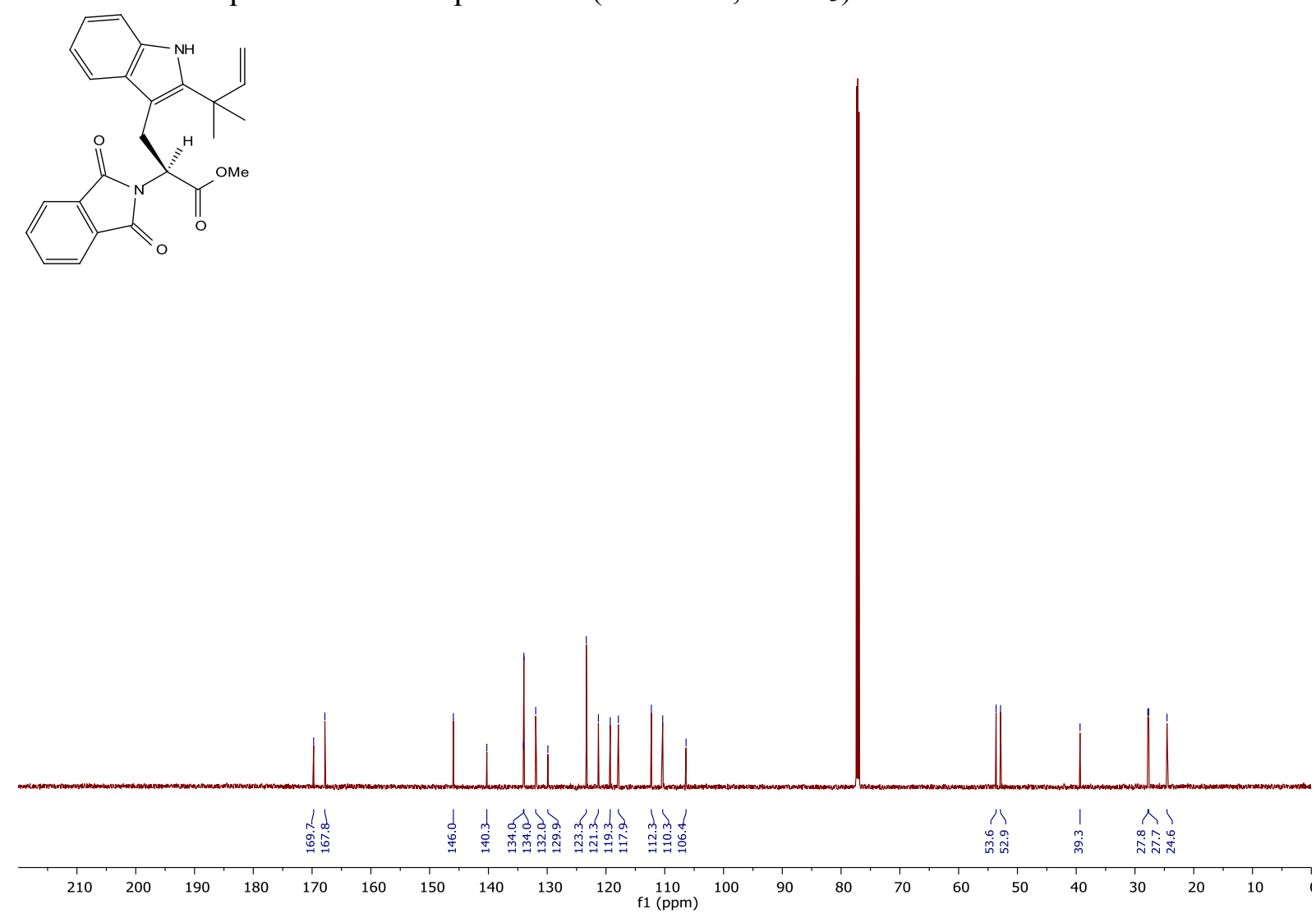
4.5 ${ }^{1} \mathrm{H}$ NMR Spectrum of Compound 16 (500 MHz, $\mathrm{CD}_{3} \mathrm{OD}$ )
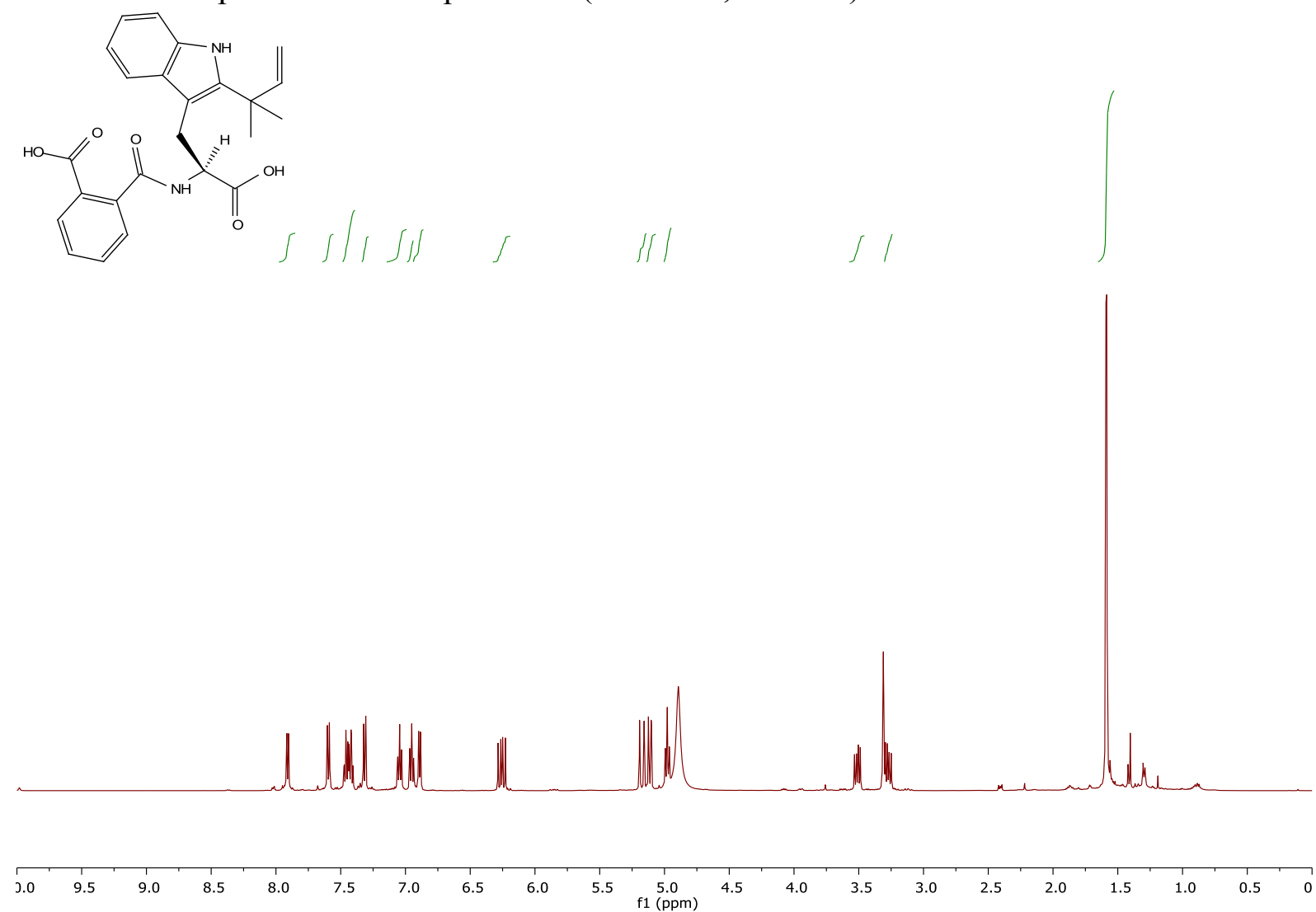

4.6 ${ }^{13} \mathrm{C}$ NMR Spectrum of Compound $16\left(126 \mathrm{MHz}, \mathrm{CD}_{3} \mathrm{OD}\right)$

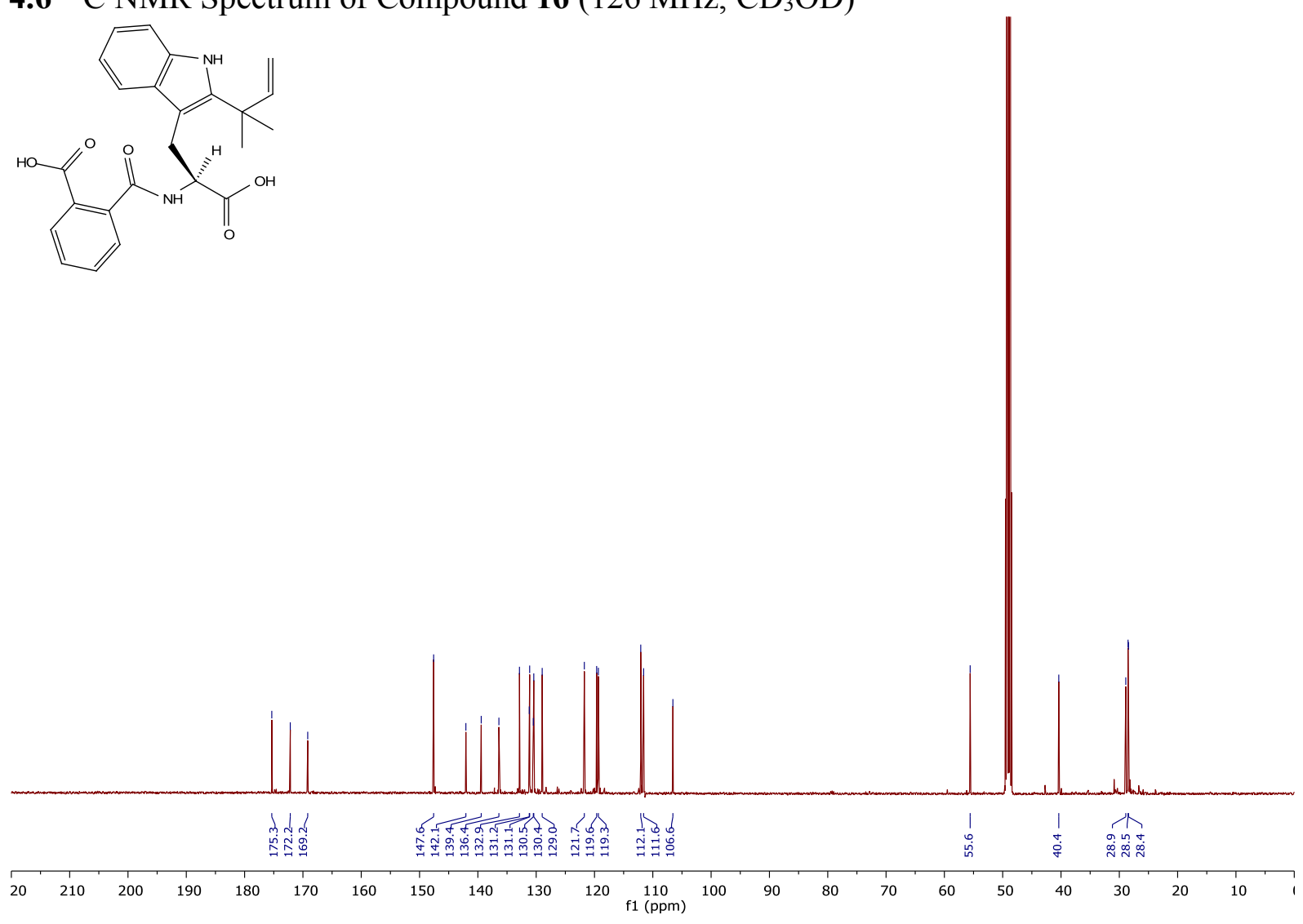


4. ${ }^{1} \mathrm{H}$ NMR Spectrum of Compound 17 (500 MHz, $\left.\mathrm{CD}_{3} \mathrm{OD}\right)$

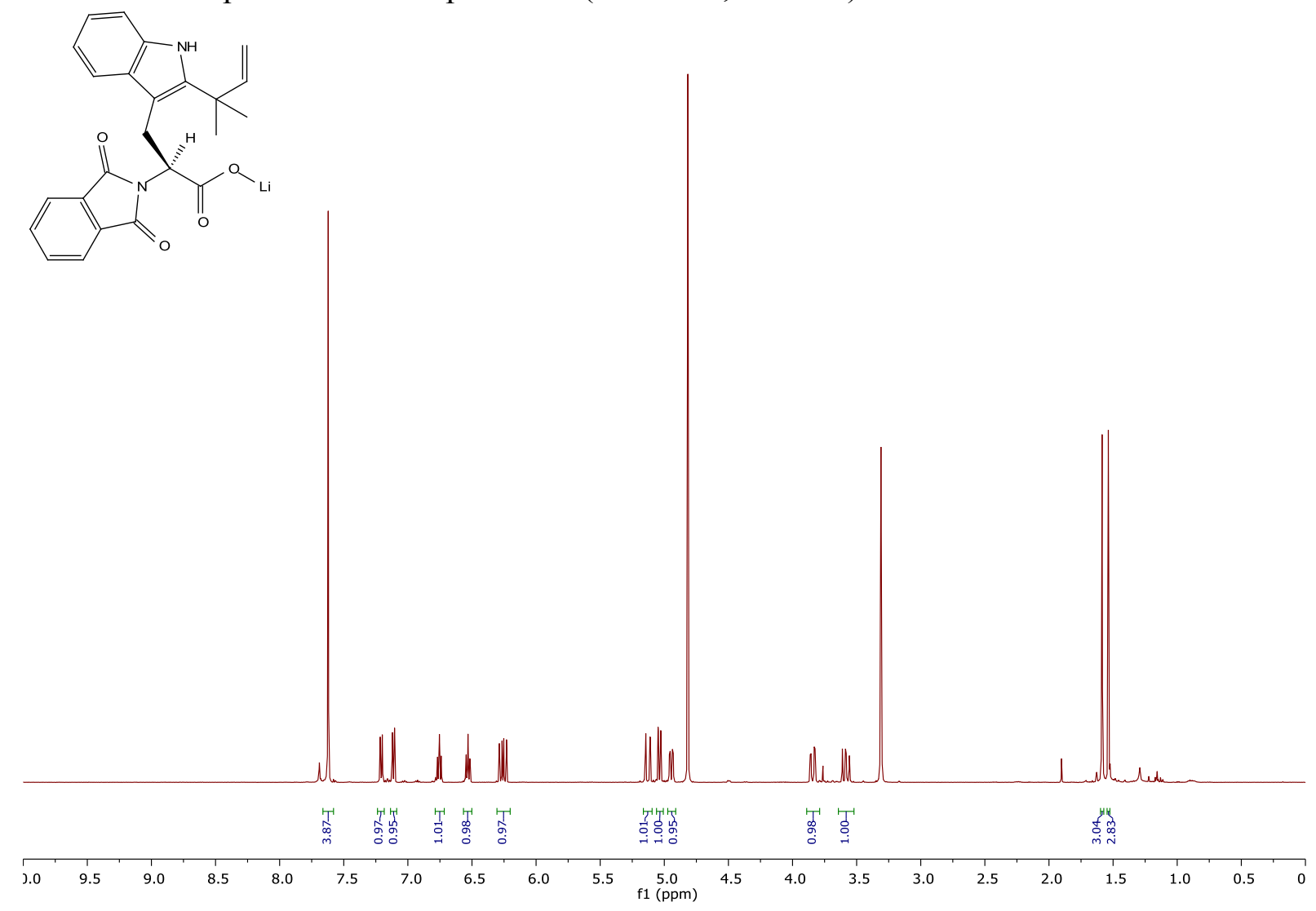

4.8 ${ }^{13} \mathrm{C}$ NMR Spectrum of Compound $17\left(126 \mathrm{MHz}, \mathrm{CD}_{3} \mathrm{OD}\right)$
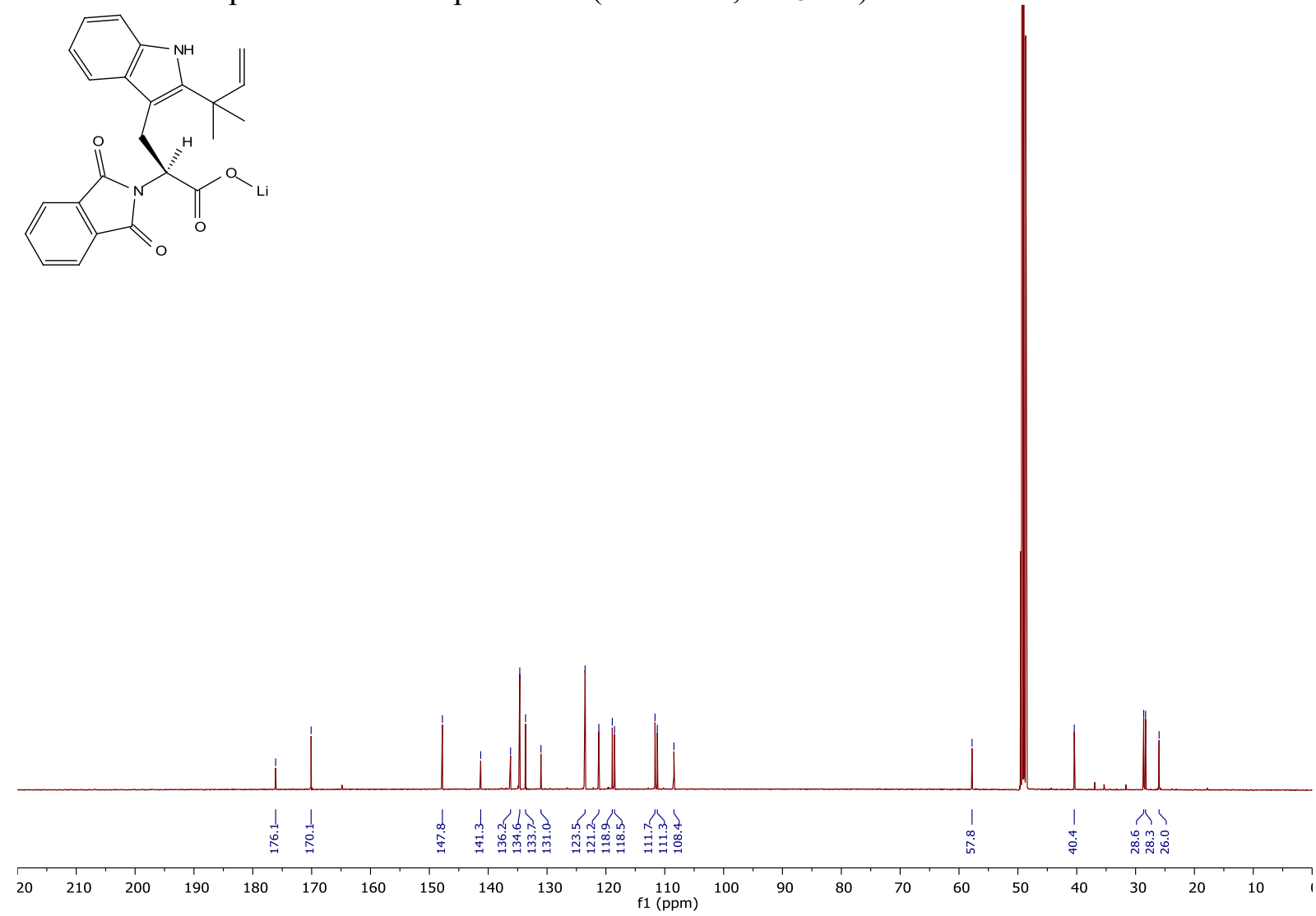
4.9 ${ }^{1} \mathrm{H}$ NMR Spectrum of Compound $19\left(500 \mathrm{MHz}, \mathrm{CD}_{2} \mathrm{Cl}_{2}\right)$

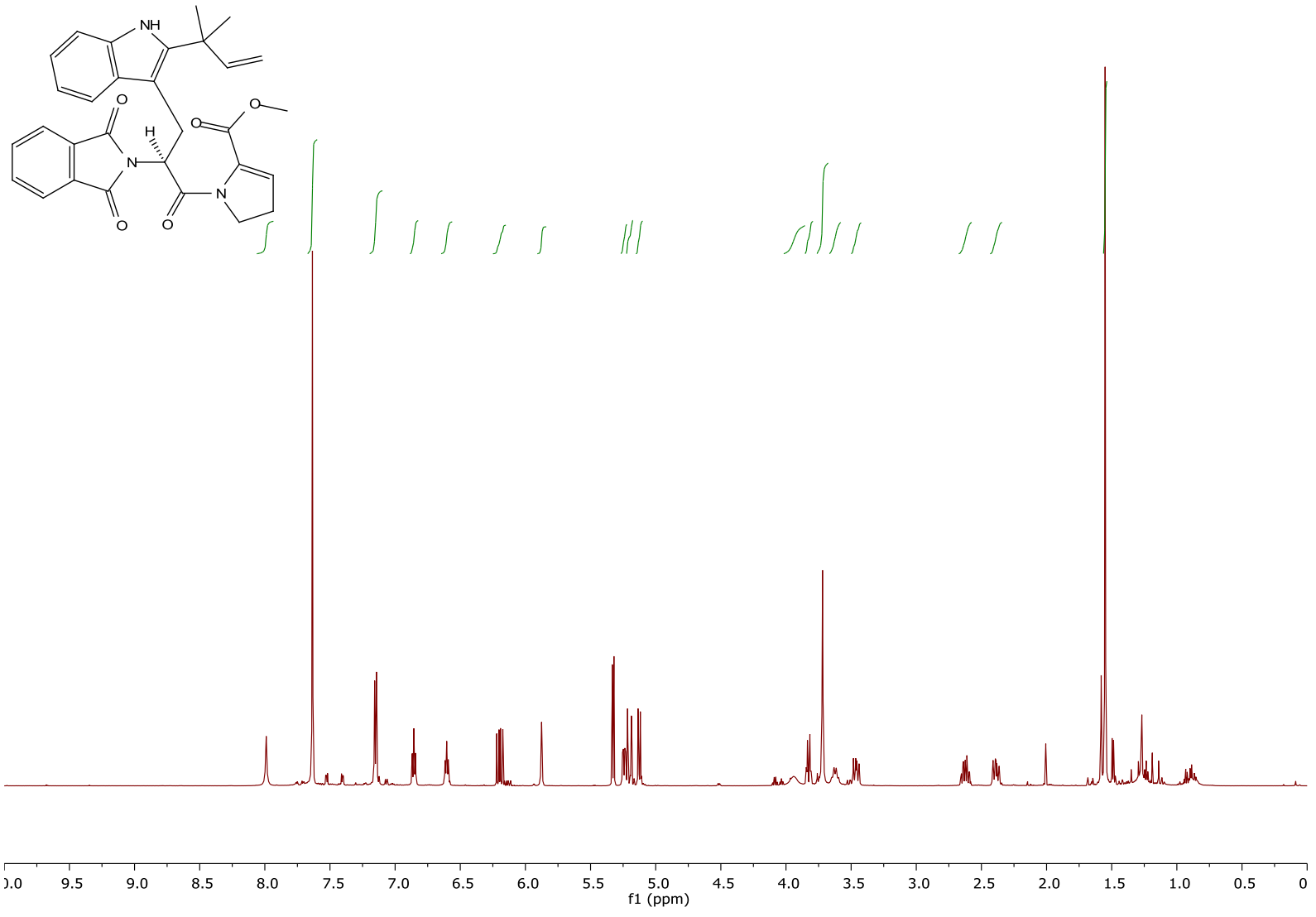

4.10 ${ }^{13} \mathrm{C}$ NMR Spectrum of Compound $19\left(151 \mathrm{MHz}, \mathrm{CD}_{2} \mathrm{Cl}_{2}\right)$
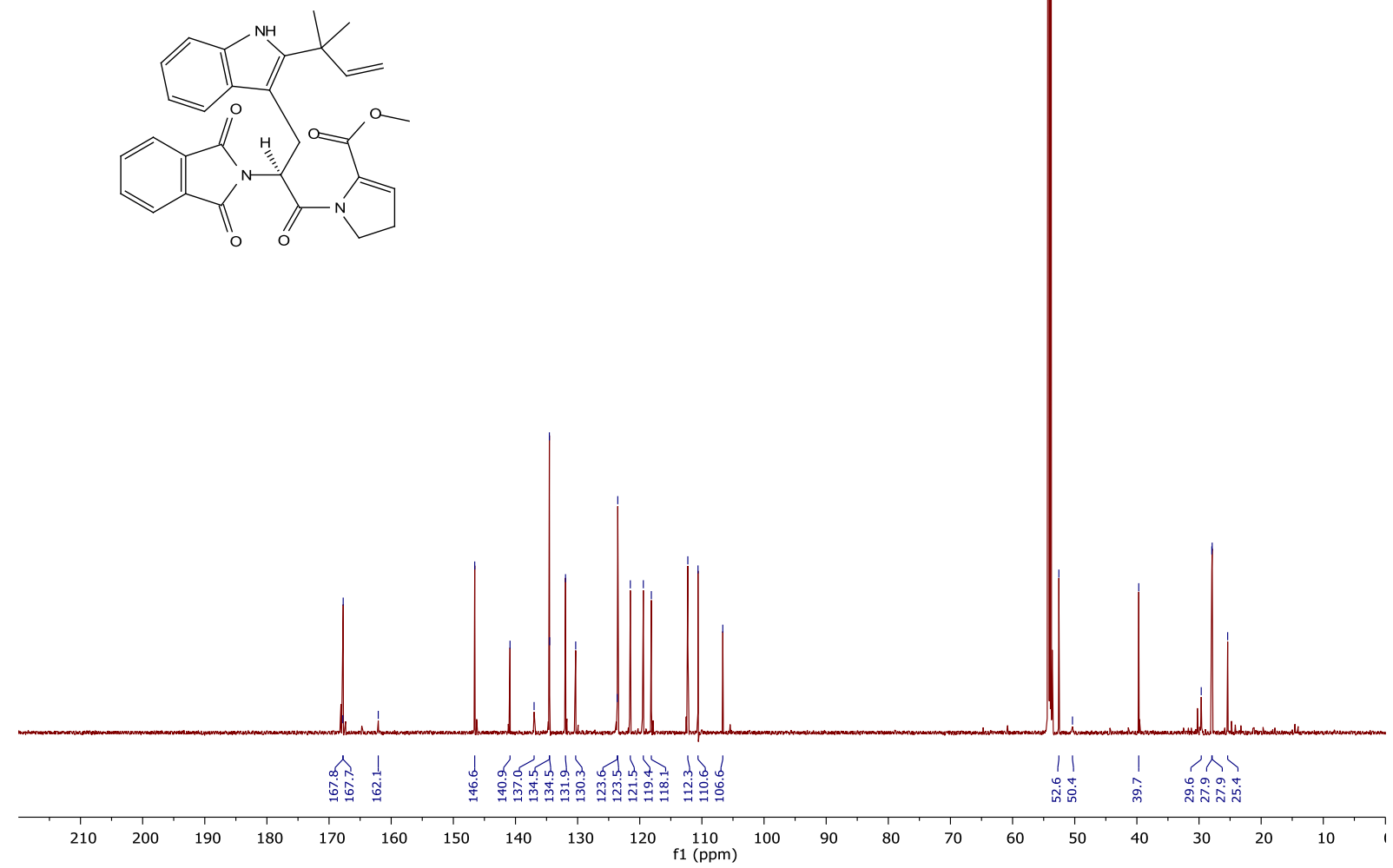
4.11 ${ }^{1} \mathrm{H}$ NMR Spectrum of Compound $9\left(500 \mathrm{MHz}, \mathrm{CDCl}_{3}\right)$

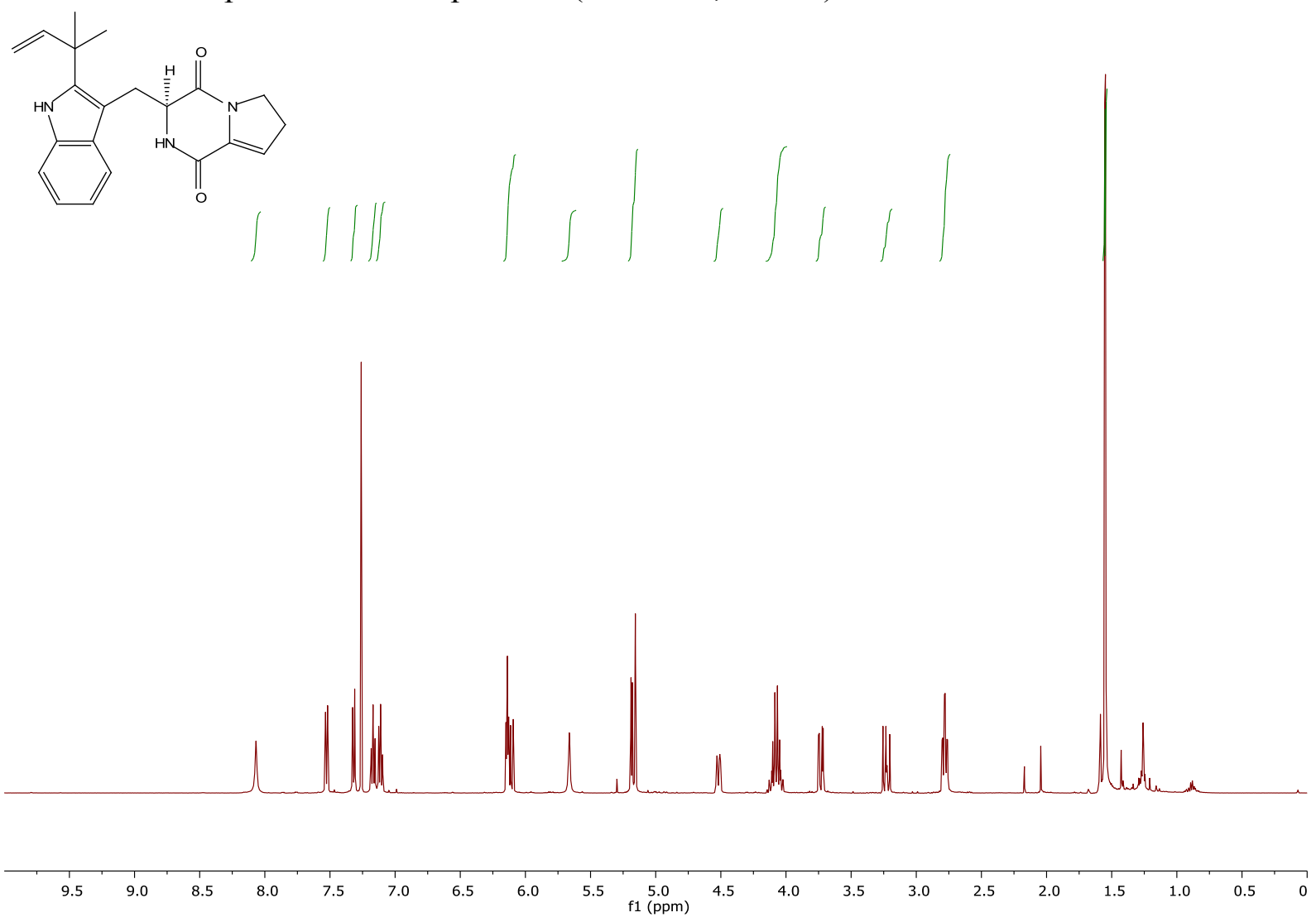

4.12 ${ }^{13} \mathrm{C}$ NMR Spectrum of Compound $9\left(126 \mathrm{MHz}, \mathrm{CDCl}_{3}\right)$<smiles>C=CC(C)(C)c1[nH]c2ccccc2c1C[C@H]1NC(=O)C2=CCCN2C1=O</smiles>

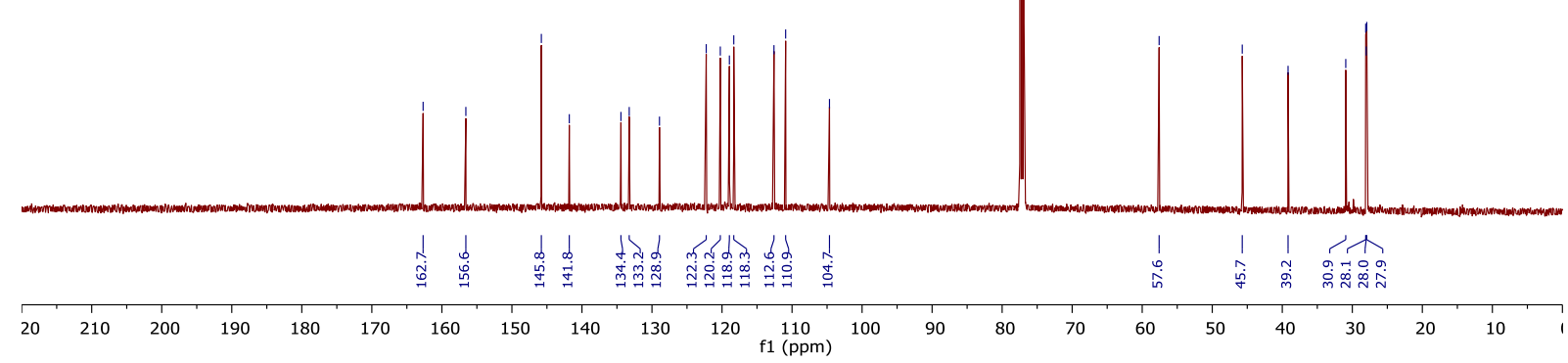


4.13 ${ }^{1} \mathrm{H}$ NMR Spectrum of Compound $12\left(500 \mathrm{MHz}, \mathrm{CDCl}_{3}\right)$<smiles>C=CC(C)(C)[C@]12Nc3ccccc3[C@@]1(C)C[C@]1(C)C(=O)N3CCC=C3C(=O)N12</smiles>
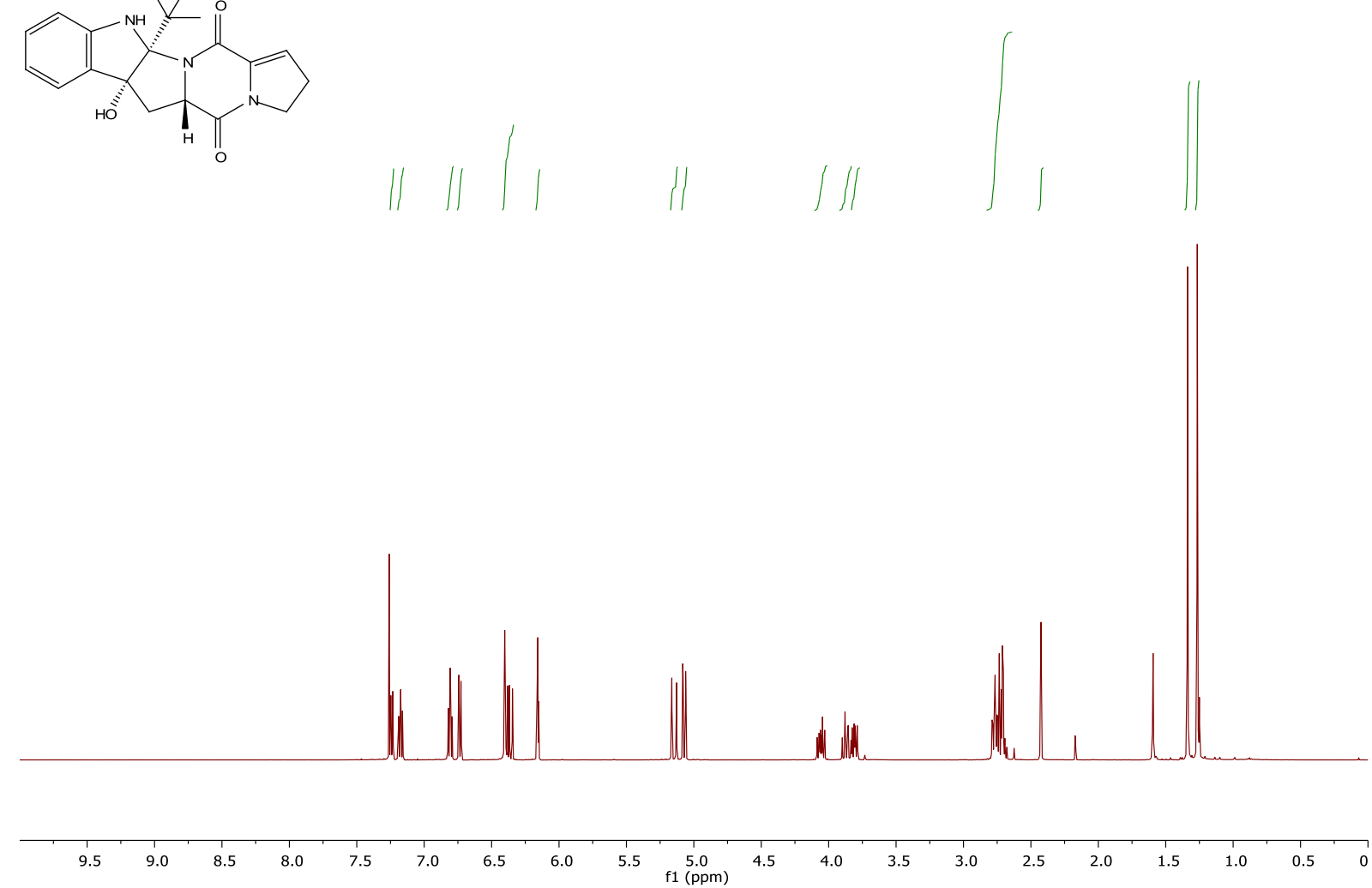

4.14 ${ }^{13} \mathrm{C}$ NMR Spectrum of Compound $12\left(126 \mathrm{MHz}, \mathrm{CDCl}_{3}\right)$<smiles>C=CC(C)(C)[C@]12Nc3ccccc3[C@@]1(C)C[C@]1(O)C(=O)N3CCC=C3C(=O)N12</smiles>

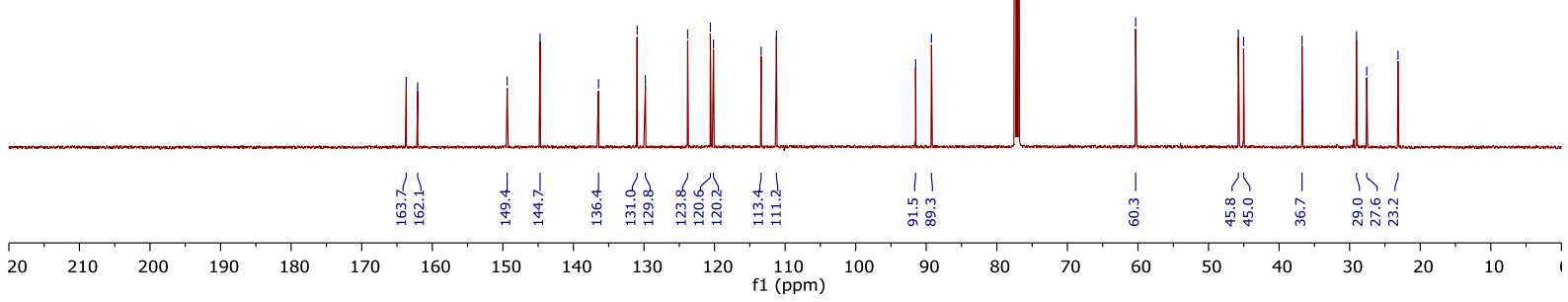


4.15 ${ }^{1} \mathrm{H}$ NMR Spectrum of Compound dia-12 (500 MHz, $\left.\mathrm{CDCl}_{3}\right)$

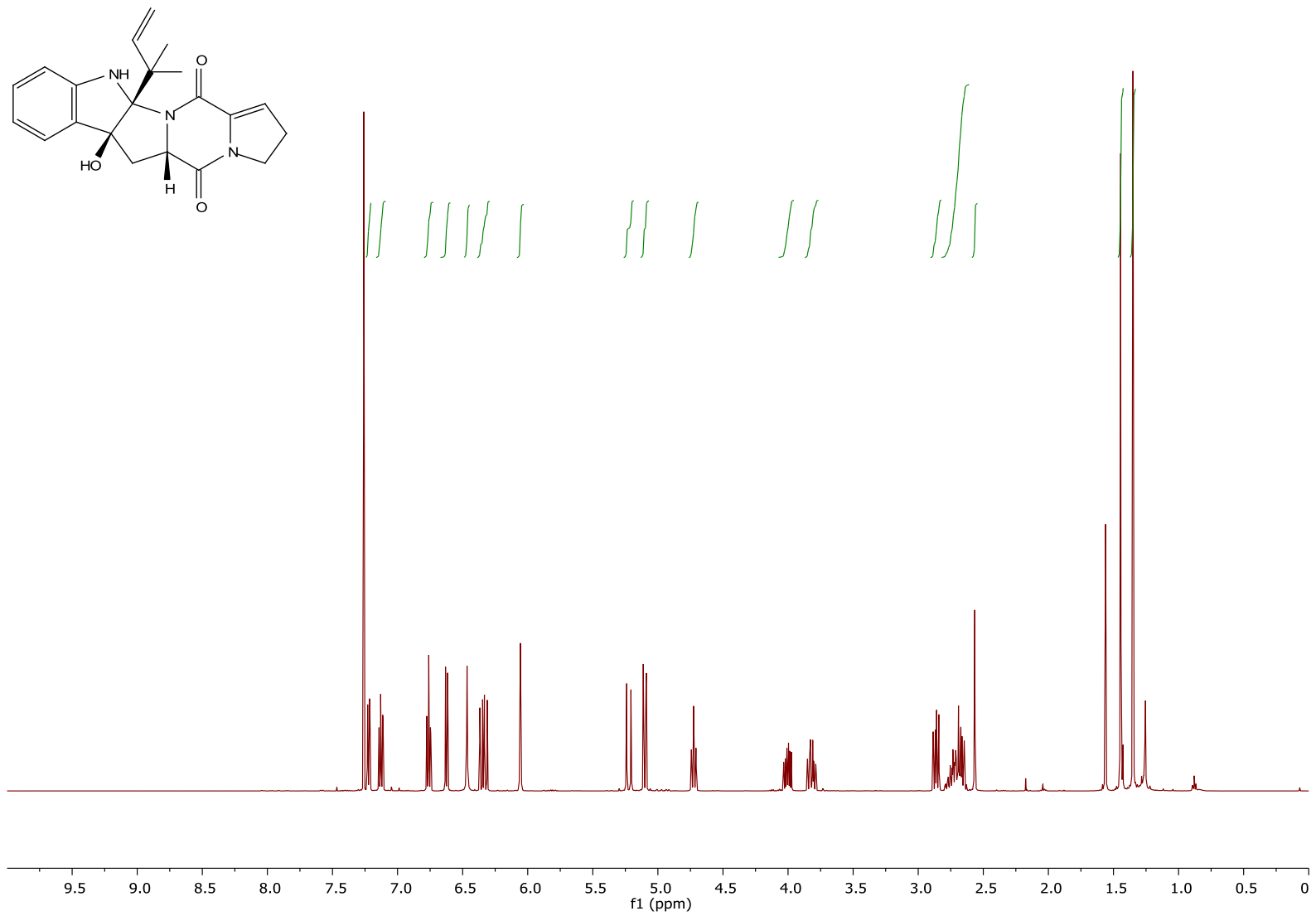

4.16 ${ }^{13} \mathrm{C}$ NMR Spectrum of Compound dia-12 (126 MHz, $\left.\mathrm{CDCl}_{3}\right)$

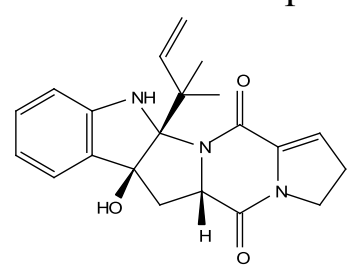

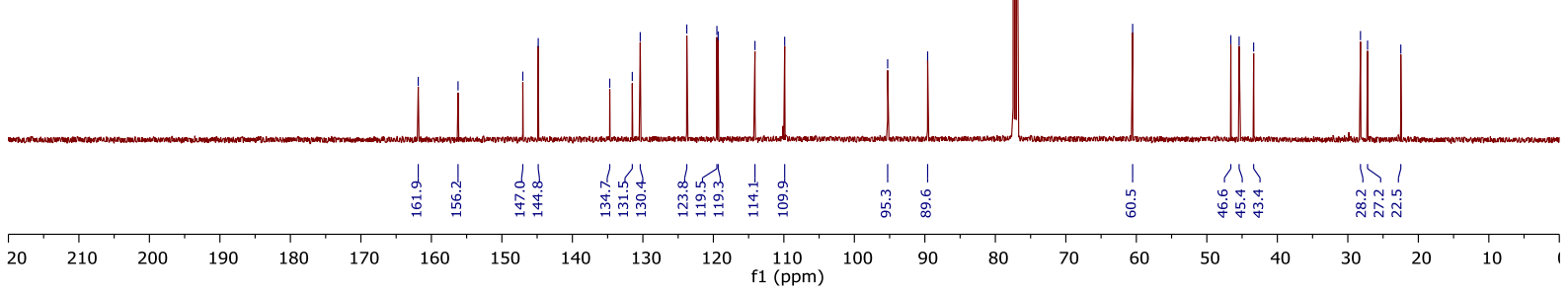


4.17 ${ }^{1} \mathrm{H}$ NMR Spectrum of Brevianamide A (1) $\left(500 \mathrm{MHz}, \mathrm{CDCl}_{3}, \sim 3.0 \mathrm{mg} / \mathrm{mL}\right)$
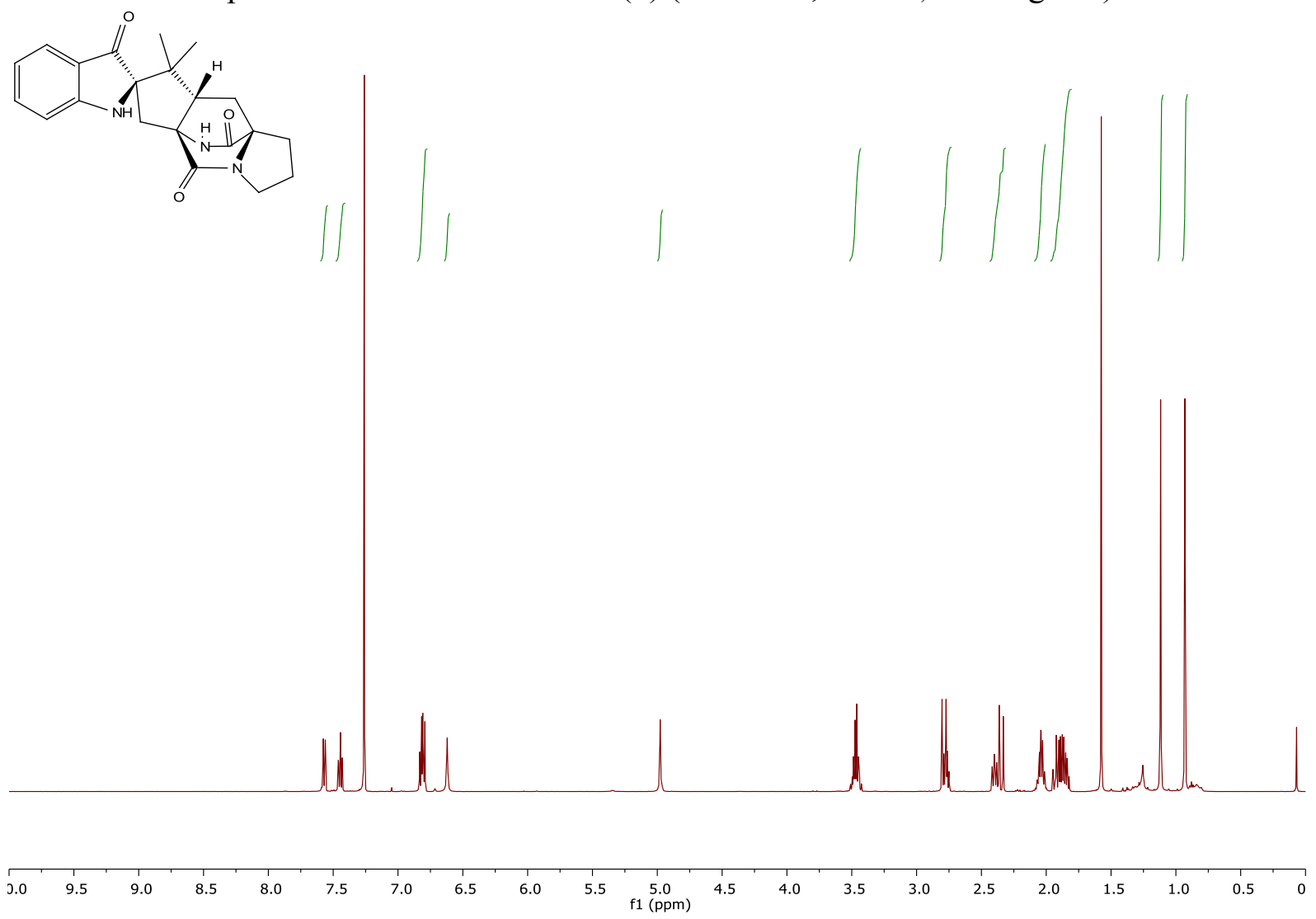

4.18 ${ }^{13} \mathrm{C}$ NMR Spectrum of Brevianamide A (1) (126 MHz, $\mathrm{CDCl}_{3}, \sim 3.0 \mathrm{mg} / \mathrm{mL}$ )
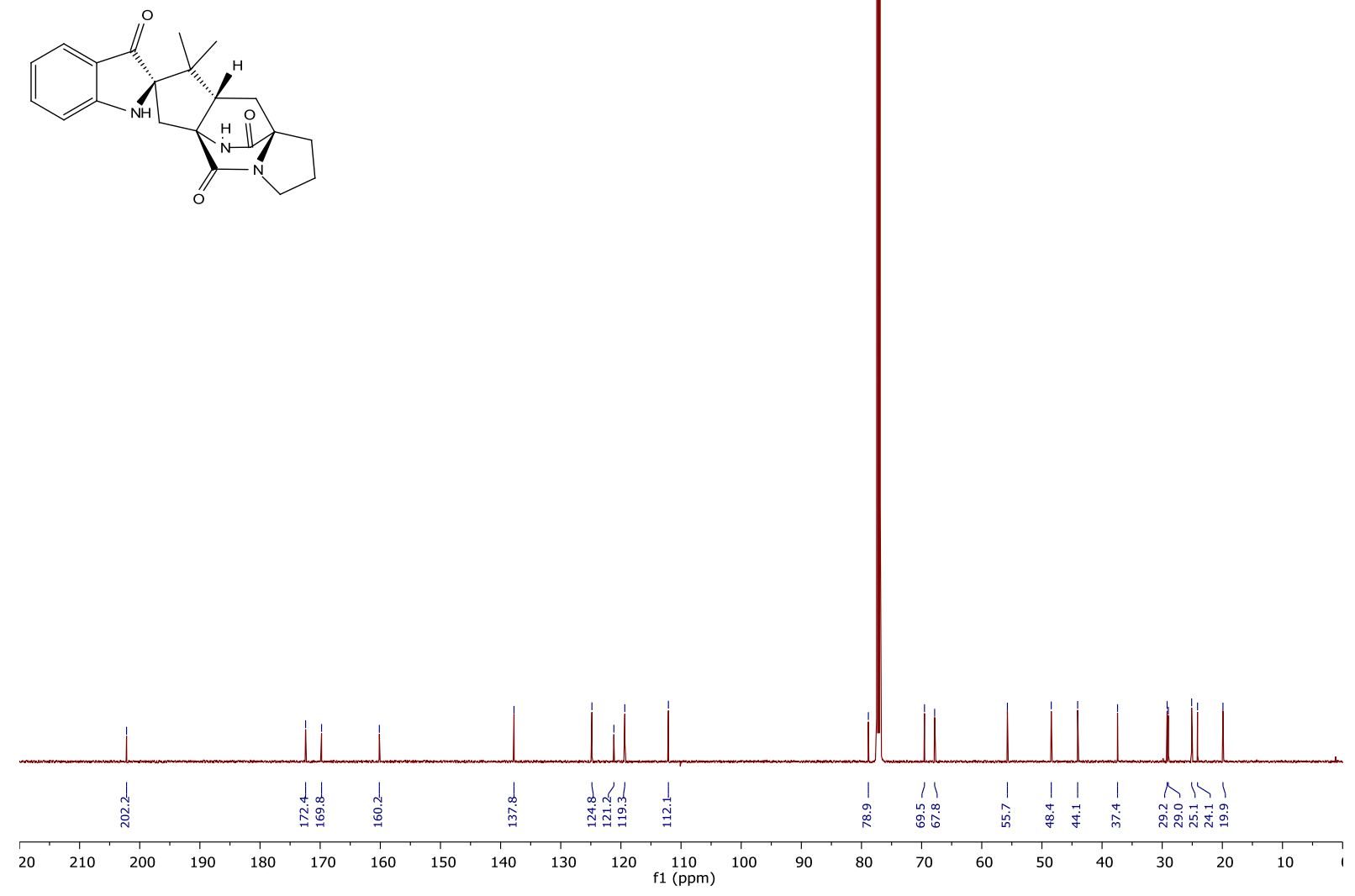

39 
4.19 ${ }^{1} \mathrm{H}-{ }^{1} \mathrm{H}$ COSY Spectrum of Brevianamide A (1) $\left(\mathrm{CDCl}_{3}\right)$

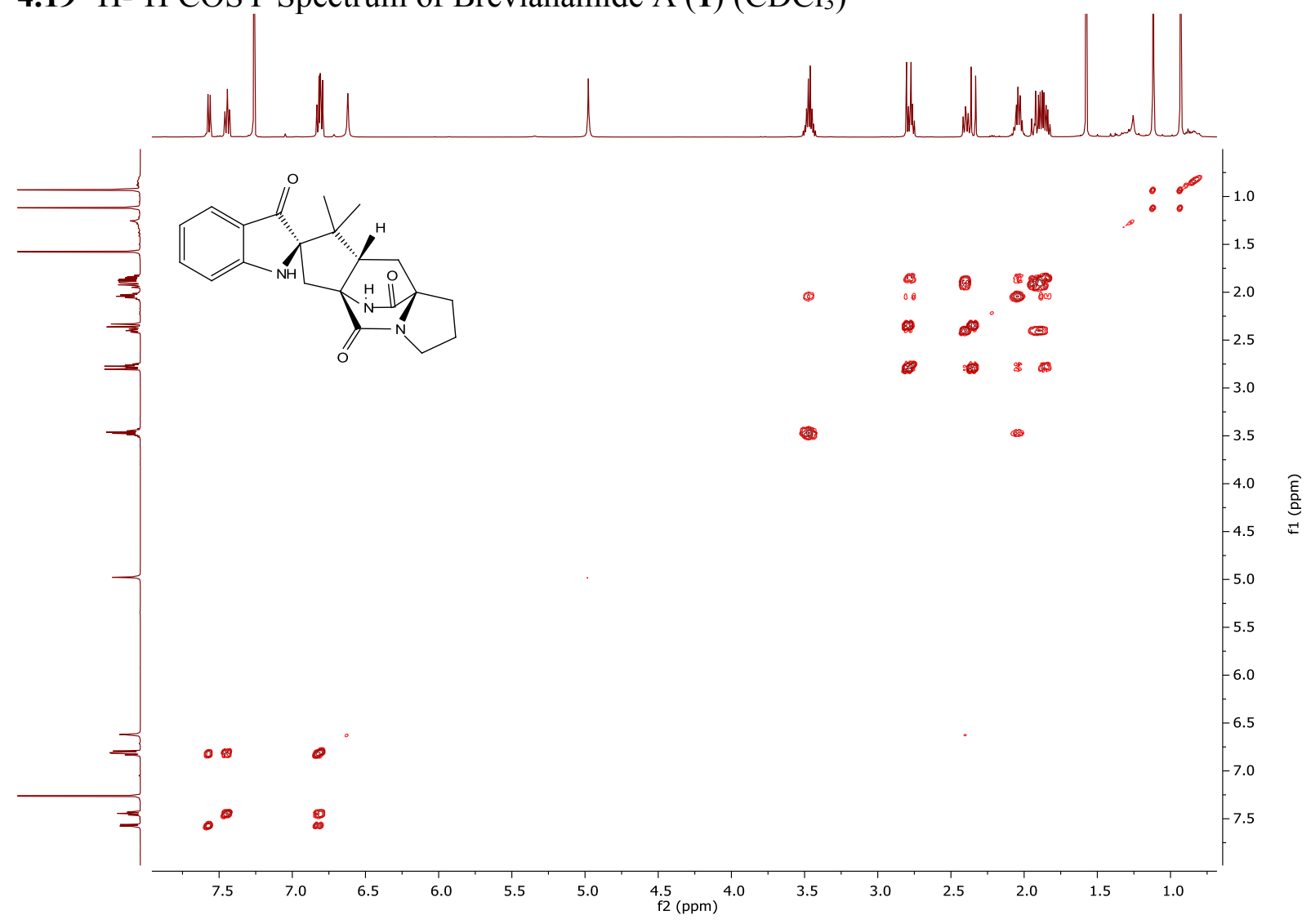

4.20 ${ }^{1} \mathrm{H}_{-}{ }^{13} \mathrm{C}$ HSQC Spectrum of Brevianamide A (1) $\left(\mathrm{CDCl}_{3}\right)$

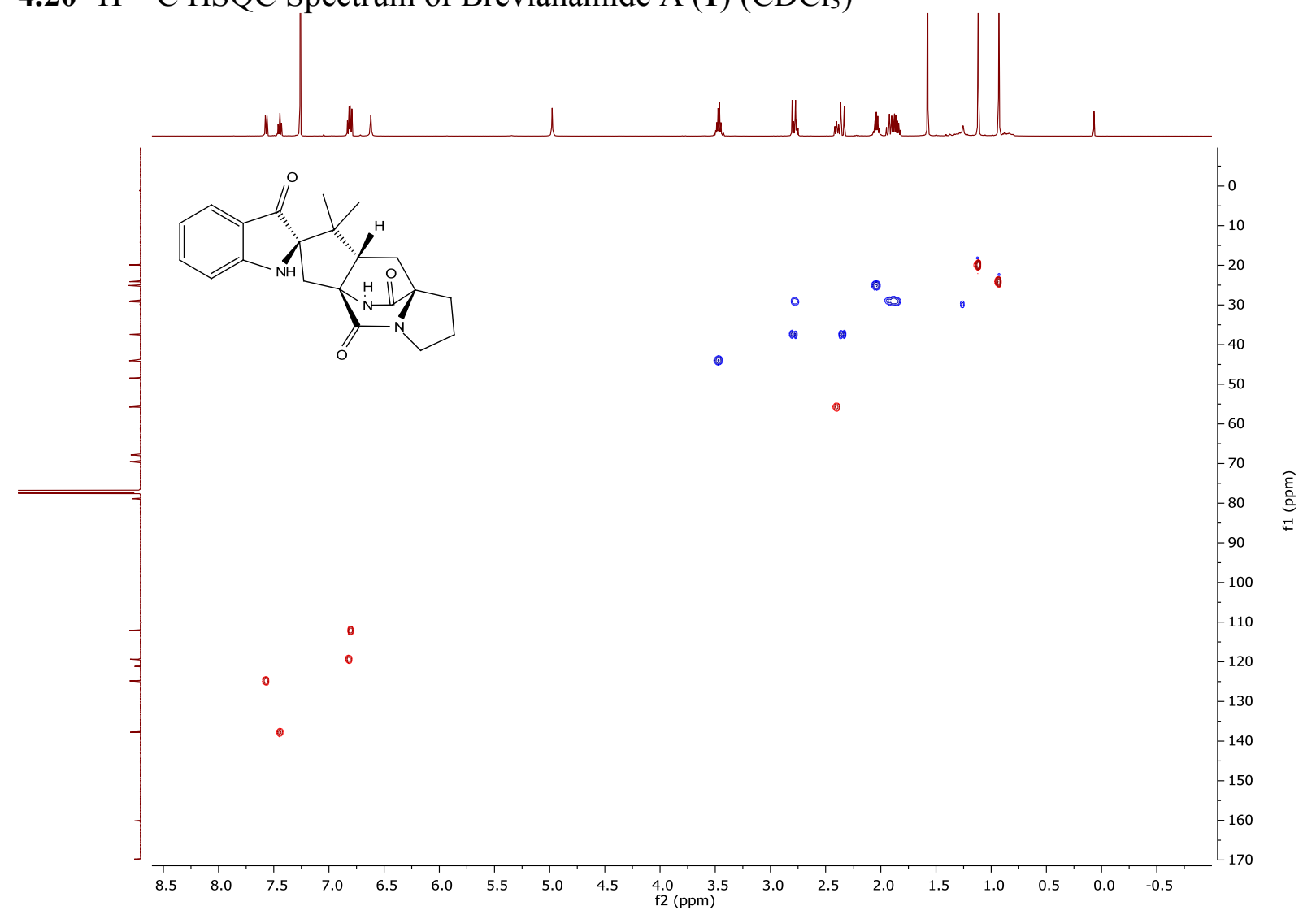


4.21 ${ }^{1} \mathrm{H}-{ }^{13} \mathrm{C}$ HMBC Spectrum of Brevianamide A (1) $\left(\mathrm{CDCl}_{3}\right)$

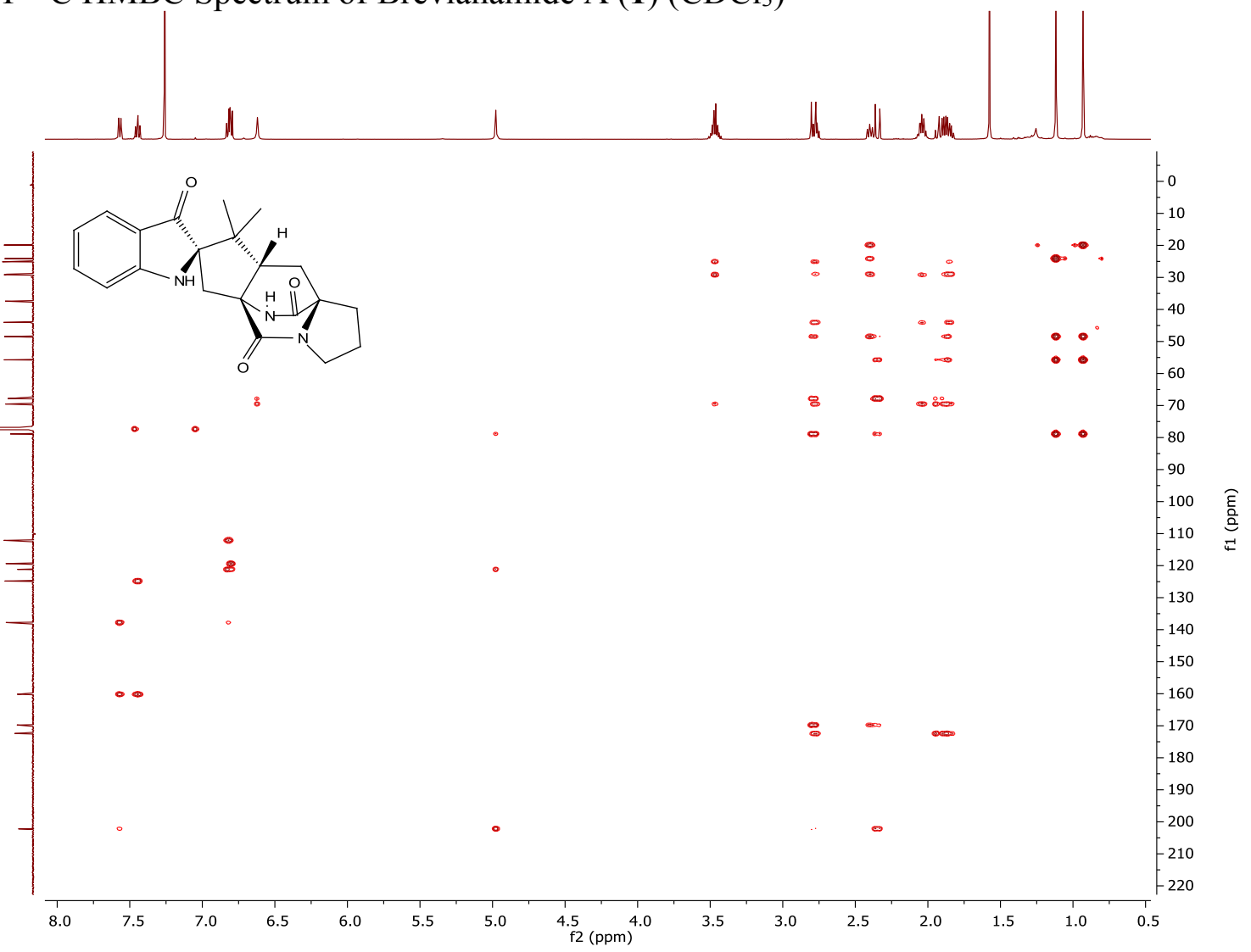

4.22 ${ }^{1} \mathrm{H}-{ }^{1} \mathrm{H}$ NOESY Spectrum of Brevianamide A (1) $\left(\mathrm{CDCl}_{3}\right)$

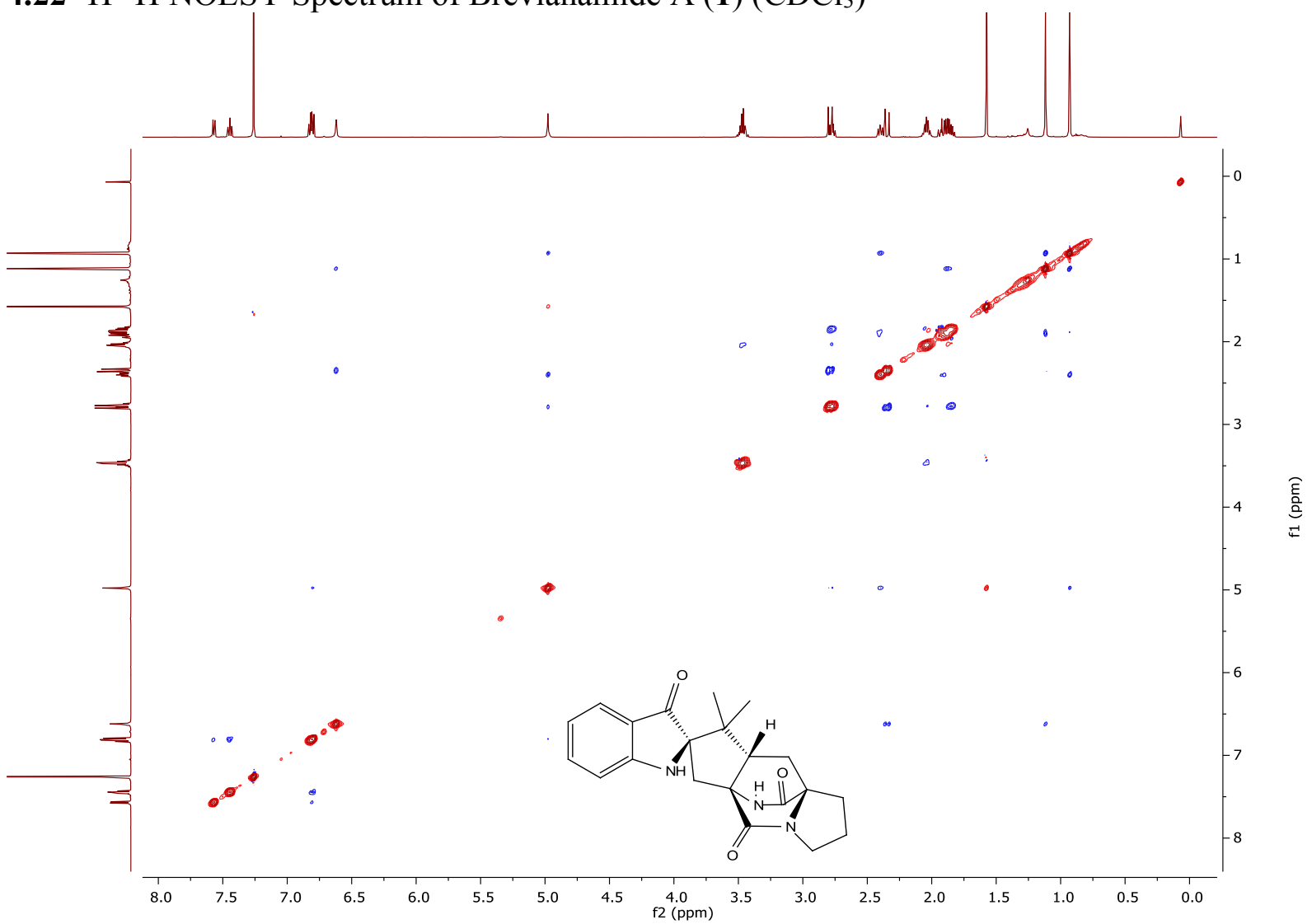

41 

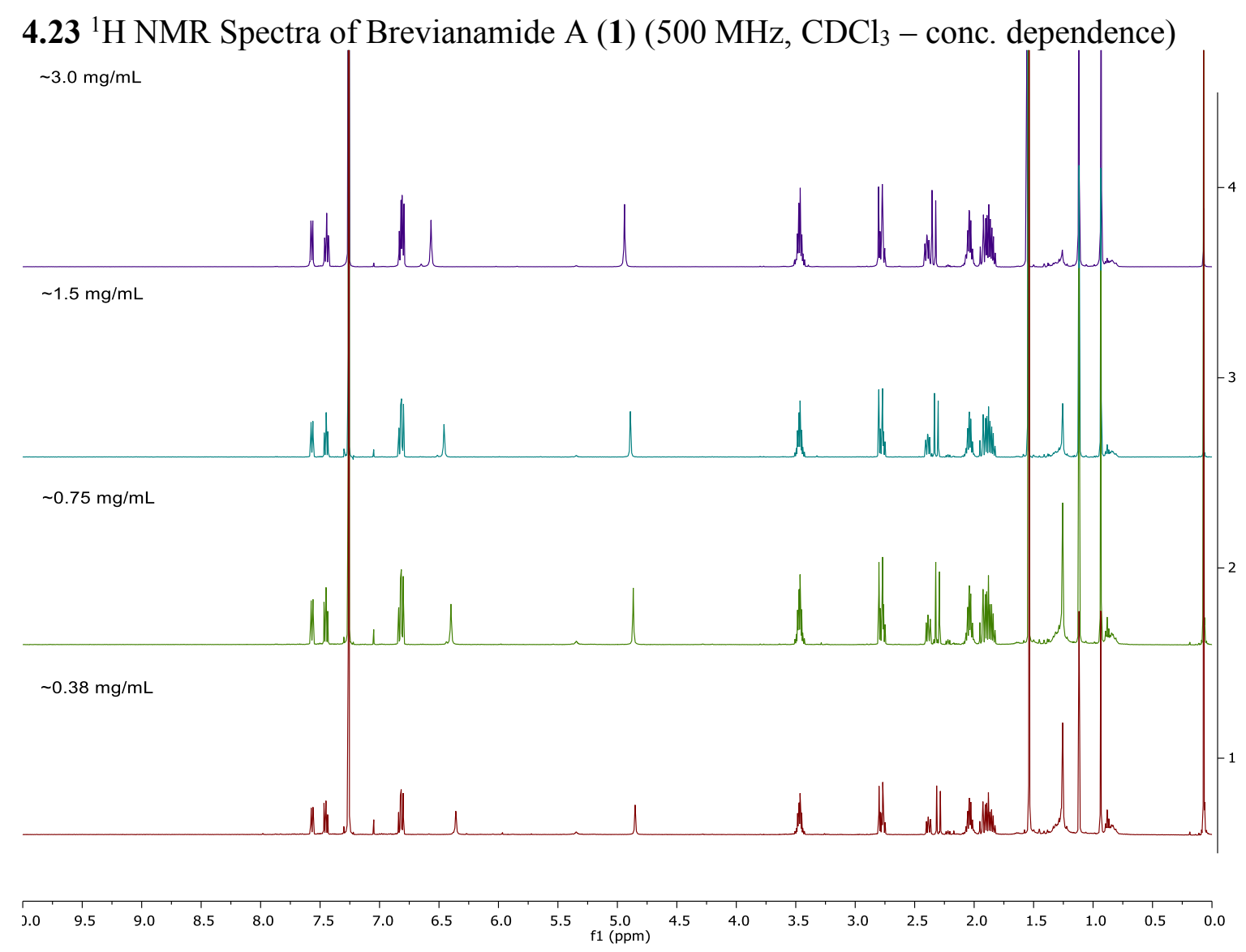

$\sim 3.0 \mathrm{mg} / \mathrm{mL}$
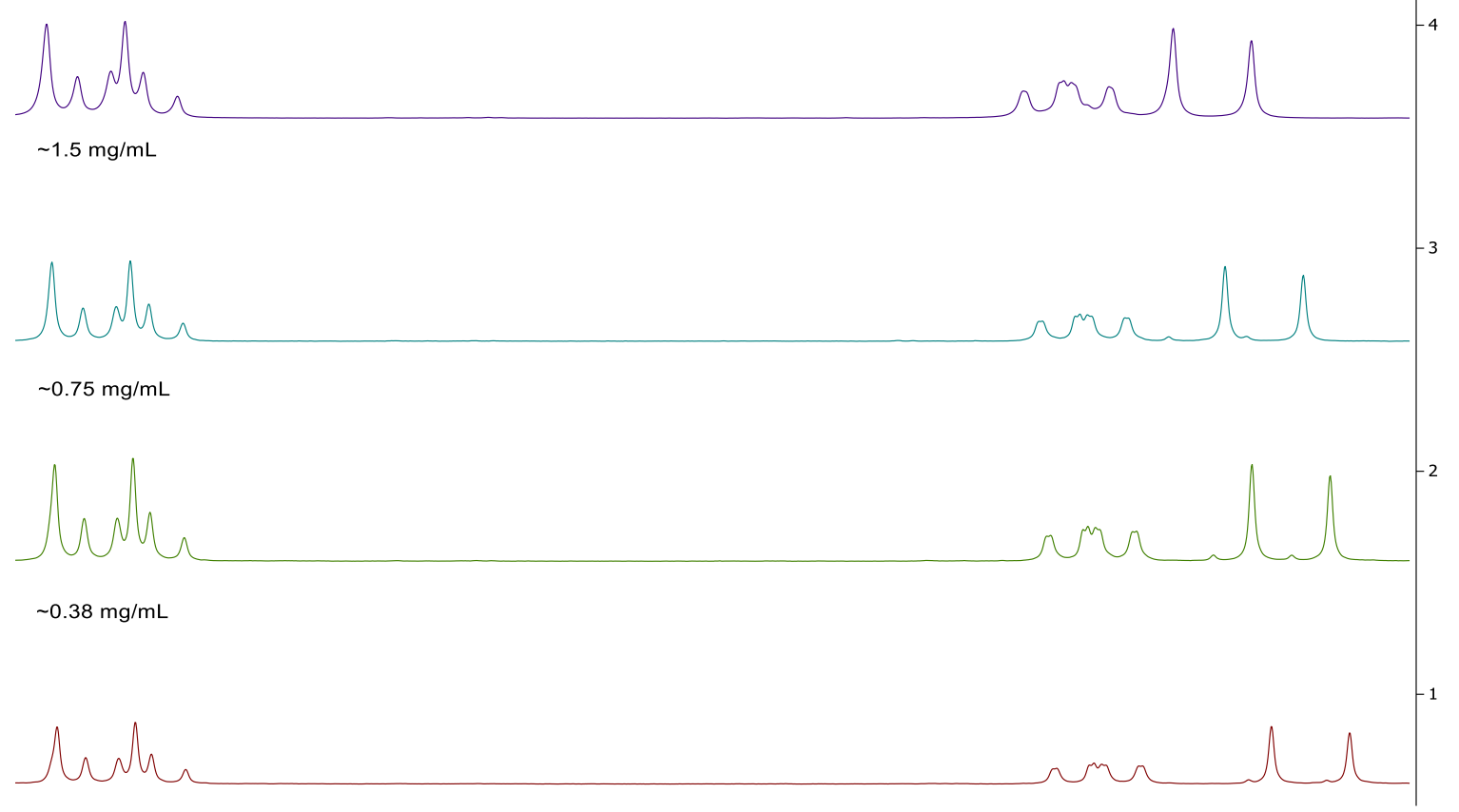

$\begin{array}{llllllllllllllllllllllllllllllllll}2.80 & 2.78 & 2.76 & 2.74 & 2.72 & 2.70 & 2.68 & 2.66 & 2.64 & 2.62 & 2.60 & 2.58 & 2.56 & 2.54 & 2.52 & 2.50 & 2.48 & 2.46 & 2.44 & 2.42 & 2.40 & 2.38 & 2.36 & 2.34 & 2.32 & 2.30 & 2.28 & 2.26\end{array}$

42 
4.24 ${ }^{1} \mathrm{H}$ NMR Spectrum of Brevianamide A (1) $\left(500 \mathrm{MHz},\left(\mathrm{CD}_{3}\right)_{2} \mathrm{SO}\right)$
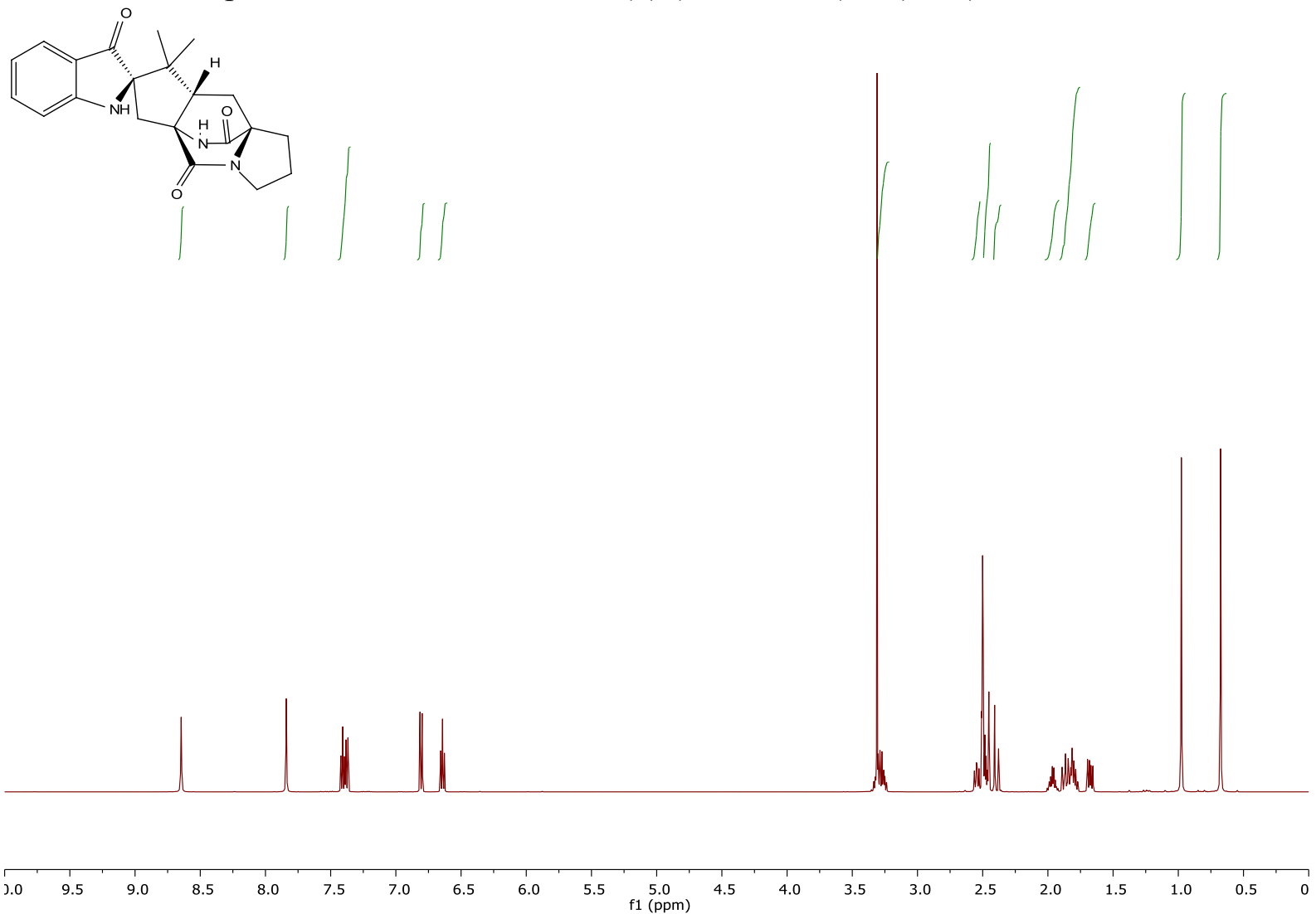

4.25 ${ }^{13} \mathrm{C}$ NMR Spectrum of Brevianamide A (1) (126 MHz, $\left.\left(\mathrm{CD}_{3}\right)_{2} \mathrm{SO}\right)$
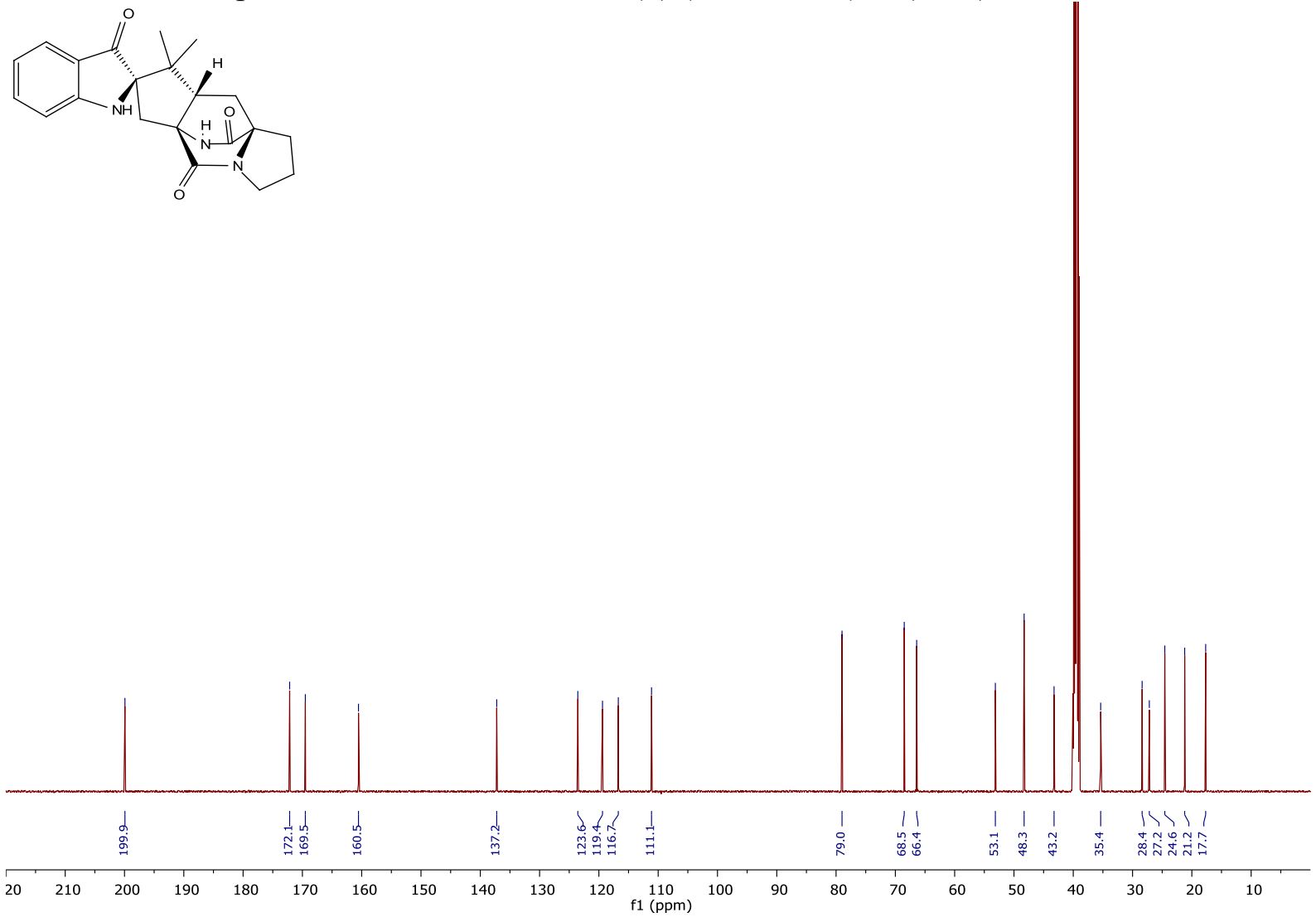
4.26 ${ }^{1} \mathrm{H}-{ }^{1} \mathrm{H}$ COSY Spectrum of Brevianamide A (1) $\left(\left(\mathrm{CD}_{3}\right)_{2} \mathrm{SO}\right)$

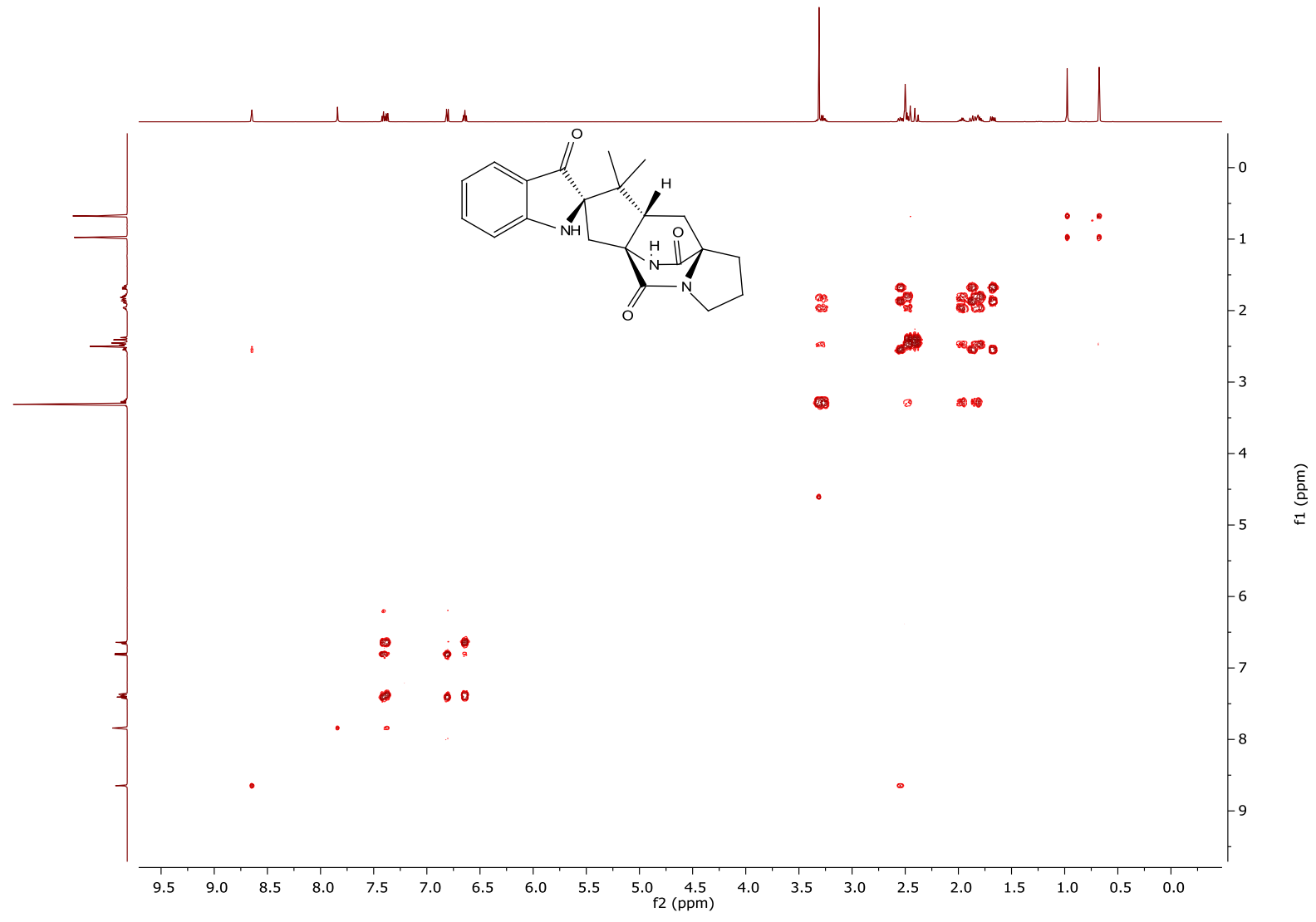

4.27 ${ }^{1} \mathrm{H}-{ }^{13} \mathrm{C}$ HSQC Spectrum of Brevianamide A (1) $\left(\left(\mathrm{CD}_{3}\right)_{2} \mathrm{SO}\right)$

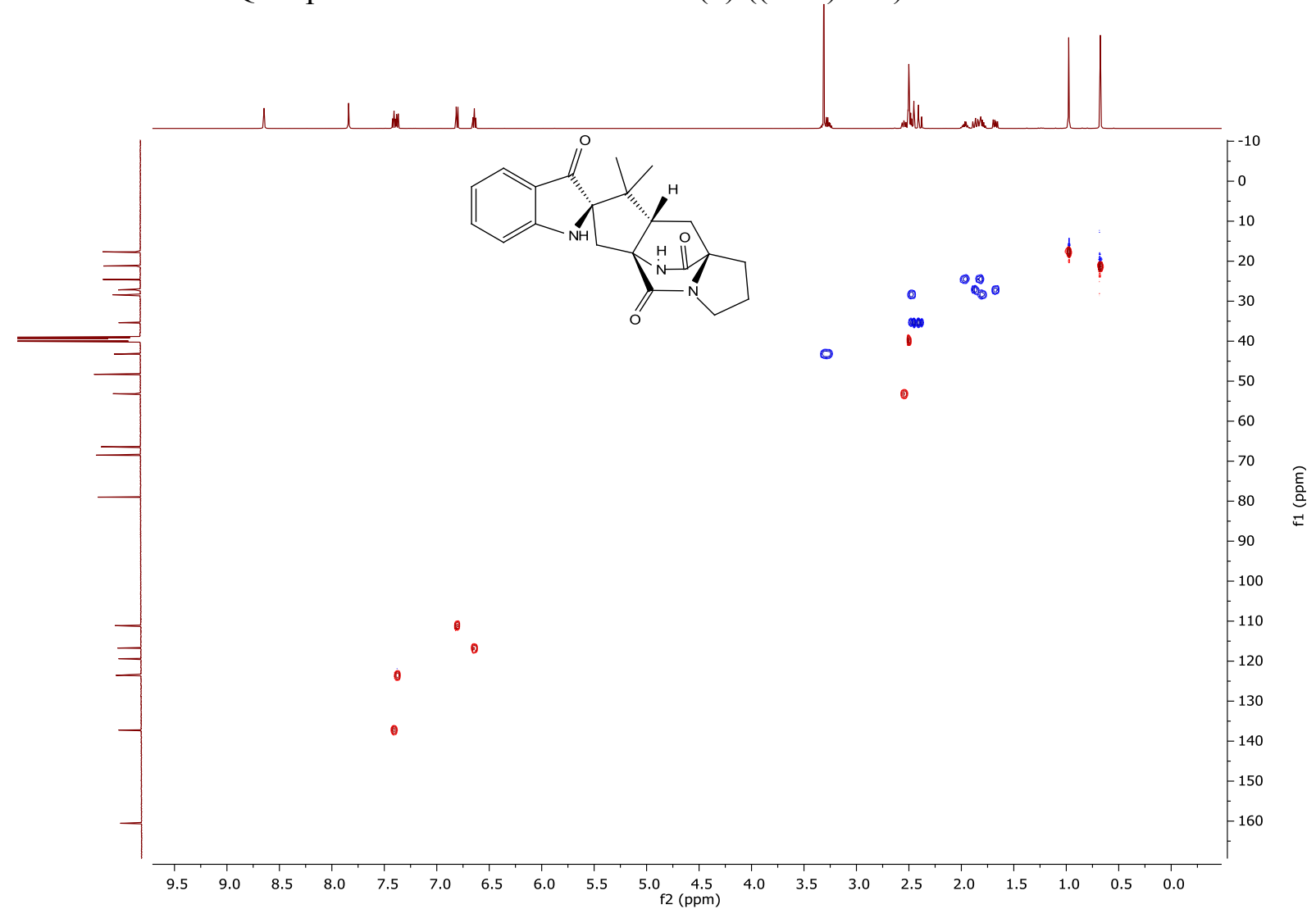


4.28 ${ }^{1} \mathrm{H}-{ }^{13} \mathrm{C}$ HMBC Spectrum of Brevianamide A (1) $\left(\left(\mathrm{CD}_{3}\right)_{2} \mathrm{SO}\right)$

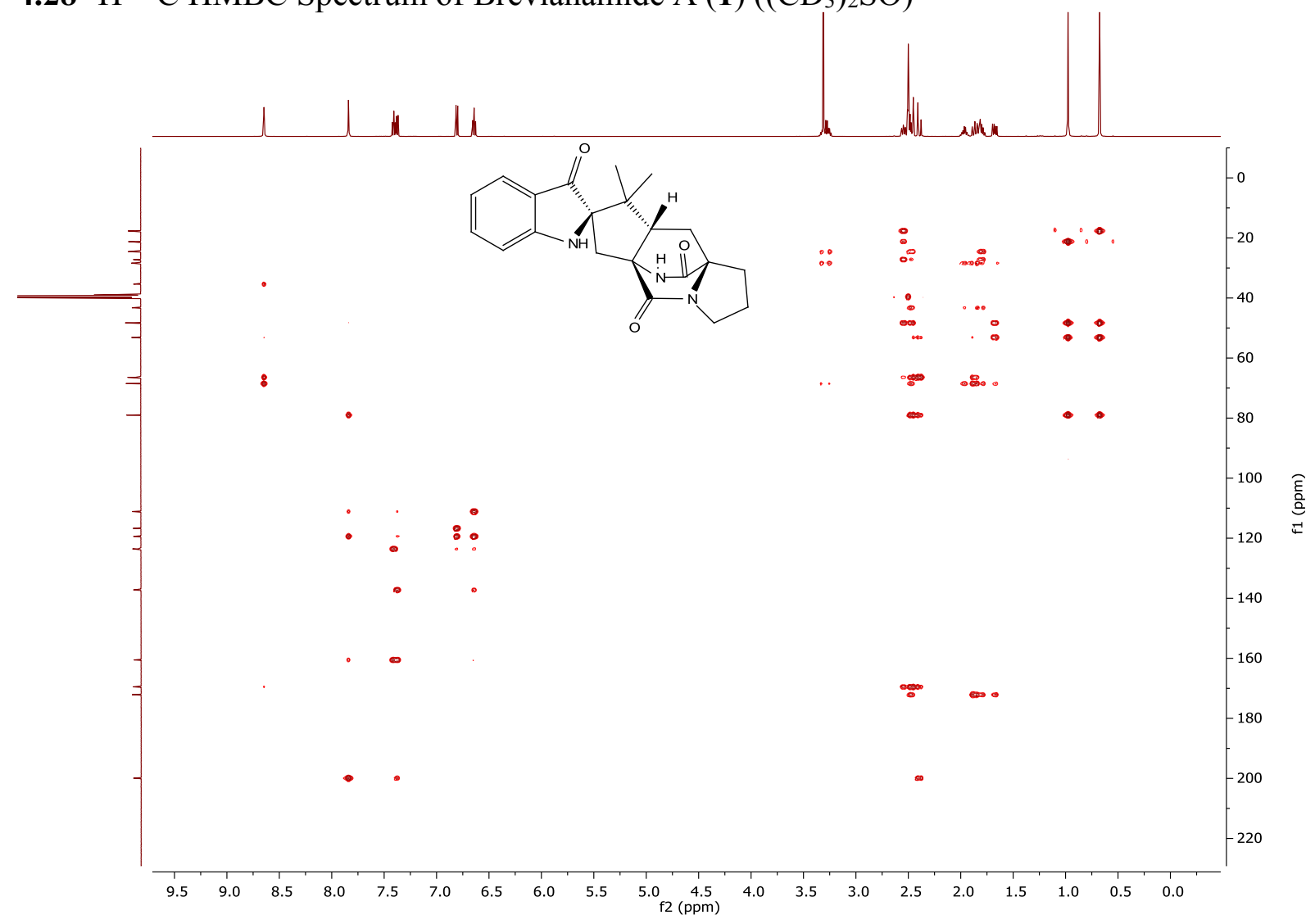

4.29 ${ }^{1} \mathrm{H}-{ }^{1} \mathrm{H}$ NOESY Spectrum of Brevianamide A (1) $\left(\left(\mathrm{CD}_{3}\right)_{2} \mathrm{SO}\right)$

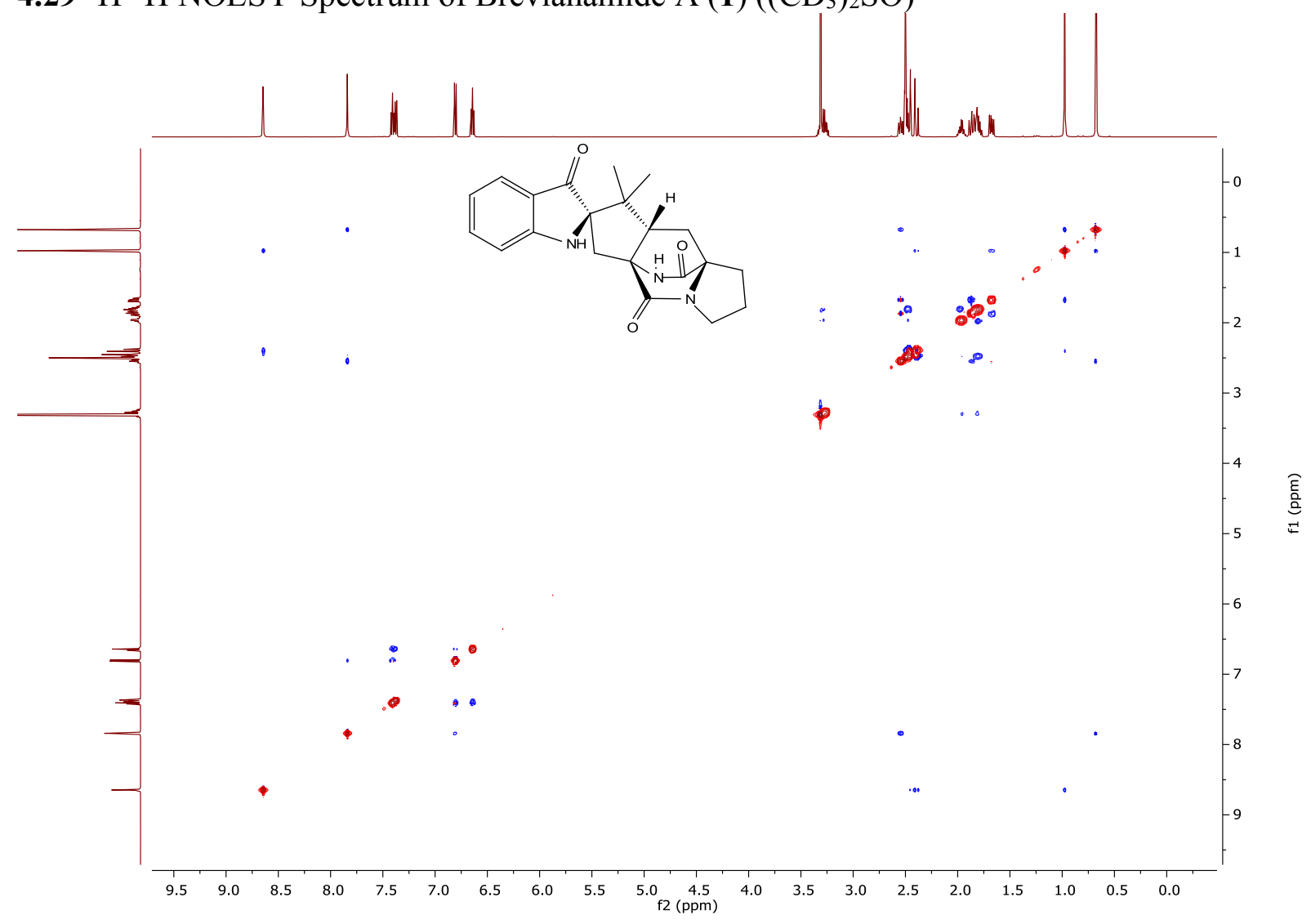


4.30 ${ }^{1} \mathrm{H}$ NMR Spectrum of Brevianamide B (2) $\left(500 \mathrm{MHz}, \mathrm{CDCl}_{3}\right)$
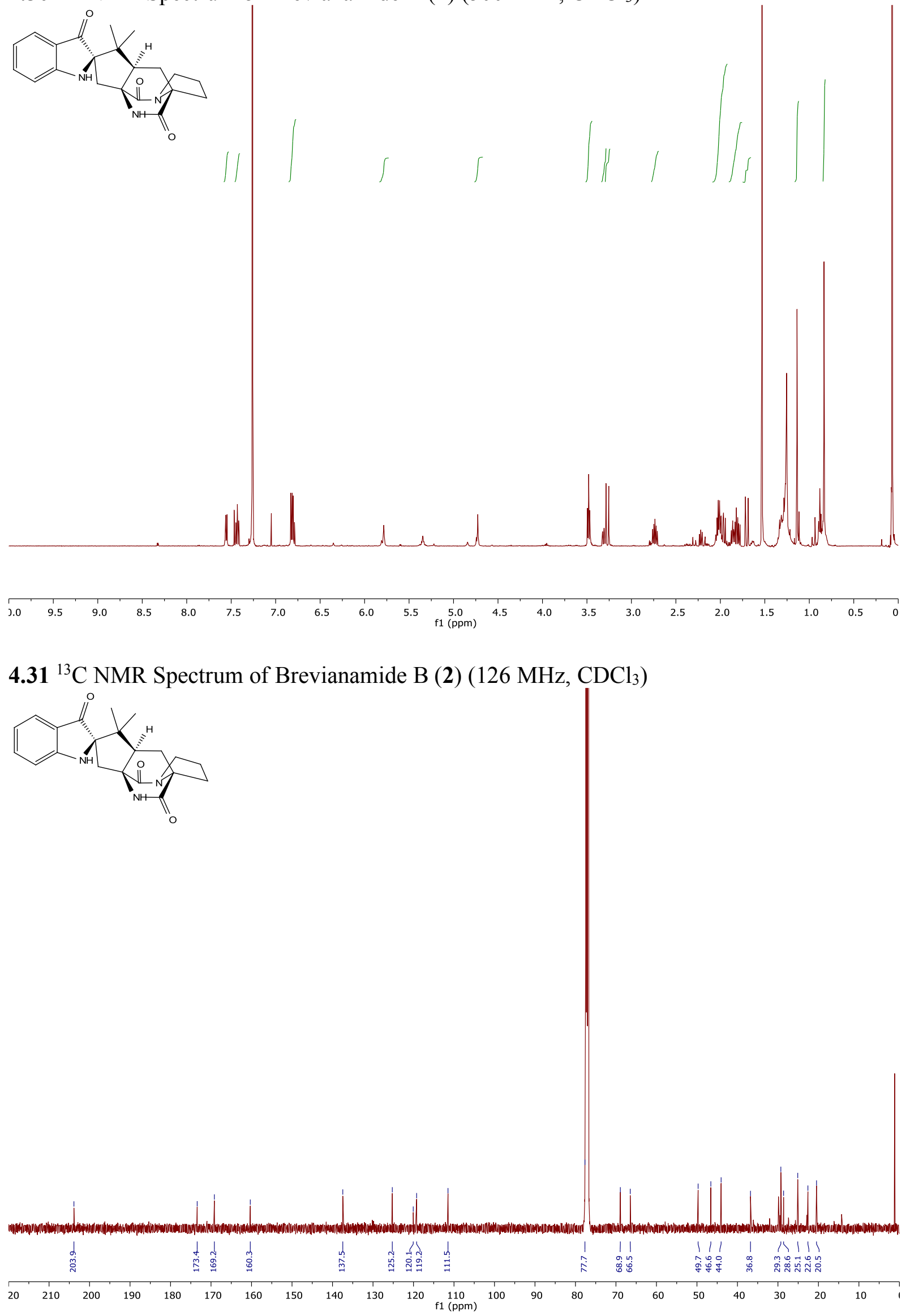

46 
4.32 ${ }^{1} \mathrm{H}$ NMR Spectrum of Brevianamide B (2) (500 MHz, $\left.\left(\mathrm{CD}_{3}\right)_{2} \mathrm{SO}\right)$
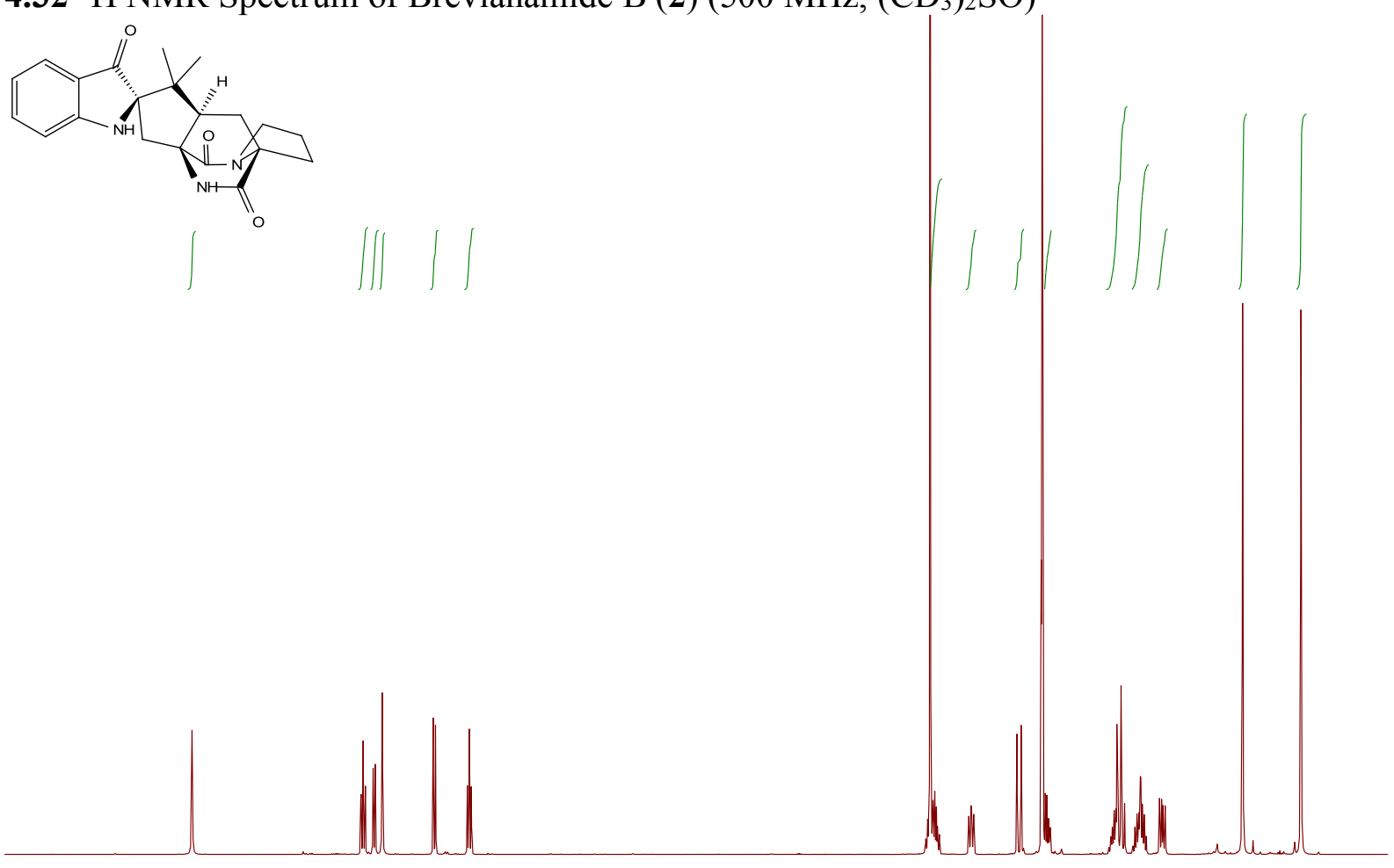

3.0

4.33 ${ }^{13} \mathrm{C}$ NMR Spectrum of Brevianamide B (2) (126 MHz, $\left.\left(\mathrm{CD}_{3}\right)_{2} \mathrm{SO}\right)$

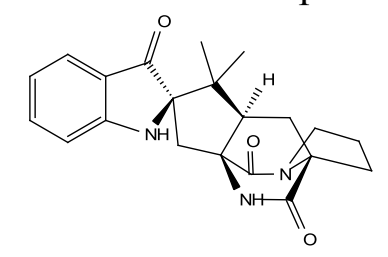

$5.5 \underset{f 1(p p m)}{5.0} 4.5$ 
4.34 ${ }^{1} \mathrm{H}-{ }^{1} \mathrm{H}$ COSY Spectrum of Brevianamide B (2) $\left(\left(\mathrm{CD}_{3}\right)_{2} \mathrm{SO}\right)$

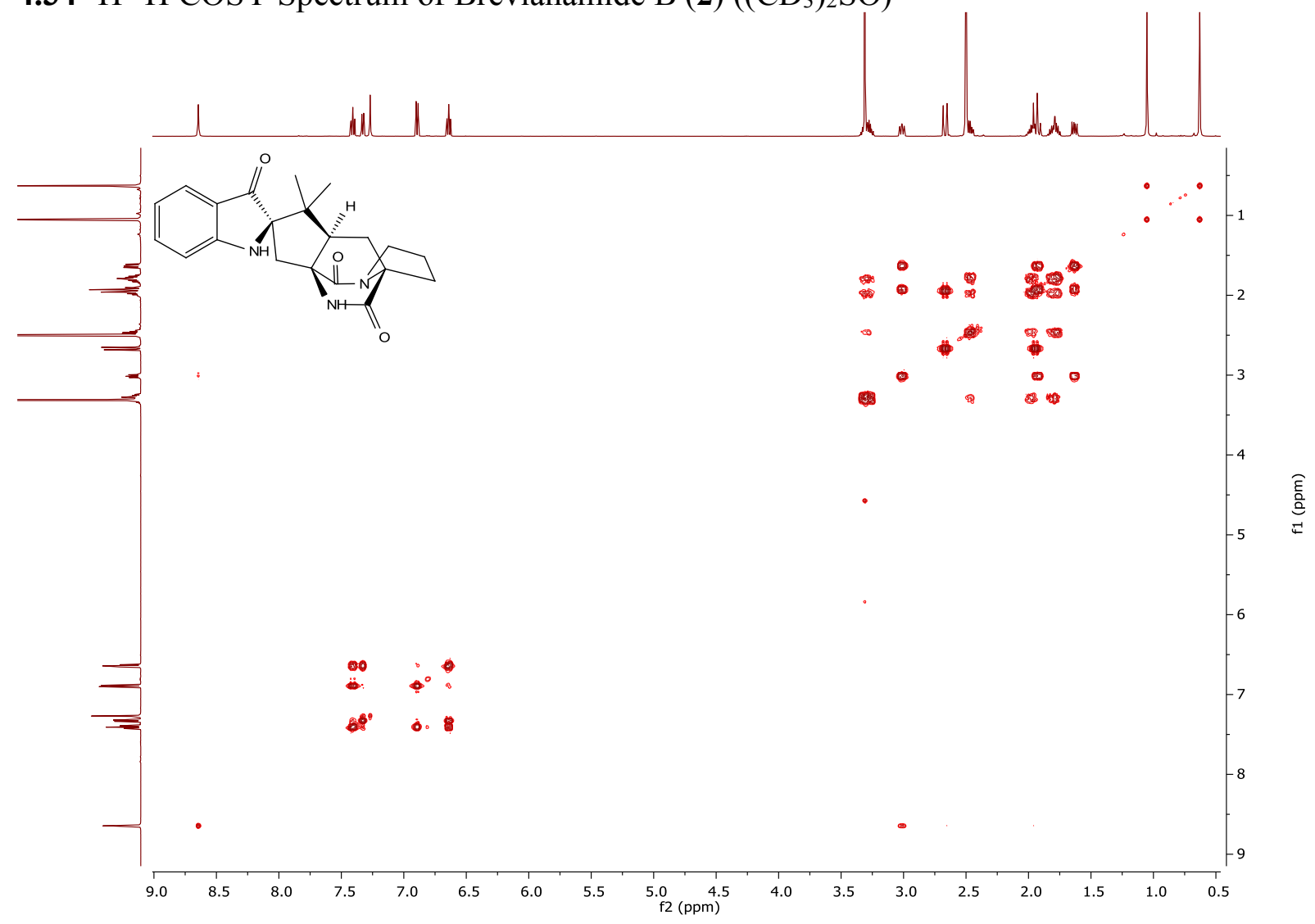

4.35 ${ }^{1} \mathrm{H}-{ }^{13} \mathrm{C}$ HSQC Spectrum of Brevianamide B (2) $\left(\left(\mathrm{CD}_{3}\right)_{2} \mathrm{SO}\right)$

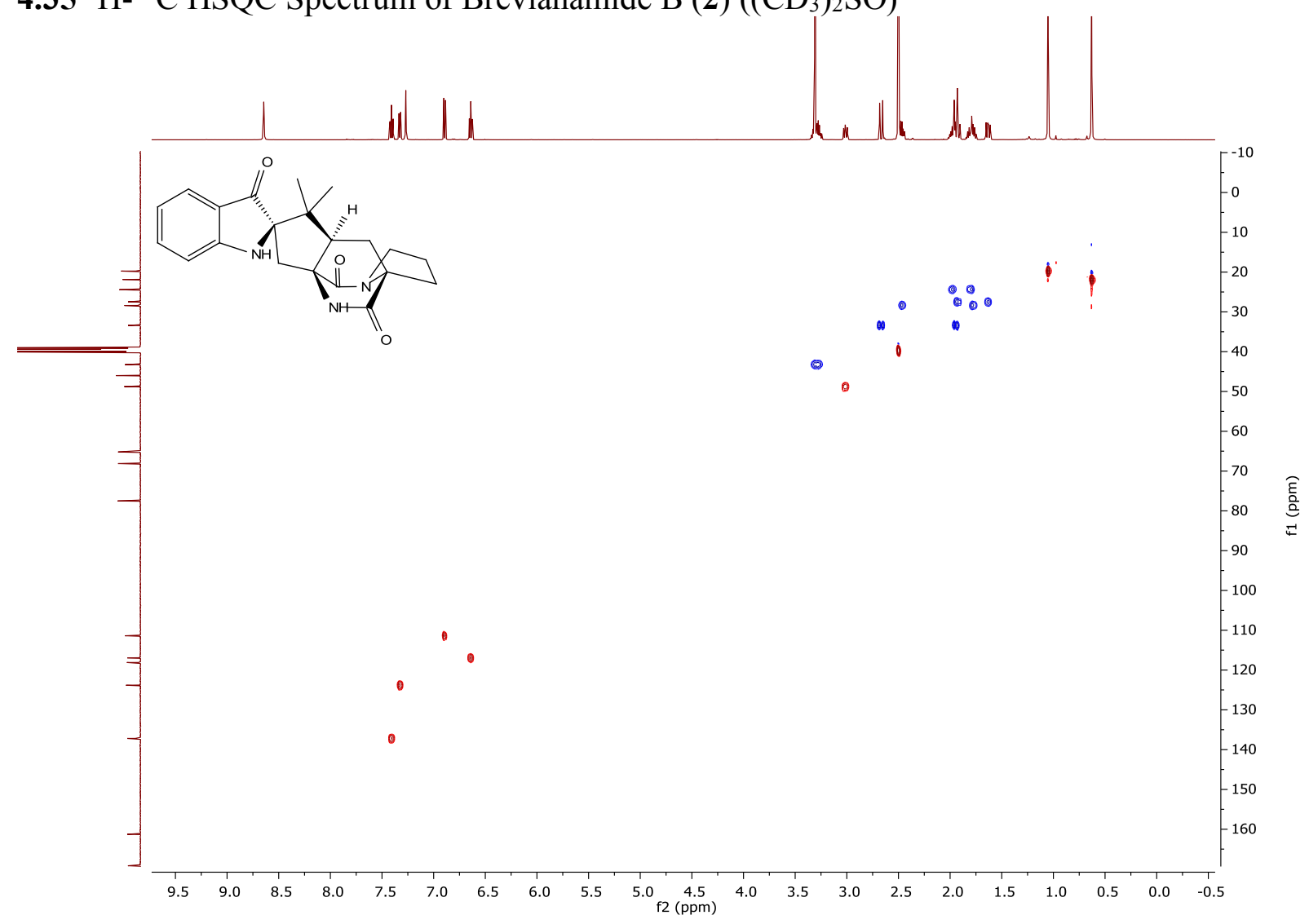


4.36 ${ }^{1} \mathrm{H}-{ }^{13} \mathrm{C}$ HMBC Spectrum of Brevianamide B (2) $\left(\left(\mathrm{CD}_{3}\right)_{2} \mathrm{SO}\right)$

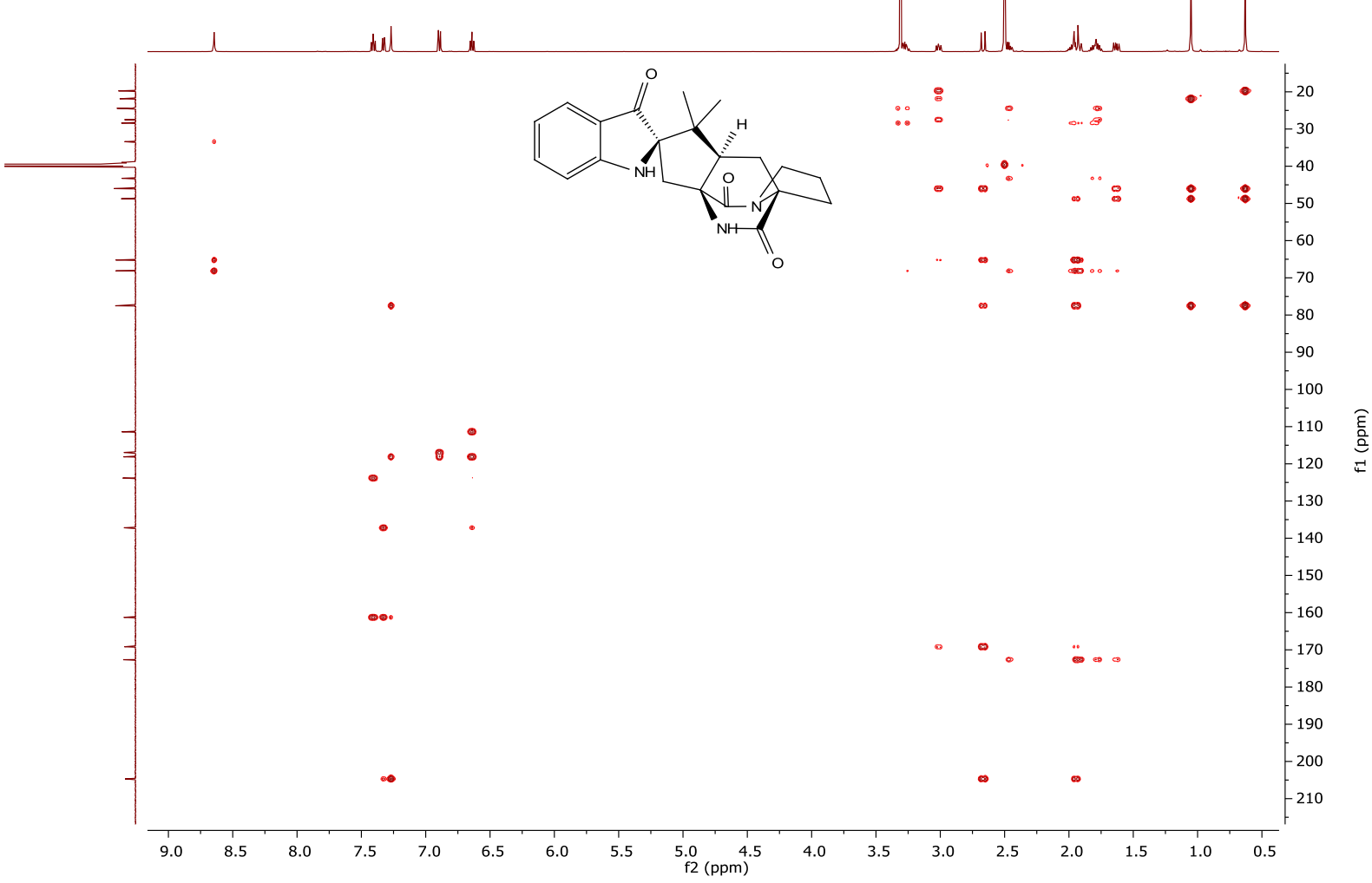

4.37 ${ }^{1} \mathrm{H}-{ }^{1} \mathrm{H}$ NOESY Spectrum of Brevianamide B (2) $\left(\left(\mathrm{CD}_{3}\right)_{2} \mathrm{SO}\right)$

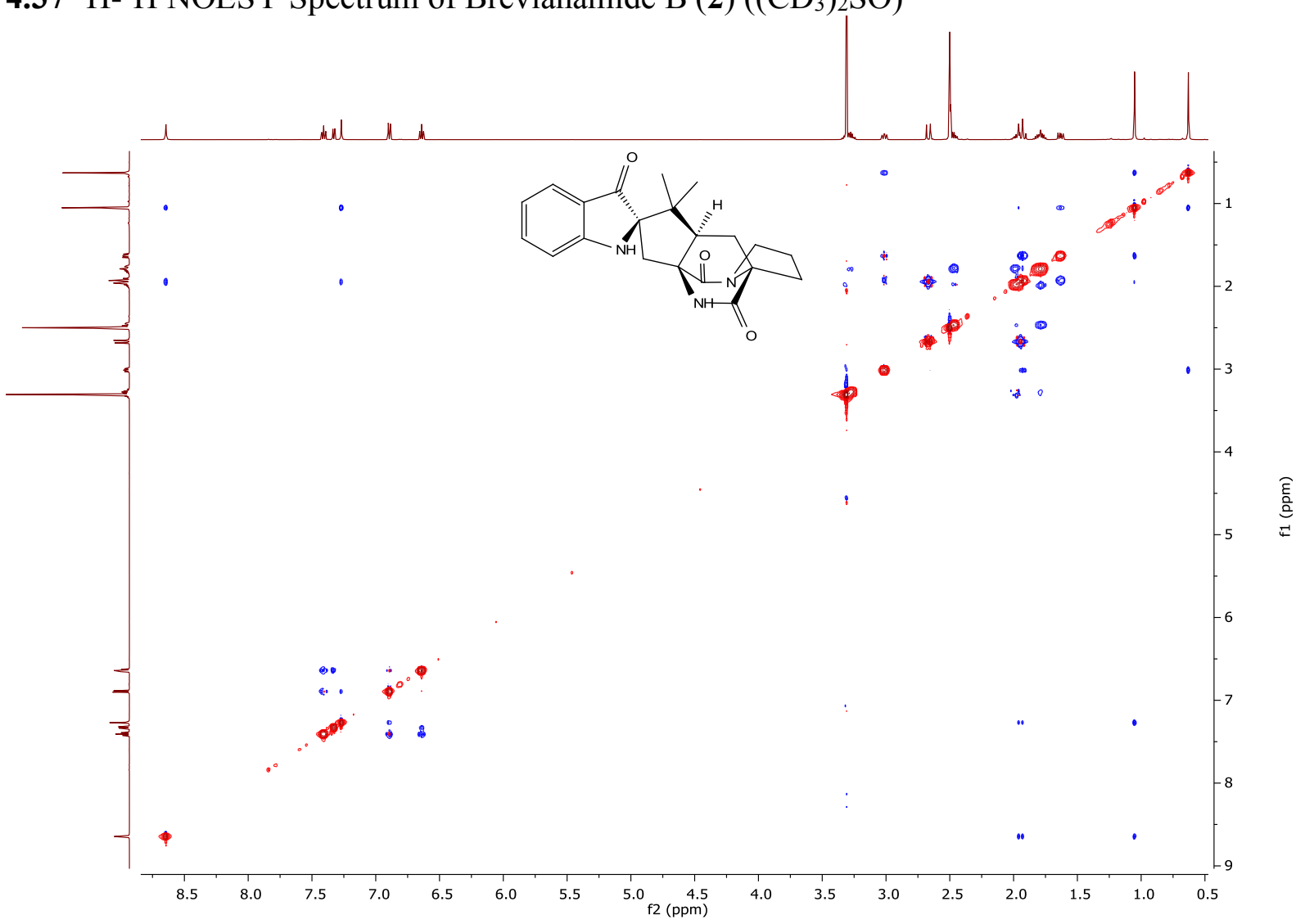

49 
4.38 ${ }^{1} \mathrm{H}$ NMR Spectrum of Compound $18\left(500 \mathrm{MHz}, \mathrm{CDCl}_{3}\right)$

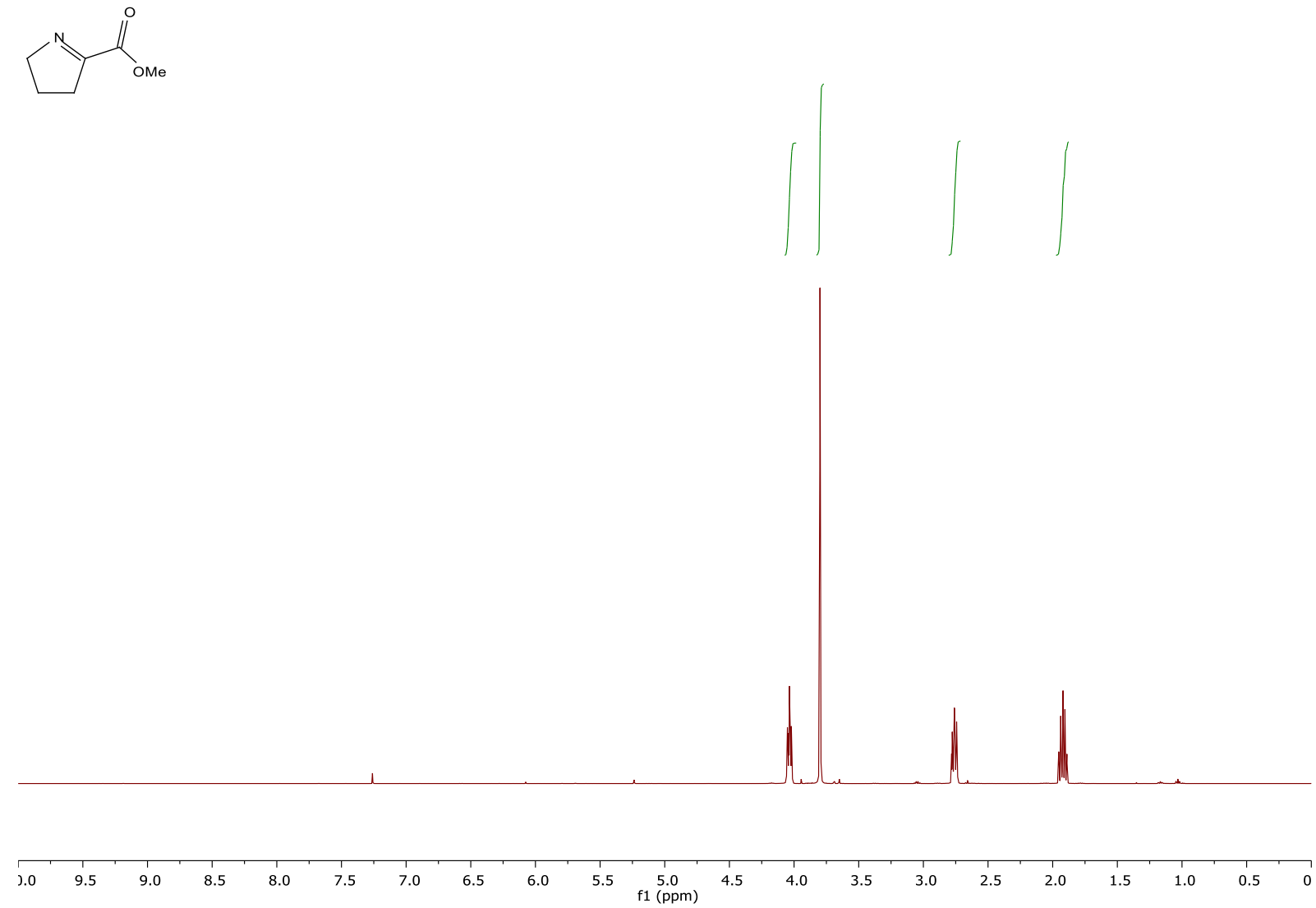

4.39 ${ }^{13} \mathrm{C}$ NMR Spectrum of Compound $18\left(126 \mathrm{MHz}, \mathrm{CDCl}_{3}\right)$

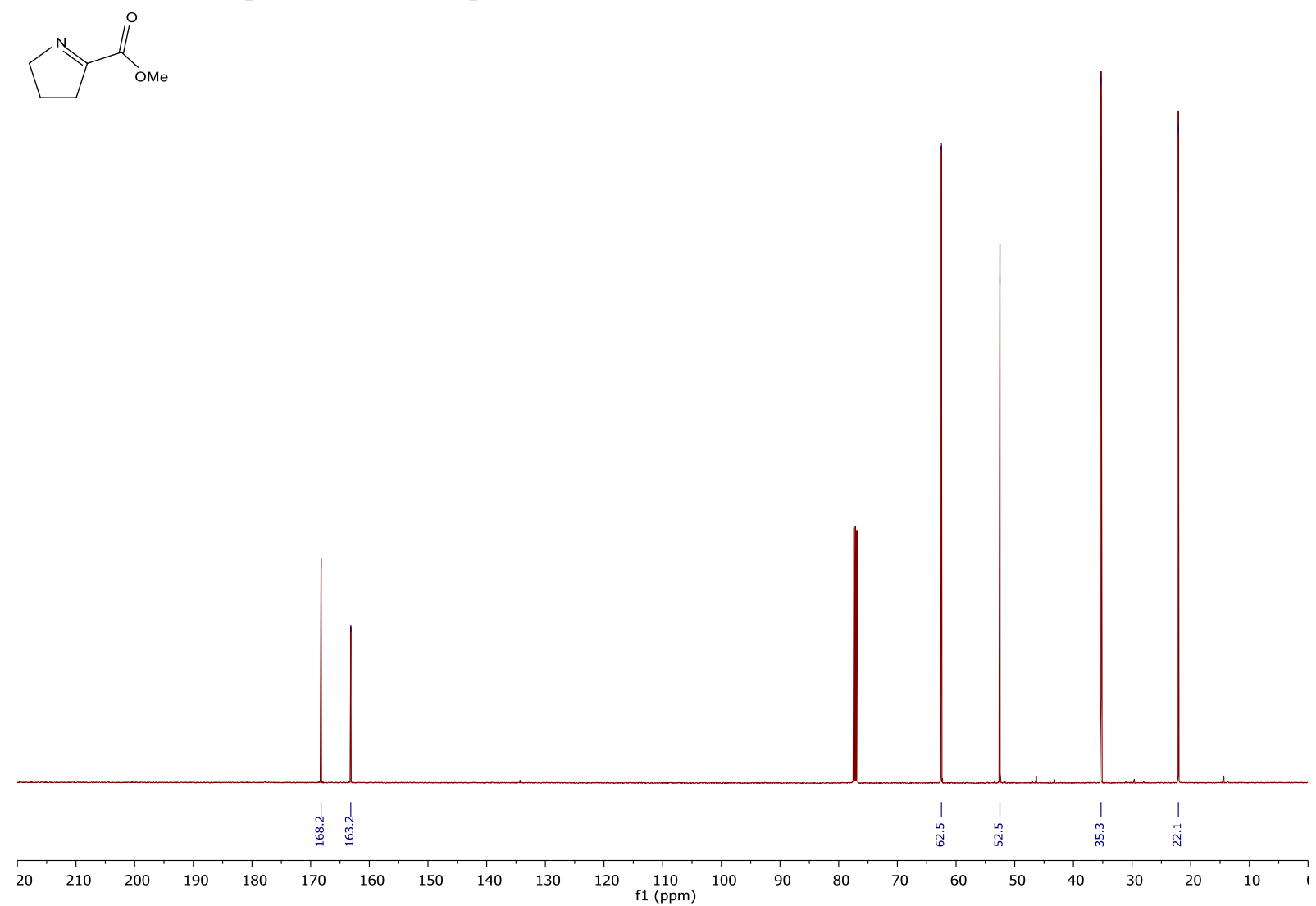


4.40 ${ }^{1} \mathrm{H}$ NMR Spectrum of 3-Chloro-3-methyl-1-butyne (500 MHz, $\mathrm{CDCl}_{3}$ )<smiles>CC1(C)C#CC1</smiles>

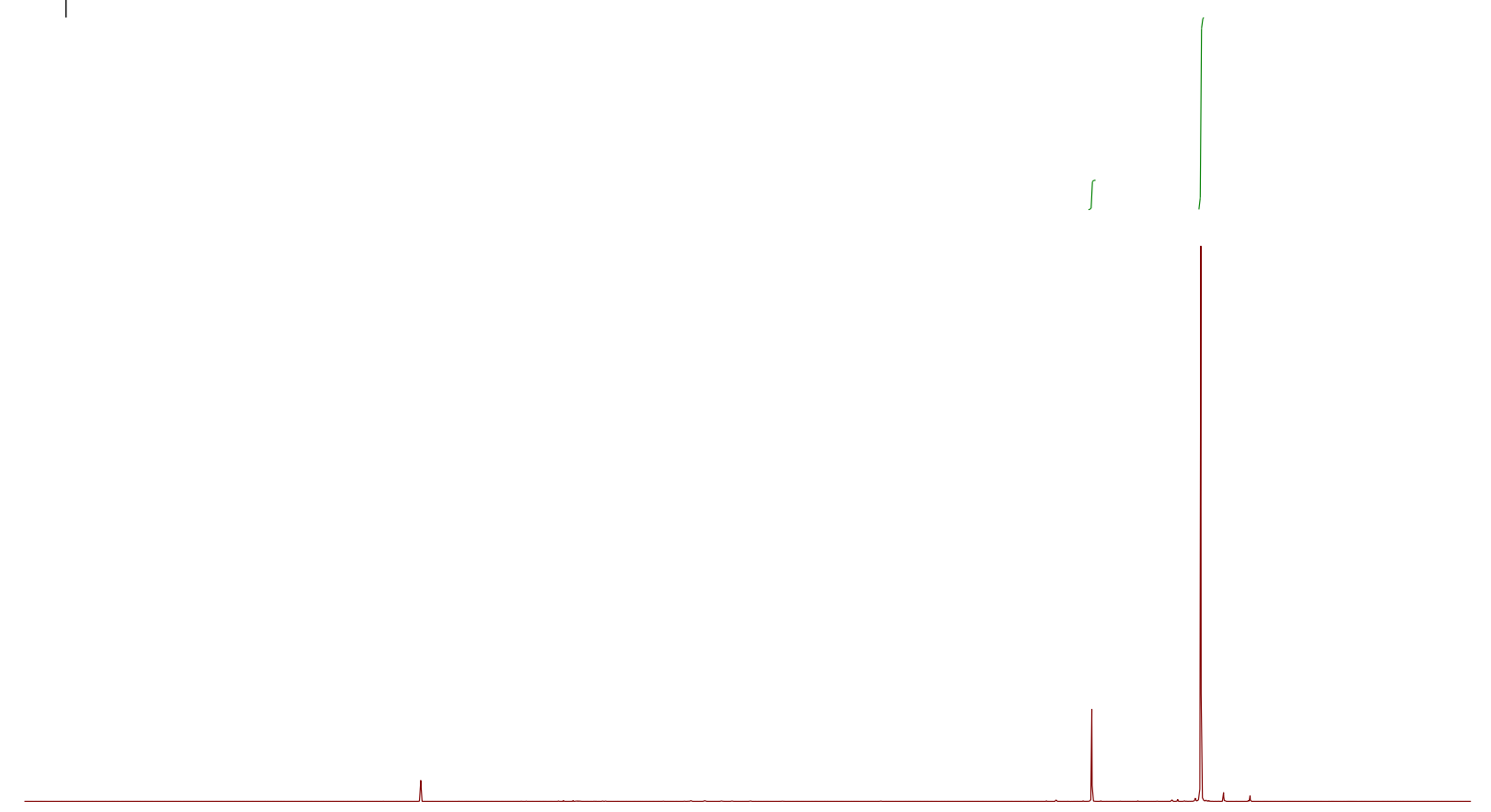

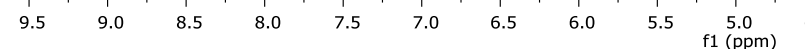

4.41 ${ }^{13} \mathrm{C}$ NMR Spectrum of 3-Chloro-3-methyl-1-butyne (101 $\mathrm{MHz}, \mathrm{CDCl}_{3}$ )
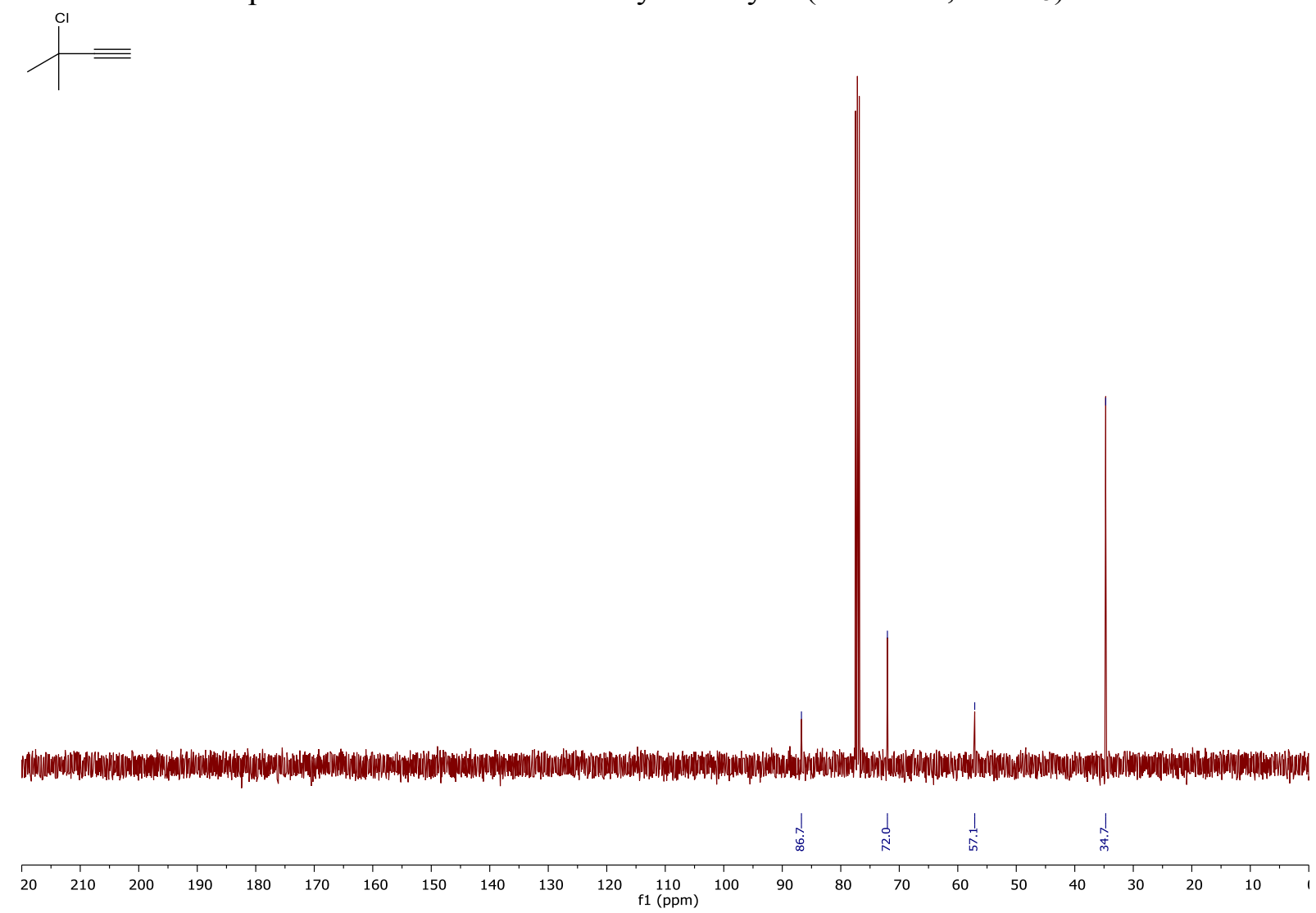
4.42 ${ }^{1} \mathrm{H}$ NMR Spectrum of 1,1-Dimethylallene (500 MHz, $\mathrm{CDCl}_{3}$ )
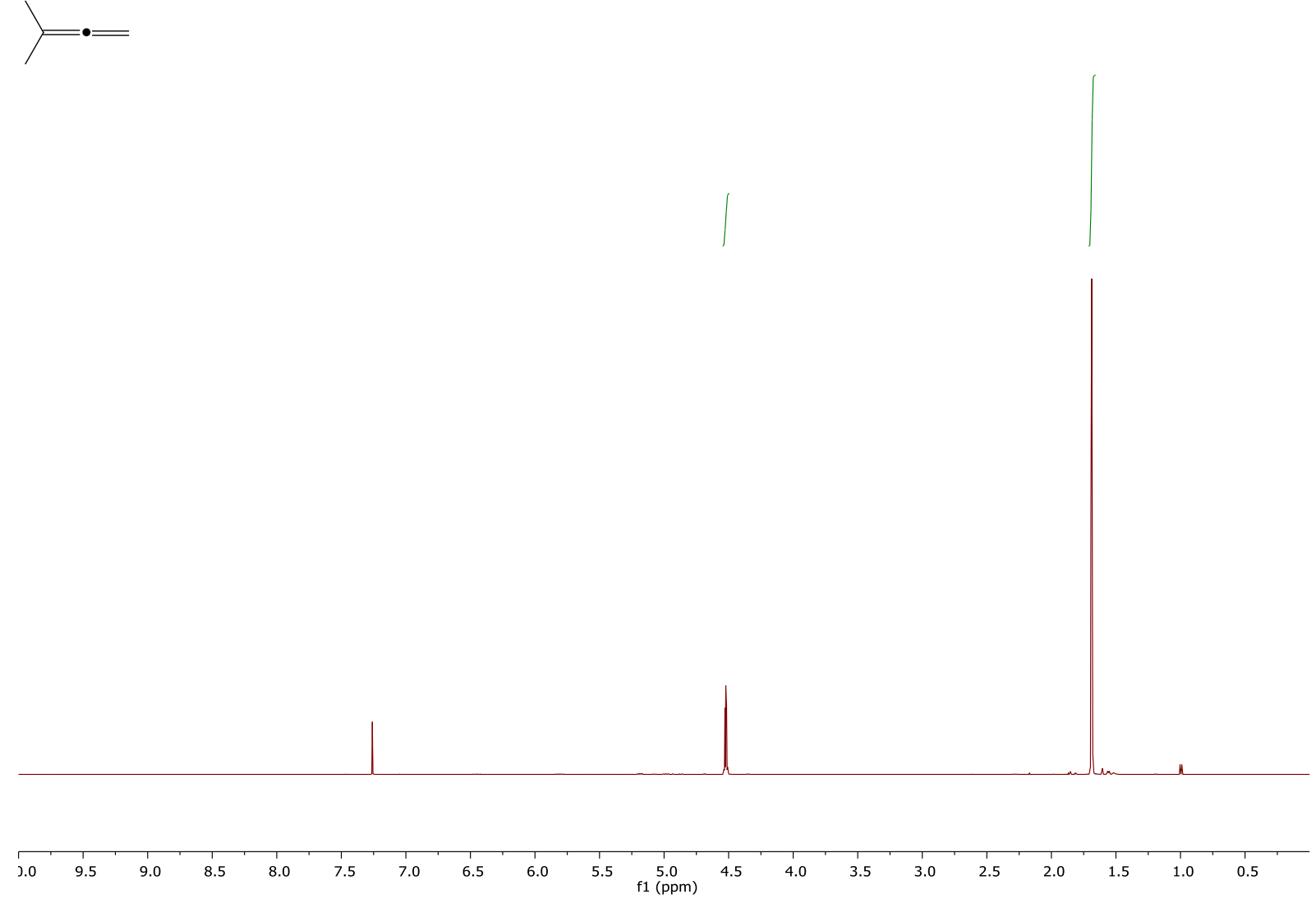

4.43 ${ }^{13} \mathrm{C}$ NMR Spectrum of 1,1-Dimethylallene (151 $\mathrm{MHz}, \mathrm{CDCl}_{3}$ )
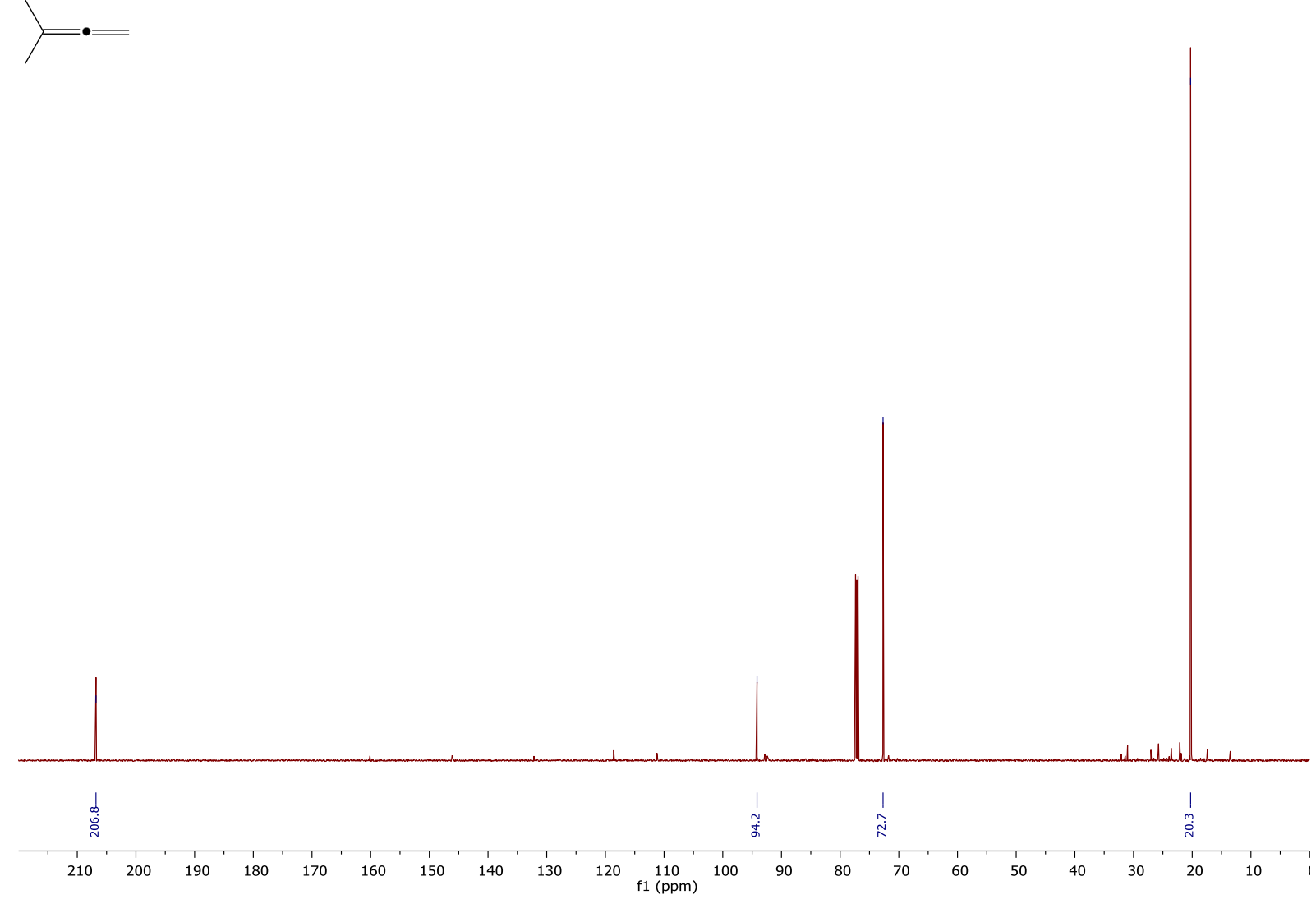
4.44 ${ }^{1} \mathrm{H}$ NMR Spectrum of $t$ - $\mathrm{BuOCl}\left(500 \mathrm{MHz}, \mathrm{CDCl}_{3}\right)$
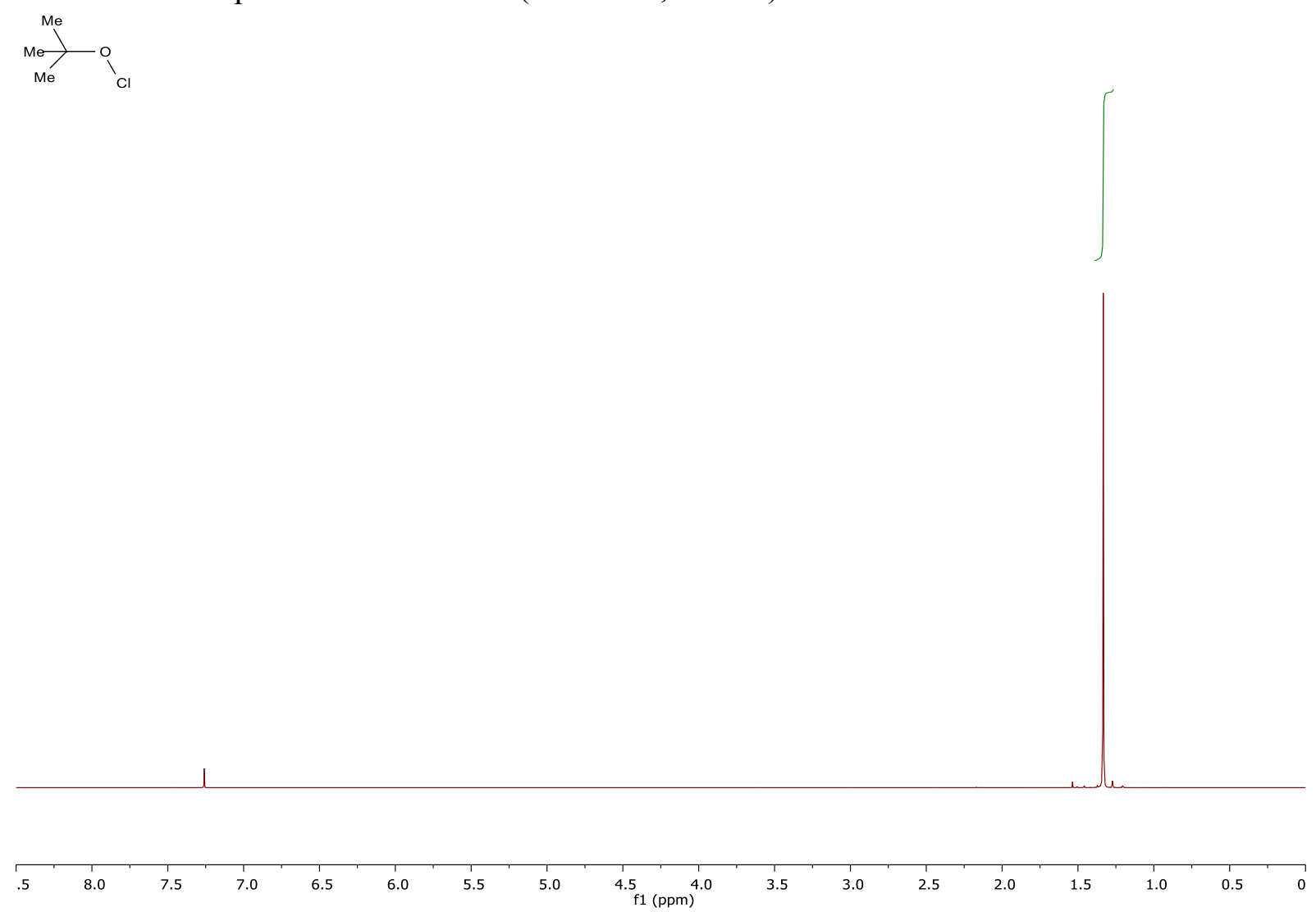

4.45 ${ }^{13} \mathrm{C}$ NMR Spectrum of $t$ - $\mathrm{BuOCl}\left(126 \mathrm{MHz}, \mathrm{CDCl}_{3}\right)$
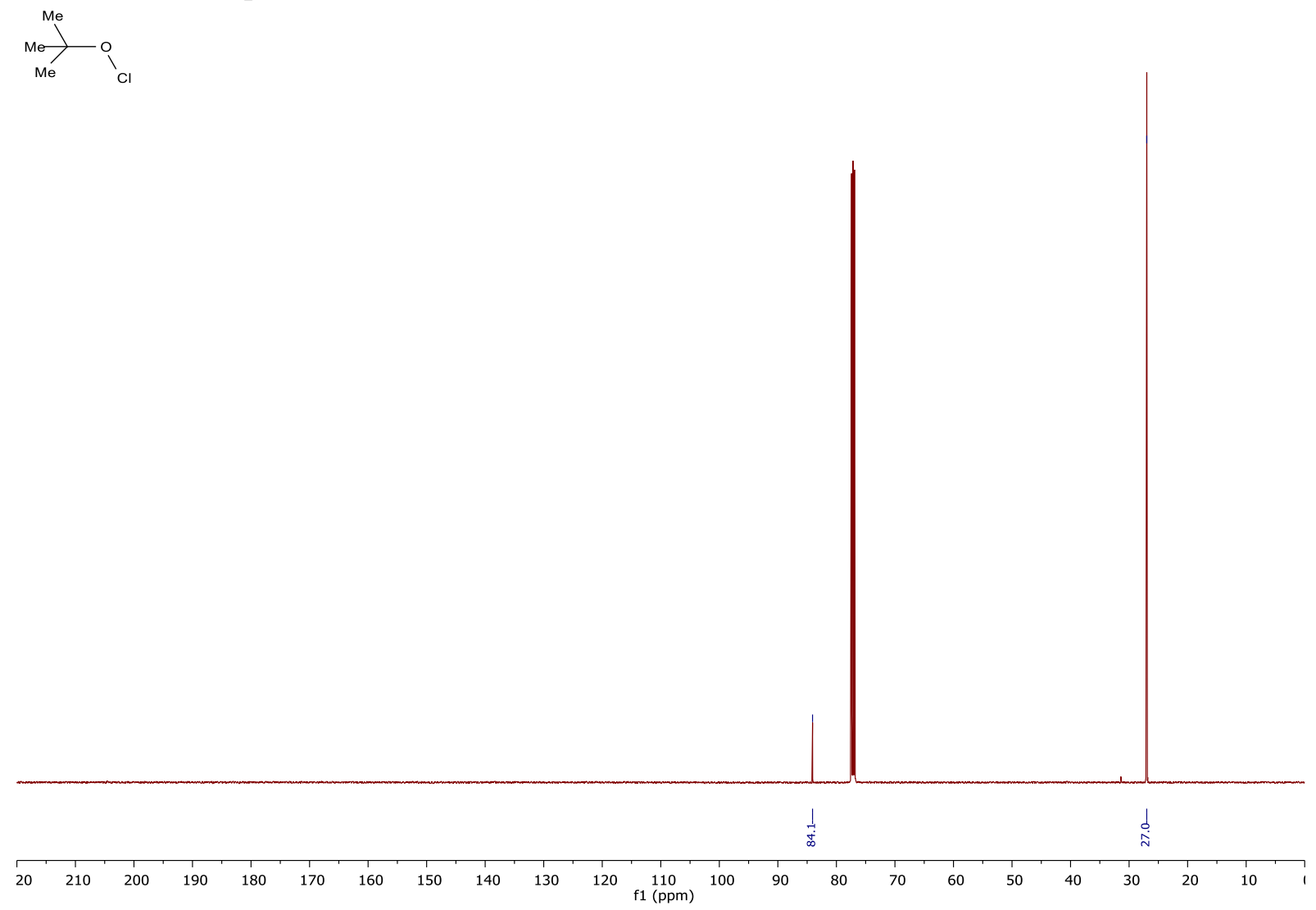

53 


\section{Chiral HPLC Chromatograms}

5.1 Chiral HPLC Chromatograms of Brevianamide A

$(+) /(-)$-brevianamide A (1)

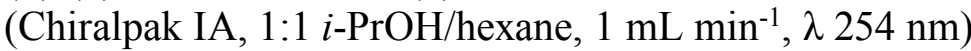

$\mathrm{t}_{\mathrm{R} 1}=5.40 \mathrm{~min}, \mathrm{t}_{\mathrm{R} 2}=6.74 \mathrm{~min}$.

\section{$<$ Chromatogram $>$}

$\mathrm{mV}$

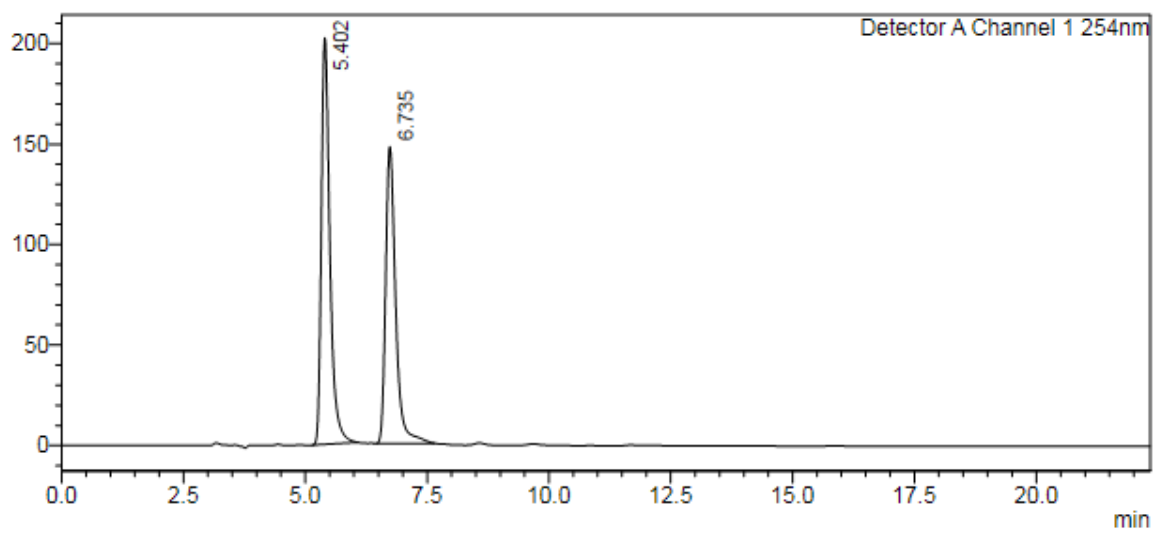

<Peak Table>

Detector A Channel $1254 \mathrm{~nm}$

\begin{tabular}{|c|c|c|c|c|c|c|c|}
\hline $\begin{array}{r}\text { Peak\# } \\
1\end{array}$ & $\begin{array}{r}\text { Ret. Time } \\
5.402\end{array}$ & $\begin{array}{l}\text { Area } \\
2456350\end{array}$ & $\begin{array}{l}\text { Height } \\
202003\end{array}$ & $\begin{array}{l}\text { Conc. } \\
53.925\end{array}$ & Unit & Mark & Name \\
\hline $\begin{array}{r}2 \\
\text { Total }\end{array}$ & 6.735 & $\begin{array}{l}2098773 \\
4555123\end{array}$ & $\begin{array}{l}147492 \\
349495\end{array}$ & 46.075 & & & \\
\hline
\end{tabular}

(+)-brevianamide A (1) e.r. 93:7

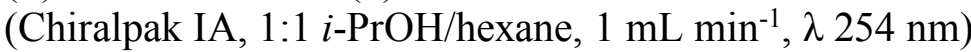

$t_{\text {Rminor }}=5.41 \mathrm{~min}, t_{\text {Rmajor }}=6.72 \mathrm{~min}$.

$<$ Chromatogram $>$

$\mathrm{mV}$

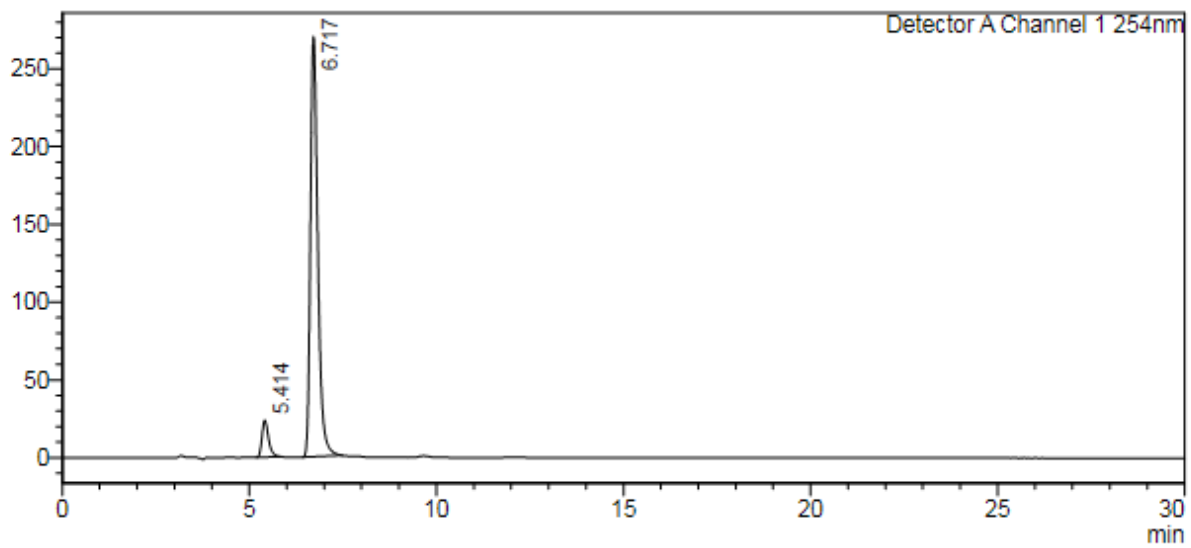

<Peak Table>

Detector A Channel $1254 \mathrm{~nm}$

\begin{tabular}{|c|c|c|c|c|c|c|c|}
\hline $\begin{array}{r}\text { Peak\# } \\
1\end{array}$ & $\begin{array}{r}\text { Ret. Time } \\
5.414\end{array}$ & $\begin{array}{l}\text { Area } \\
289622\end{array}$ & $\begin{array}{l}\text { Height } \\
23737\end{array}$ & $\begin{array}{l}\text { Conc. } \\
7.232\end{array}$ & Unit & Mark & Name \\
\hline 2 & 6.717 & 3715018 & 269840 & 92.768 & & & \\
\hline Total & & 4004641 & 293577 & & & & \\
\hline
\end{tabular}


(+)-brevianamide A (1) e.r. 99:1 after crystallisation

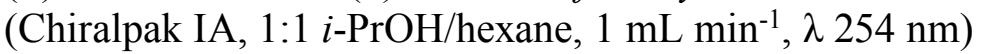

$\mathrm{t}_{\text {Rminor }}=5.51 \mathrm{~min}, \mathrm{t}_{\text {Rmajor }}=6.84 \mathrm{~min}$.

<Chromatogram>

$\mathrm{mV}$

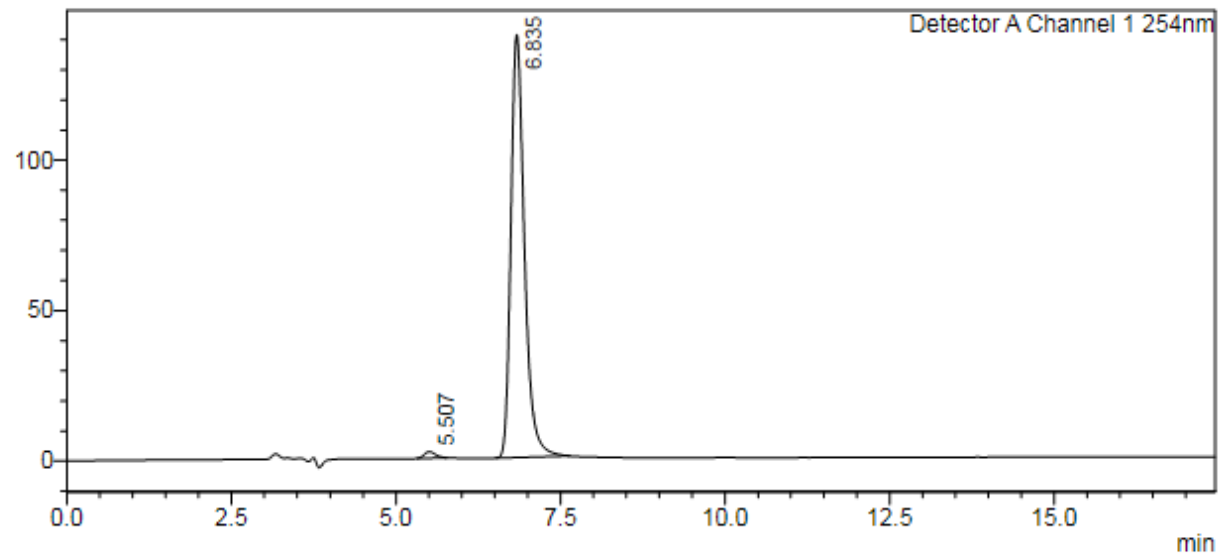

<Peak Table>

\begin{tabular}{|c|c|c|c|c|c|c|c|}
\hline $\begin{array}{c}\text { Detecto } \\
\text { Peak\# } \\
1\end{array}$ & $\begin{array}{r}\text { o A Channe } \\
\text { Ret. Time } \\
5.507\end{array}$ & $\begin{array}{l}254 \mathrm{~nm} \\
\text { Area } \\
24842\end{array}$ & $\begin{array}{l}\text { Height } \\
2113\end{array}$ & $\begin{array}{l}\text { Conc. } \\
1.220\end{array}$ & Unit & Mark & Name \\
\hline $\begin{array}{r}2 \\
\text { Total }\end{array}$ & 6.835 & $\begin{array}{l}2010703 \\
2035545\end{array}$ & $\begin{array}{l}140355 \\
142469\end{array}$ & 98.780 & & & \\
\hline
\end{tabular}

(-)-brevianamide A (1) e.r. 95:5

(Chiralpak IA, 1:1 $i$-PrOH/hexane, $1 \mathrm{~mL} \mathrm{~min}^{-1}, \lambda 254 \mathrm{~nm}$ )

$t_{\text {Rmajor }}=5.38 \mathrm{~min}, \mathrm{t}_{\mathrm{Rminor}}=6.74 \mathrm{~min}$.

\section{<Chromatogram>}

$\mathrm{mV}$

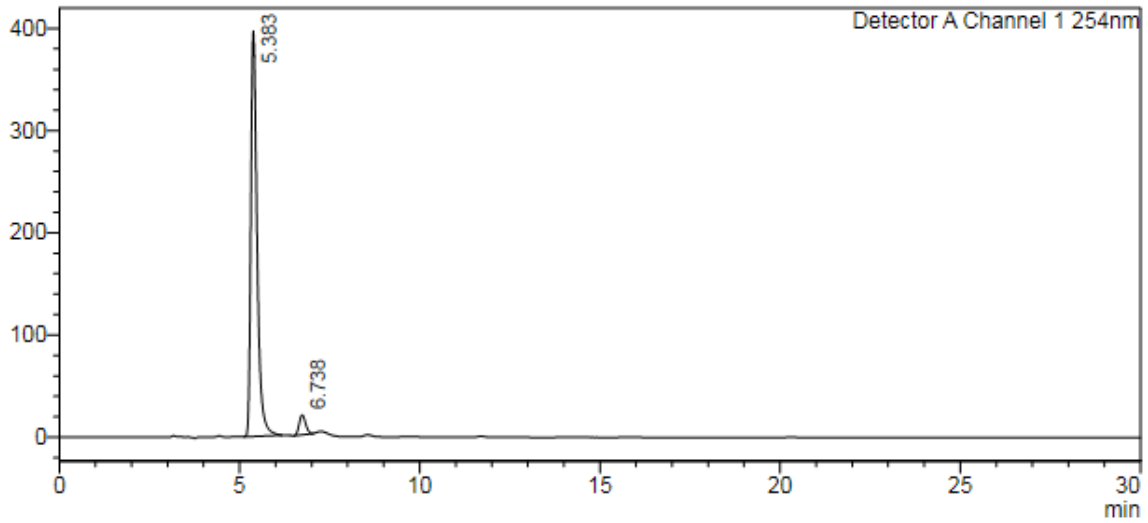

\section{<Peak Table>}

Detector A Channel $1254 \mathrm{~nm}$

Peak\# Ret. Time Area

\begin{tabular}{|r|r|r|r|r|r|r|r}
1 & 5.383 & 4758681 & 396107 & Conc. & Unit & Mark & Name \\
\hline 2 & 6.738 & 241511 & 19400 & 4.830 & & & \\
Total & & 5000192 & 415507 & & & & \\
\hline
\end{tabular}


5.2 Chiral HPLC Chromatograms of Brevianamide B

$(+) /(-)$-Brevianamide B (2)

(Chiralpak IA, 1:3 EtOH/hexane, $1 \mathrm{~mL} \min ^{-1}, \lambda 254 \mathrm{~nm}$ )

$\mathrm{t}_{\mathrm{R} 1}=13.86 \mathrm{~min}, \mathrm{t}_{\mathrm{R} 2}=26.03 \mathrm{~min}$.

\section{$<$ Chromatogram>}

$\mathrm{mV}$

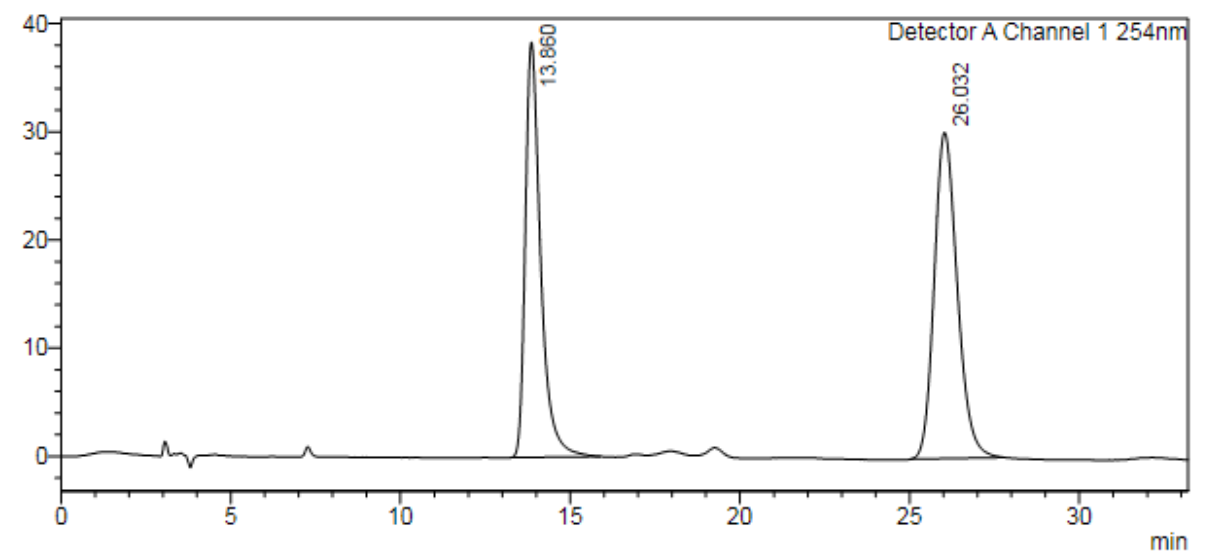

<Peak Table>

Detector A Channel $1254 \mathrm{~nm}$

\begin{tabular}{|c|c|c|c|c|c|c|c|}
\hline $\begin{array}{r}\text { Peak\# } \\
1\end{array}$ & $\begin{array}{r}\text { Ret. Time } \\
13.860\end{array}$ & $\begin{array}{l}\text { Area } \\
1202069\end{array}$ & $\begin{array}{l}\text { Height } \\
38310\end{array}$ & $\begin{array}{l}\text { Conc. } \\
45.782\end{array}$ & Unit & Mark & Name \\
\hline $\begin{array}{r}2 \\
\text { Total }\end{array}$ & 26.032 & $\begin{array}{l}1423551 \\
2625620\end{array}$ & $\begin{array}{l}30114 \\
68424\end{array}$ & 54.218 & & & \\
\hline
\end{tabular}

(+)-Brevianamide B (2) e.r. 93:7

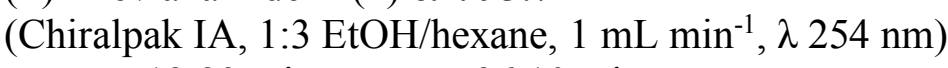

$t_{\text {Rminor }}=13.99 \mathrm{~min}, t_{\text {Rmajor }}=26.10 \mathrm{~min}$.

\section{$<$ Chromatogram>}

$\mathrm{mV}$

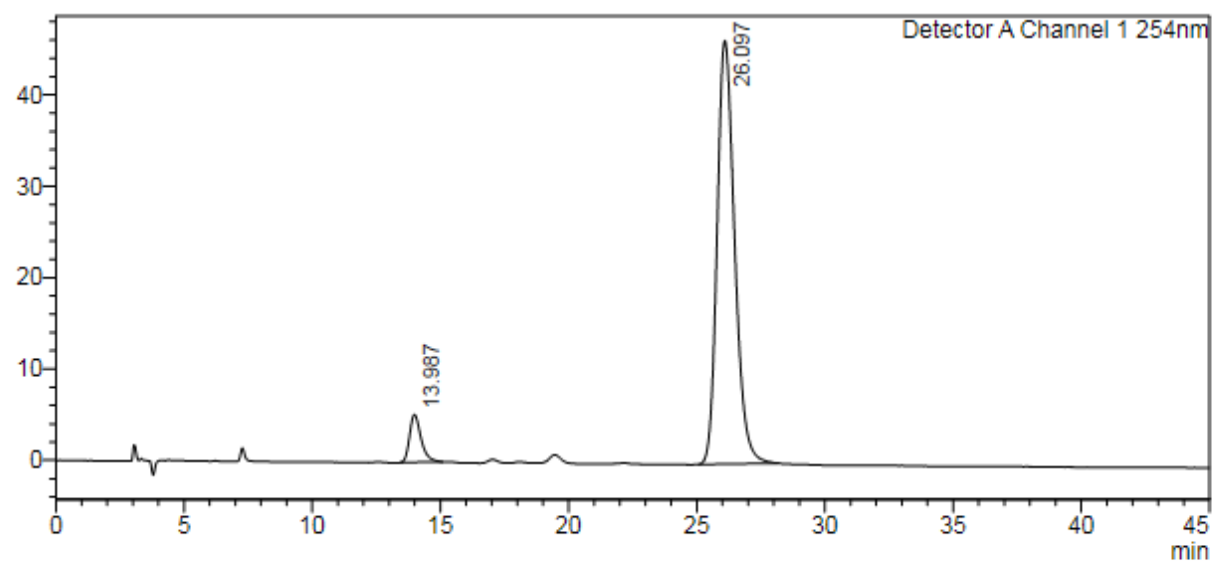

<Peak Table>

Detector A Channel $1254 \mathrm{~nm}$

Peak\# Ret. Time Area

\begin{tabular}{r|r|r|r|r|r|r|r} 
Peak\# Ret. Time & Area & Height & Conc. & Unit & Mark & Name \\
\hline 1 & 13.987 & 162378 & 5219 & 6.875 & & & \\
\hline 2 & 26.097 & 2199609 & 46337 & 93.125 & & & \\
Total & & 2361987 & 51556 & & & & \\
\hline
\end{tabular}


(-)-Brevianamide B (2) e.r. 92:8

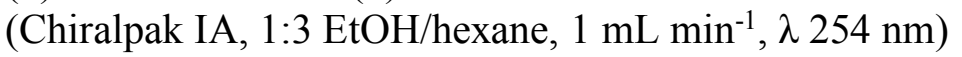

$t_{\text {Rmajor }}=13.86 \mathrm{~min}, t_{\text {Rminor }}=26.14 \mathrm{~min}$.

\section{$<$ Chromatogram $>$}

$\mathrm{mV}$

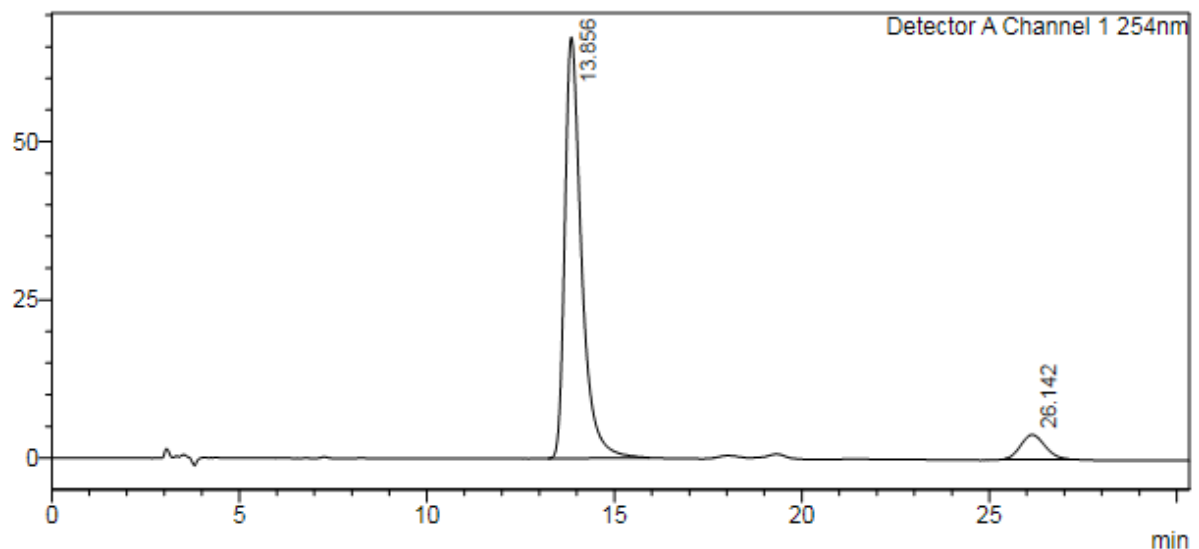

<Peak Table>

Detector A Channel $1254 \mathrm{~nm}$
Peak\# Ret. Time

\begin{tabular}{r|r|r|}
1 & 13.856 & 2058078 \\
\hline 2 & 26.142 & 180065 \\
\hline
\end{tabular}

\begin{tabular}{l|l|l|l} 
Height & Conc. & Unit & Mark
\end{tabular}

Name

\begin{tabular}{lr|r|} 
Total & 2238144 \\
\hline
\end{tabular}

3929

70484 


\section{Crystal Data and Experimental}

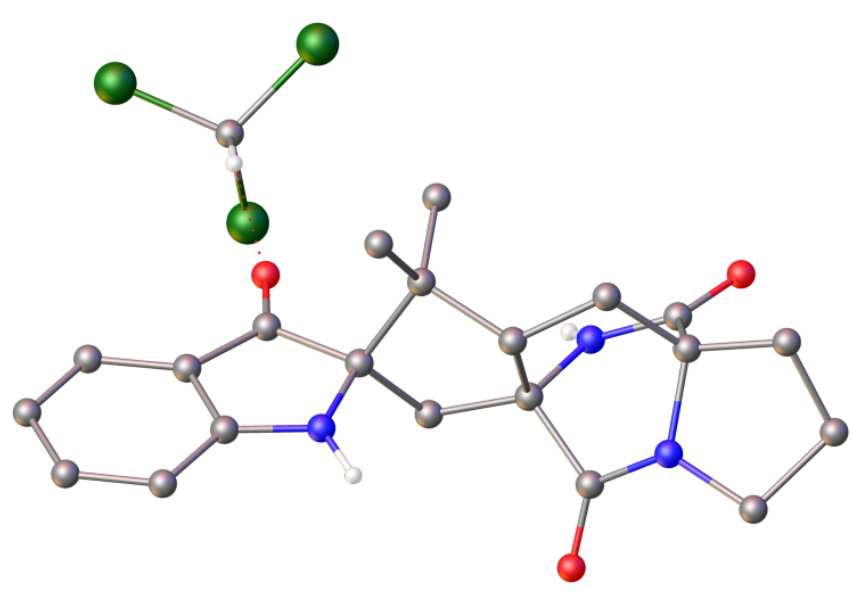

Experimental. Single yellow plate-shaped crystals of AL19001 were recrystallised from a mixture of chloroform and diethyl ether by vapour diffusion. A suitable crystal $0.24 \times 0.09 \times 0.05 \mathrm{~mm}^{3}$ was selected and mounted on a MITIGEN holder in Paratone oil on an Rigaku Oxford Diffraction SuperNova diffractometer. The crystal was kept at a steady $T=120.0 \mathrm{~K}$ during data collection. The structure was solved with the ShelXT (Sheldrick, 2015) structure solution program using the Intrinsic Phasing solution method and by using 0lex2 (Dolomanov et al., 2009 ) as the graphical interface. The model was refined with version 2018/3 of ShelXL (Sheldrick, 2015) using Least Squares minimisation.

Crystal Data. $\mathrm{C}_{22} \mathrm{H}_{24} \mathrm{Cl}_{3} \mathrm{~N}_{3} \mathrm{O}_{3}, M_{r}=484.79$, monoclinic, $I 2$ (No. 5), $a=13.2441(2) \AA, \quad b=8.65980(10) \AA, \quad c=$ 20.3280(2) $\AA, \quad \beta=104.2140(10)^{\circ}, \quad \alpha=\gamma=90^{\circ}, \quad V=$ $2260.07(5) \AA^{3}, T=120.0 \mathrm{~K}, Z=4, Z^{\prime}=1, \mu\left(\mathrm{CuK}_{\alpha}\right)=3.919$, 22429 reflections measured, 4696 unique $\left(R_{\text {int }}=0.0715\right)$ which were used in all calculations. The final $w R_{2}$ was 0.1135 (all data) and $R_{1}$ was 0.0434 (I > 2(I)).

\begin{tabular}{|c|c|}
\hline Compound & AL19001 \\
\hline Formula & $\mathrm{C}_{22} \mathrm{H}_{24} \mathrm{Cl}_{3} \mathrm{~N}_{3} \mathrm{O}_{3}$ \\
\hline$D_{\text {calc. }} / \mathrm{g} \mathrm{cm}^{-3}$ & 1.425 \\
\hline$\mu / \mathrm{mm}^{-1}$ & 3.919 \\
\hline Formula Weight & 484.79 \\
\hline Colour & yellow \\
\hline Shape & plate \\
\hline Size $/ \mathrm{mm}^{3}$ & $0.24 \times 0.09 \times 0.05$ \\
\hline$T / \mathrm{K}$ & 120.0 \\
\hline Crystal System & monoclinic \\
\hline Flack Parameter & $-0.007(8)$ \\
\hline Hooft Parameter & $-0.004(5)$ \\
\hline Space Group & $I 2$ \\
\hline$a / \AA ̊$ & $13.2441(2)$ \\
\hline$b / \AA ̊$ & $8.65980(10)$ \\
\hline$c / \AA ̊$ & $20.3280(2)$ \\
\hline$\left.\alpha\right|^{\circ}$ & 90 \\
\hline$\beta /^{\circ}$ & $104.2140(10)$ \\
\hline$\gamma /^{\circ}$ & 90 \\
\hline $\mathrm{V} / \AA^{3}$ & $2260.07(5)$ \\
\hline$Z$ & 4 \\
\hline$Z^{\prime}$ & 1 \\
\hline Wavelength/Å & 1.54184 \\
\hline Radiation type & $\mathrm{CuK}_{\alpha}$ \\
\hline$\Theta_{\min } /^{\circ}$ & 3.618 \\
\hline$\Theta_{\max } /^{\circ}$ & 76.076 \\
\hline Measured Refl. & 22429 \\
\hline Independent Refl. & 4696 \\
\hline $\begin{array}{l}\text { Reflections with I > } \\
\text { 2(I) }\end{array}$ & 4622 \\
\hline$R_{\text {int }}$ & 0.0715 \\
\hline Parameters & 301 \\
\hline Restraints & 12 \\
\hline Largest Peak & 0.283 \\
\hline Deepest Hole & -0.377 \\
\hline GooF & 1.040 \\
\hline$w R_{2}$ (all data) & 0.1135 \\
\hline$w R_{2}$ & 0.1130 \\
\hline$R_{1}$ (all data) & 0.0437 \\
\hline
\end{tabular}




\section{Structure Quality Indicators}

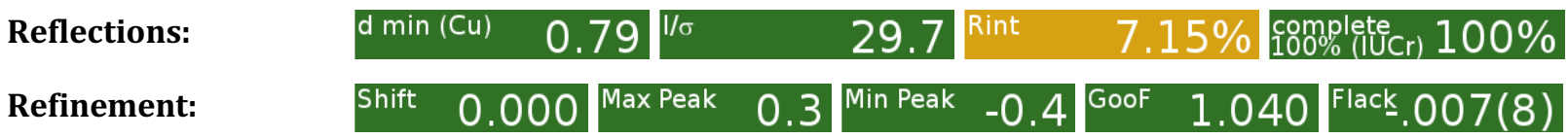

A yellow plate-shaped crystal with dimensions $0.24 \times 0.09 \times 0.05 \mathrm{~mm}^{3}$ was mounted on a MITIGEN holder in Paratone oil. Data were collected using an Rigaku Oxford Diffraction SuperNova diffractometer equipped with an Oxford Cryosystems Cryostream 700+ low-temperature device operating at $T=120.0 \mathrm{~K}$.

Data were measured using $\omega$ scans using $\mathrm{CuK}_{\alpha}$ radiation. The total number of runs and images was based on the strategy calculation from the program CrysAlisPro (Rigaku, V1.171.39.46, 2018) The maximum resolution that was achieved was $\Theta=76.076^{\circ}(0.79 \AA)$.

The diffraction pattern was indexed The total number of runs and images was based on the strategy calculation from the program CrysAlisPro (Rigaku, V1.171.39.46, 2018) and the unit cell was refined using CrysAlisPro (Rigaku, V1.171.39.46, 2018) on 18841 reflections, $84 \%$ of the observed reflections.

Data reduction, scaling and absorption corrections were performed using CrysAlisPro (Rigaku, V1.171.39.46, 2018). The final completeness is $100.00 \%$ out to $76.076^{\circ}$ in $\Theta$. A multi-scan absorption correction was performed using CrysAlisPro 1.171.39.46 (Rigaku Oxford Diffraction, 2018) using spherical harmonicsas implemented in SCALE3 ABSPACK.. The absorption coefficient $\mu$ of this material is $3.919 \mathrm{~mm}^{-1}$ at this wavelength $(\lambda=1.542 \AA)$ and the minimum and maximum transmissions are 0.732 and 1.000 .

The structure was solved and the space group I2 (\# 5) determined by the ShelXT (Sheldrick, 2015) structure solution program using Intrinsic Phasing and refined by Least Squares using version 2018/3 of ShelXL (Sheldrick, 2015). All non-hydrogen atoms were refined anisotropically. Hydrogen atom positions were calculated geometrically and refined using the riding model. Most hydrogen atom positions were calculated geometrically and refined using the riding model, but some hydrogen atoms were refined freely.

_refine_special_details: $\mathrm{N}$-bound $\mathrm{H}$ atoms were identified from a difference Fourier map and freely refined. C14 was modelled as disordered over two sites consistent with a residual peak in a difference map. Geometric and displacement ellipsoid restraints were used.

The Flack parameter was refined to -0.007(8). Determination of absolute structure using Bayesian statistics on Bijvoet differences using the Olex2 results in -0.004(5). Note: The Flack parameter is used to determine chirality of the crystal studied, the value should be near 0 , a value of 1 means that the stereochemistry is wrong and the model should be inverted. A value of 0.5 means that the crystal consists of a racemic mixture of the two enantiomers. 
